\title{
Nuclear Facility Accident (NFAC) Unit Test Report For HPAC Version 6.4
}

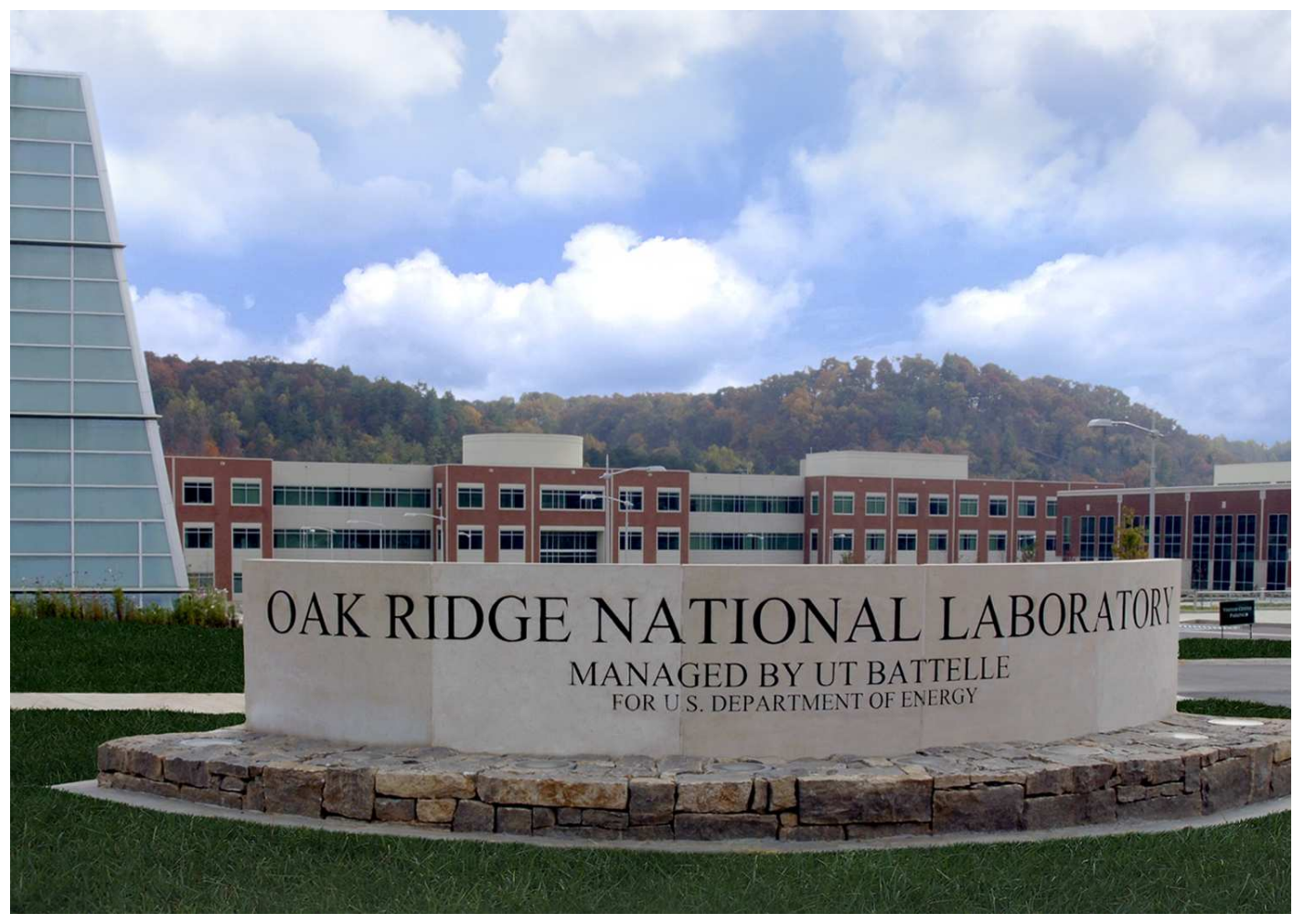

Ronald W. Lee

C. David Sulfredge

Approved for public release.

Distribution is unlimited.

March, 2017 


\section{DOCUMENT AVAILABILITY}

Reports produced after January 1, 1996, are generally available free via the U.S. Department of Energy (DOE) SciTech Connect.

Website: http://www.osti.gov/scitech

Reports produced before January 1, 1996, may be purchased by members of the public from the following source.

National Technical Information Service

5825 Port Royal Road

Springfield, VA 22161

Telephone: 703-605-6000 (1-800-553-6847)

TDD: 703-487-4639

Fax: 703-605-6900

E-mail: info@ntis.fedworld.gov

Website: http://www.ntis.gov/help/ordermethods.aspx

Reports are available to DOE employees, DOE contractors, Energy Technology Data Exchange (ETDE) representatives, and International Nuclear Information System (INIS) representatives from the following source.

Office of Scientific and Technical Information

P.O. Box 62

Oak Ridge, TN 37831

Telephone: 865-576-8401

Fax: 865-576-5728

E-mail: reports@osti.gov

Website: http://www.osti.gov/contact.html

This report was prepared as an account of work sponsored by an agency of the United States Government. Neither the United States Government nor any agency thereof, nor any of their employees, makes any warranty, express or implied, or assumes any legal liability or responsibility for the accuracy, completeness, or usefulness of any information, apparatus, product, or process disclosed, or represents that its use would not infringe privately owned rights. Reference herein to any specific commercial product, process, or service by trade name, trademark, manufacturer, or otherwise, does not necessarily constitute or imply its endorsement, recommendation, or favoring by the United States Government or any agency thereof. The views and opinions of authors expressed herein do not necessarily state or reflect those of the United States Government or any agency thereof. 
Computational Sciences and Engineering Division

\title{
NUCLEAR FACILITY ACCIDENT (NFAC) UNIT TEST REPORT FOR HPAC VERSION 6.4
}

\author{
Ronald W. Lee \\ C. David Sulfredge
}

Date Published: March, 2017

Prepared by OAK RIDGE NATIONAL LABORATORY

Oak Ridge, Tennessee 37831-6285

managed by

UT-BATTELLE, LLC

for the

U.S. DEPARTMENT OF ENERGY

under contract DE-AC05-00OR22725 



\section{CONTENTS}

LIST OF FIGURES $\ldots \ldots \ldots \ldots \ldots \ldots \ldots \ldots \ldots$

LIST OF TABLES $\ldots \ldots \ldots \ldots \ldots \ldots \ldots \ldots$ vii

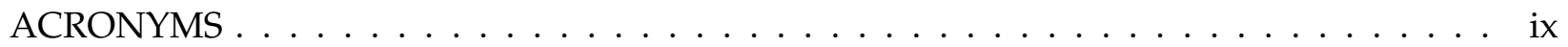

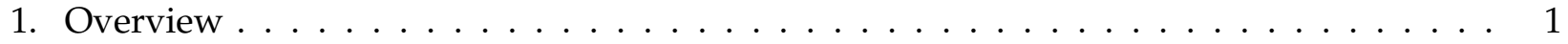

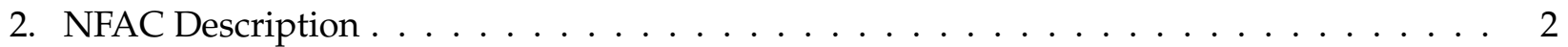

2.1 Source Models . . . . . . . . . . . . . . . . . . . . . . . . . . . . 2

2.2 Material Modes . . . . . . . . . . . . . . . . . . . 3

2.3 PIR Release Modes $\ldots \ldots \ldots \ldots \ldots$

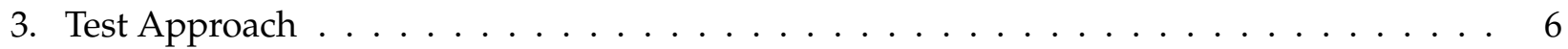

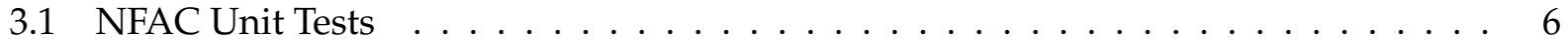

3.2 NFAC-RTH Integration Tests $\ldots \ldots \ldots \ldots \ldots \ldots$

3.3 NFAC-RTH-SCIPUFF Integration Tests $\ldots \ldots \ldots \ldots \ldots \ldots$

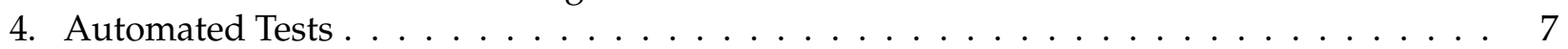

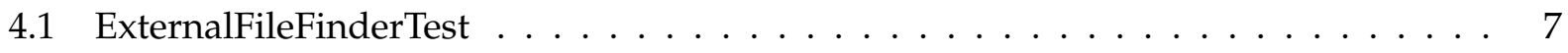

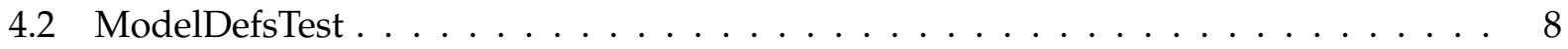

4.3 ModelTimesTest . . . . . . . . . . . . . . . . . . . . . . 22

4.4 PIRModelDefsTest . . . . . . . . . . . . . . . . . . . . . . 24

4.5 ParticleGroupsMgrTest . . . . . . . . . . . . . . . . . . . . . . . 34

4.6 SourceTermTableTest . . . . . . . . . . . . . . . . . . 35

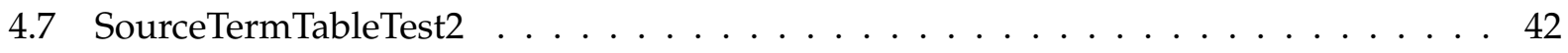

4.8 RTHTimesTest . . . . . . . . . . . . . . . . . . . . . . . . . . . . 42

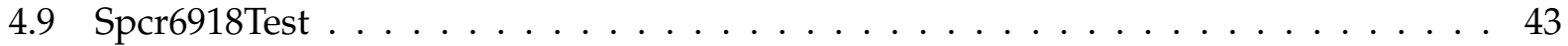

4.9 .1 No Shutdown Full . . . . . . . . . . . . . . . . . . . . . 43

4.9 .2 No Shutdown Partial . . . . . . . . . . . . . . . . . . . . . . . . . . . . . . . . . .

4.9 .3 Shutdown Full . . . . . . . . . . . . . . . . . . . . . . . . . . . . . . . . . . . . 44

4.9 .4 Shutdown Partial . . . . . . . . . . . . . . . . . . . . . . 44

5. Interactive Tests . . . . . . . . . . . . . . . . . . . . . . 49

5.1 Top Level Interactions $\ldots \ldots \ldots$

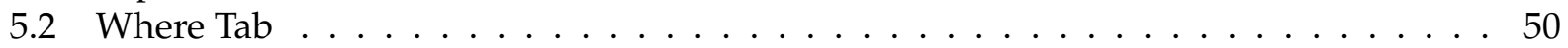

5.3 What Tab . . . . . . . . . . . . . . . . . . . . 51

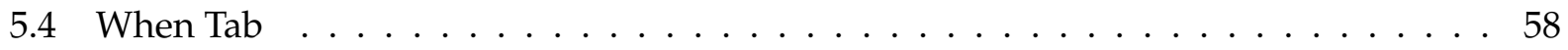

5.5 Notes Tab. . . . . . . . . . . . . . . . . . . . . 62

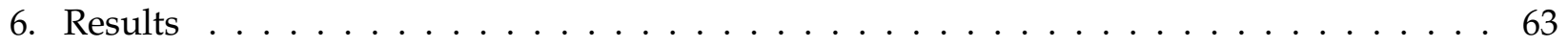

6.1 Automated Test Results . . . . . . . . . . . . . . . . . . . . 63

6.2 Automated Test Coverage . . . . . . . . . . . . . . . . . . . 66

6.3 Interactive Test Results . . . . . . . . . . . . . . . . . . . . . . . . . 69 



\section{LIST OF FIGURES}

Figures $\quad$ Page

$1 \quad$ NFAC Source Terms. . . . . . . . . . . . . . . . . . . 2 



\section{LIST OF TABLES}

\begin{tabular}{|c|c|}
\hline & Page \\
\hline 1 & Multicomponent Material Mode Isotopes \\
\hline 2 & Sub-Release Time Scales . . . . . . . . . . \\
\hline 3 & Code Coverage by Package \\
\hline 4 & $\ldots \ldots \ldots \ldots \ldots$ \\
\hline 5 & Comp.data . \\
\hline 6 & p.data.analyst ..... \\
\hline 7 & $\ldots \ldots \ldots \ldots$ \\
\hline 8 & p.data.times $\ldots \ldots$ \\
\hline 9 & $x_{0}$ \\
\hline 10 & $\mathrm{Coc}$ \\
\hline 11 & Interactions \\
\hline 12 & ( \\
\hline 13 & What Tab \\
\hline 14 & Interactive Test Results: When Tab . . . . . . . . . . . . . . . . . . \\
\hline 15 & Interactive Test Results: Notes $\mathrm{Tab} \ldots \ldots \ldots \ldots$ \\
\hline
\end{tabular}





\section{ACRONYMS}

$\begin{array}{ll}\text { AGR } & \text { Advanced Gas-Cooled Reactor } \\ \text { API } & \text { Application Programming Interface } \\ \text { ATR } & \text { Advanced Thermal Reactor } \\ \text { BWR } & \text { Boiling Water Reactor } \\ \text { DTRA } & \text { Defense Threat Reduction Agency } \\ \text { FBR } & \text { Fast Breeder Reactor } \\ \text { GCHWR } & \text { Gas-Cooled Heavy Water Reactor } \\ \text { GCR } & \text { Gas-Cooled Reactor } \\ \text { GUI } & \text { Graphical User Interface } \\ \text { HPAC } & \text { Hazard Prediction and Assessment Capability } \\ \text { HTGR } & \text { High-Temperature Gas Reactor } \\ \text { HWGCR } & \text { Heavy Water Gas-Cooled Reactor } \\ \text { HWLWR } & \text { Heavy Water Light Water Reactor } \\ \text { LGR } & \text { Light-Water Cooled Graphite-Moderated Reactor } \\ \text { LMFBR } & \text { Liquid-Metal Fast Breeder Reactor } \\ \text { LWR } & \text { Light Water Reactor } \\ \text { MELCOR } & \text { Methods for Estimation of Leakages and Consequences of Releases } \\ \text { MWt } & \text { Megawatt thermal } \\ \text { NFAC } & \text { Nuclear Facility Accident Model } \\ \text { NRC } & \text { Nuclear Regulatory Commission } \\ \text { ORIGEN } & \text { Oak Ridge Isotope Generator } \\ \text { OSGi } & \text { Open Service Gateway Initiative } \\ \text { PHWR } & \text { Pressurized Heavy Water Reactor } \\ \text { PIR } & \text { Percent Inventory Release } \\ \text { RASCAL } & \text { Radiological Assessment Systems for Consequence Analysis } \\ \text { PWR } & \text { Pressurized Water Reactor } \\ \text { RBMK } & \text { (Russian) Reaktor Bolshoy Moschnosti Kanainy } \\ \text { RTH } & \text { Radiological Transport for HPAC } \\ \text { SCIPUFF } & \text { Second-order Closure Integrated Puff Model } \\ \text { SGHWR } & \text { Steam-Generating Heavy Water Reactor } \\ \text { SOARCA } & \text { State-of-the-Art Reactor Consequence Analysis } \\ \text { SPCR } & \text { Software Problem/Change Report } \\ \text { VVER } & \text { (Russian) Water-Water Energy Reactor } \\ & \end{array}$




\section{OVERVIEW}

This document describes the methodology for and results of testing the Nuclear Facility Accident (NFAC) incident source model (ISM) in Version 6.4 of the Hazard Predication and Assessment Capability (HPAC). Tests and results for Version 6.3 have been documented previously. ${ }^{3}$ Individual tests from Version 6.3 that are still relevant are repeated for this report, but the descriptions and source listings of NFAC-specific JUnit test driver components are not repeated and can be found in the prior report.

NFAC's responsibility as an HPAC ISM is as follows. First, it must present an interactive graphical user interface (GUI) by which users can view and edit the definition of an NFAC incident. Second, for each incident defined, NFAC must interact with the Radiological Transport for HPAC (RTH) component to create activity table inputs and associate them with radiological materials to be transported via the Second-order Closure Integrated Puff Model (SCIPUFF). Third, NFAC must create SCIPUFF transport releases and materials and associate them with radiological materials for transport and dispersion. The goal of NFAC unit testing is to verify that these three responsibilities are implemented correctly, and the inputs produced are correct for the source term or model definition as specified.

However, in order for NFAC to function correctly, the other components with which it interacts must process the NFAC-generated inputs in the manner in which NFAC expects. Thus, it is necessary to include NFAC-RTH and NFAC-RTH-SCIPUFF integration tests.

\section{WHAT'S NEW IN 6.4}

Significant changes in NFAC since Version 6.3 are noted below.

Multicomponent material mode. (SPCR 6918) A new material mode has been added to leverage SCIPUFF's multicomponent processing capability. The primary motivation is to approximate the high fidelity of groups mode with orders-of-magnitude improvement in performance. Refer to Section 2.2. Note this new capability coincides with new functionality added to RTH and SCIPUFF for HPAC-6.4.

Improved handling of noble gases in groups material mode. In conjunction with adding multicomponent mode, accounting for noble gases has been corrected and improved when particle groups are used to represent activity in releases.

Improved source term report. The layout of source term reports has been improved to be more consistent with available material and Percent Inventory release modes.

Default spatial domain changed. The default spatial domain for NFAC incidents has been changed to $10 \mathrm{~km}$.

Update to research reactor data. (SPCR 6787) Research reactors have been updated to remove those that were proposed and never built, assign correct names, and add new reactors that have been planned or built. Also, locations have been updated to higher resolution coordinates. 


\section{NFAC DESCRIPTION}

The critical components and capabilities of NFAC Version 6.4 which must be tested are described below.

\subsection{SOURCE MODELS}

As shown in Fig. 1, NFAC contains 22 distinct source term models, all of which are tested.

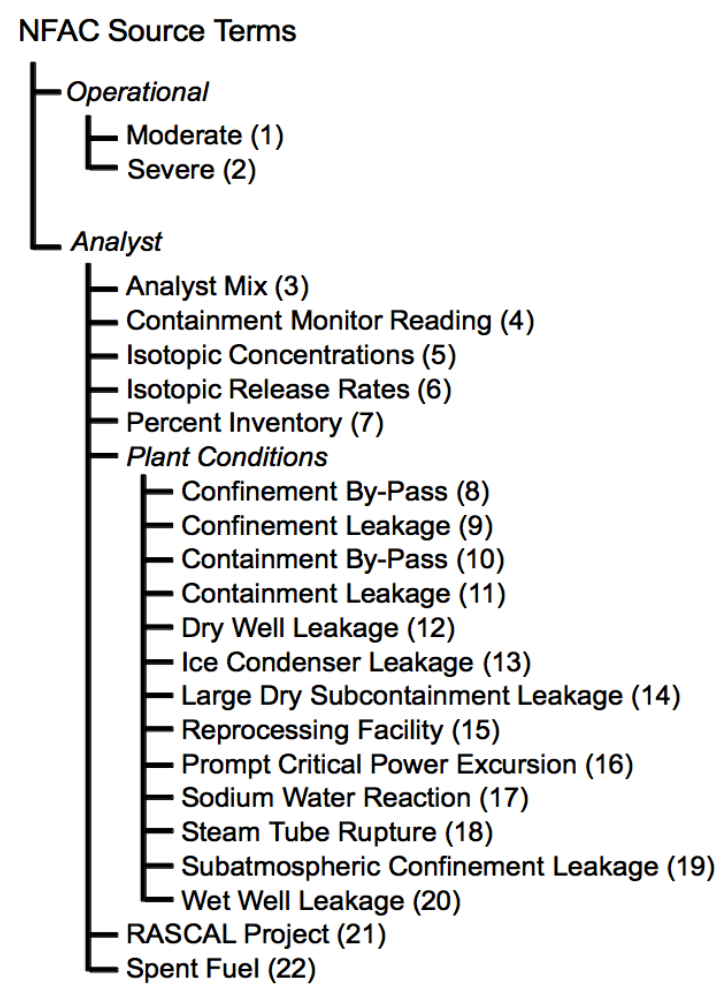

Fig. 1: NFAC Source Terms.

The distinction between operational and analyst models is largely a vestige from HPAC Version 4. The two operational models require minimal user input to define the scenario and are implemented as Percent Inventory source terms. They are based on phased release scenarios defined by the Nuclear Regulatory Commission (NRC).

Percent Inventory is the most widely used and thus the most important source term. Consequently, it is covered more completely in the range of tests. Whereas most models are based on defined accident scenarios, a few are purely for advanced analysis. Each is summarized below.

Moderate. Moderate scale accident defined by the NRC as a series of phased releases specifying percentages of activity released by isotope groups defined by the Methods Estimation of Leakages and Consequences of Releases (MELCOR) code. 


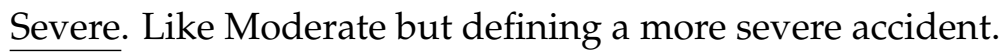

Analyst Mix. Non-scenario mode in which the user specifies the percentage of inventory activity released by MELCOR groups.

Containment Monitor Reading. Scenario defined by readings taken at a containment monitor.

Isotopic Concentrations. Non-scenario mode in which the user specifies what is released as concentrations of specific isotopes.

Isotopic Release Rates. Non-scenario mode in which the user specifies the release as rates for specific isotopes.

Percent Inventory (PIR). Specifies an event as a series of phased releases, which are specifications of percentages by MELCOR group of the inventory released over a specified duration. A shutdown period is represented as an initial duration of no activity released. This is the most used NFAC source term and is used for operational scenarios.

Plant Conditions. A collection of 13 specific accident/event scenarios, the names of which describe the respective scenario. Each requires input of specific parameters to define the event.

RASCAL Project. Imports a project file created with the NRC's Radiological Assessment Systems for Consequence Analysis (RASCAL) application.

Spent Fuel. Specifies a spent fuel pool incident.

NFAC source terms define one or more releases with an activity vector associated with each release. There are variations on how these releases are modeled for transport and dispersion based on nine combinations of three material modes and three PIR release modes, default values for which are simple and none, respectively.

\subsection{MATERIAL MODES}

In terms of radiological materials, NFAC offers three modes for representing the activity associated with each release: simple, groups, multicomponent.

\section{Simple}

The default material mode, simple, offers the fastest calculation but the least fidelity. Each phased release is represented by at most two radiological materials and associated transport releases, one representing depositor isotopes and another representing non-depositors. Noble gases (one of the MELCOR groups) are non-depositors, and other isotopes are depositors. ${ }^{*}$ If the activity vector associated with a phased released contains only depositors, no non-depositor release is created and vice versa.

The gas deposition velocity for the depositor material is $3.0 \mathrm{~mm} / \mathrm{s}$. That is, each depositor isotope is assumed to settle at the same rate.

*The one exception is O-19, a non-depositor that belongs to the chalcogens group. 


\section{Particle Size Groups}

A single deposition velocity for all isotopes has obvious limitations. Thus, NFAC offers a high-fidelity option in which activity is apportioned across as many as 11 particle size groups, each with a specific gas deposition velocity. This groups mode is only available for facilities of the following types:

- Boiling Water Reactor (BWR)

- Light Water Reactor (LWR)

- Pressurized Water Reactor (PWR) (certain variants)

- Water-Water Energy Reactor (VVER)

Apportionment tables are obtained from the State-of-the-Art Reactor Consequence Analysis (SOARCA) studies. BWR tables come from analysis at Peach Bottom, ${ }^{4}$ and tables for the other facility types come from analysis at Surry. ${ }^{5}$ Further, cesium and iodine isotopes are apportioned into cesium iodide (CsI), which is treated as a MELCOR group and for which apportionment ratios are given in the tables. CsI apportionment is also based on SOARCA studies as well as other sources. 6,1

In groups mode, each activity released is represented by as many transport releases and associated transport and radiological materials as there are particle groups with non-zero activity after apportionment, plus a non-depositor release and materials if needed. This geometric growth in the number of materials and releases has an obvious effect on calculation time. Although this mode offers the highest fidelity available in NFAC, it will require a significant increase, sometimes orders of magnitude, in calculation time as compared to simple mode.

\section{Multicomponent}

SCIPUFF has been enhanced for HPAC-6.4 to support definition of multicomponent radiological materials. In conjunction with requisite RTH enhancments, NFAC now offers a third (multicomponent) material mode that leverages this capability. Instead of representing each particle group as a separate material, particle groups are bins associated with "components", which are isotopes. Each transport release associated with an NFAC incident references the same multicomponent radiological material.

NFAC passes a single activity vector to RTH for the latter to use in calculating dose factor tables to write to the radfile that is read by SCIPUFF. In this new scheme, a basis value of $1.0 \mathrm{Ci}$ is set as the activity for each isotope in the vector. In effect, the radfile dose factor tables are basis factors. Also written to the radfile are the particle group sizes and deposition velocities as well as names for each isotope representing a "component". The association of the actual activity for each isotope in each particle group or bin is now in the transport release. That is, each transport release created by NFAC for an incident references the same radiological material but includes "mass" factors to apply for each isotope and group/bin. This representation allows SCIPUFF to calculate the transport and deposition and account for radiological dose with much less computational overhead. 
Table 1: Multicomponent Material Mode Isotopes

\begin{tabular}{llllll}
\hline \hline MELCOR Group & Isotopes & & & \\
\hline Noble Gases & Kr-85 & Xe-133 & Xe-135 & & \\
Alkali Metals & Cs-134 & Cs-137 & & & \\
Alkaline Earths & Ba-140 & Sr-89 & Sr-90 & & \\
Halogens & $\mathrm{I}-131$ & & & & \\
Chalcogens & Te-129 & & & & \\
Platinoids & Ru-103 & Ru-106 & & & \\
Early Transition Elements & Mo-99 & & & & \\
Tetravalents & Ce-141 & Ce-144 & Pu-239 & Zr-95 & \\
Trivalents & La-140 & Nb-95 & Nd-147 & Pr-143 & Y-91 \\
\hline
\end{tabular}

However, there is a limitation on the number of "components" that SCIPUFF can process efficiently. Consequently, NFAC limits the activity vector to a list of 22 isotopes that represent the lion's share of activity and effects. They are listed in Table 1.

It is important to note that NFAC's multicomponent material mode is a close approximation to groups mode that requires significantly less calculation time. However, activity is not preserved in in multicomponent mode.

\subsection{PIR RELEASE MODES}

As described above, PIR scenarios are specified as a series of phased releases with an associated duration. The activity vectors (lists of isotopes and associated activity) associated with each phased release are determined by decaying the inventory to the start of the release. For a long duration phase (e.g., $48 \mathrm{~h}$ ), there are many daughter products which will never be accounted for due to their appearance and decay during the period. Even shorter duration phases early in the series can lose significant effects due to lost daughter products. NFAC offers three release modes, two of which offer an accounting for daughter products in these situations. In order of increasing calculation time and fidelity, the modes are: none, average, and explicit.

For average and explicit modes, each phased is divided into sub-releases as shown in Table 2.

Table 2: Sub-Release Time Scales

\begin{tabular}{rrrr}
\hline \hline Time in Phase & Sub-Phase Duration & Sub-Release Duration & No. Releases \\
\hline 0.0 & 3.0 & 0.5 & $+6=6$ \\
3.0 & 3.0 & 1.0 & $+3=9$ \\
6.0 & 9.0 & 3.0 & $+3=12$ \\
15.0 & 36.0 & 12.0 & $+3=15$ \\
51.0 & - & 24.0 & \\
\hline
\end{tabular}

(Times are in hours)

For the first $3.0 \mathrm{~h}$ of a phased release, a subrelease is created every $0.5 \mathrm{~h}$, resulting in a maximum six releases. For the next $3.0 \mathrm{~h}$, a subrelease is created every $1.0 \mathrm{~h}$, for an additional three releases. Thus, a phased release of $6.0 \mathrm{~h}$ will have nine subreleases, six with a duration of $0.5 \mathrm{~h}$, and three with a duration of $1.0 \mathrm{~h}$. 
Over the next $9.0 \mathrm{~h}$, a subrelease is created every $3.0 \mathrm{~h}$ (an additional three), and over the next $36.0 \mathrm{~h}$, a subrelease is created every $12.0 \mathrm{~h}$ (another three). After $51.0 \mathrm{~h}$, a subrelease is created for every $24.0 \mathrm{~h}$. For example, a phase with duration $72.0 \mathrm{~h}$ will have 15 releases representing the first $51.0 \mathrm{~h}$ and an additional release of $21.0 \mathrm{~h}$. A phase of $12.0 \mathrm{~h}$ duration will have 11 releases, nine for the first $6.0 \mathrm{~h}$ and additional two for the next $6.0 \mathrm{~h}$.

None. This is the default. No subreleases are created, and thus there is no accounting for lost daughter products.

Explicit. A transport release and associated transport and radiological materials are created for each subrelease. Although this mode offers the highest fidelity, it can greatly increase the SCIPUFF calculation time.

Average. This is a reasonable compromise that avoids long calculation times for SCIPUFF. Activities in subreleases are averaged with subrelease durations as weights. The resulting activity vector is applied to the original release.

\section{TEST APPROACH}

Although NFAC unit tests comprise most of the tests conducted, testing includes NFAC-RTH and NFAC-RTH-SCIPUFF integration tests. The integration tests are are necessary to verify NFAC is functioning as designed.

Testing is divided into two paths: automated tests and interactive tests. Nine automated JUnit4 test suites containing 150 individual tests have been built and are described in detail below. Each individual test includes one or more assertions challenging specific results or consequences designed to exercise a key functional capability. The granularity of the capability being tested varies by test from an individual component method to an aggregate or composite test involving multiple components and methods. Success or failure of automated tests is an output of the test framework. They are run as unattended batch processes.

The GUI is not conducive to automated tests and thus must be tested interactively. All capabilities in the GUI are tested explicitly as described below.

\subsection{NFAC UNIT TESTS}

Some automated NFAC unit tests rely on aggregate information written by NFAC components to the log file. The log output contains all the data regarding activity table inputs fed to RTH and releases created for SCIPUFF and thus can be compared with baseline logs for equivalence.

\subsection{NFAC-RTH INTEGRATION TESTS}

For NFAC-RTH integration, two kinds of test artifacts are used in comparisons:

- Source term report HTML files

- Radfiles 
Report files are created from information retrieved from RTH by NFAC. That is, NFAC asks RTH to return information previously passed to RTH by NFAC. Radfiles are generated by RTH as requested by SCIPUFF when a calculation is requested.

\subsection{NFAC-RTH-SCIPUFF INTEGRATION TESTS}

Some tests use artifacts that are created by SCIPUFF after transport and dispersion calculation. These are outputs based on plots requested via an HPAC OutputManager instance.

\section{AUTOMATED TESTS}

\subsection{EXTERNALFILEFINDERTEST}

This suite is composed of four individual tests to verify correct function of NFAC's PropertyFinder class used to identify and locate ExternalFile instances to be (re)stored for a project. PropertyFinder instances are used by Nfaclmpl instances in exportFiles() and importFiles() methods now required for correct HPAC project export and import operations, respectively. Tests in alphabetical order are summarized below.

NFAC components tested:

mil.dtra.hpac.models.nfac.CAcomp.data.FacilityDef

mil.dtra.hpac.models.nfac.CAcomp.data.Nfaclncident

mil.dtra.hpac.models.nfac.CAcomp.data.PropertyFinder

mil.dtra.hpac.models.nfac.CAcomp.impl.Nfaclmpl

\section{Test: testAllIncidents}

Loads a project containing multiple Nfaclmpl instances, assigns a custom inventory with an ExternalFile reference to each instance, and invokes the finder() method of a PropertyFinder instance to locate the ExternalFiles. Verifies all ExternalFiles are found.

\section{Test: testFileReleases}

Loads a project containing FileRelease instances and ensures all associated ExternalFiles are found.

\section{Test: testNfacImpl}

Tests correct behavior of the Nfaclmpl.exportFiles() method.

\section{Test: testProject}

Loads a project with an NFAC incident with a custom inventory and verifies that the findExternalFiles() method of PropertyFinder behaves correctly. 


\subsection{MODELDEFSTEST}

This suite contains 35 individual tests designed to verify proper function of each of the NFAC source models. For each test there is a corresponding HPAC 6 project (.hpac6) file or a project export (.zip) file. For most tests a source term report $(. h t m l)$ is requested and compared to the baseline fixture, and when appropriate a $\log$ file $(. \log )$ is an additional fixture.

A ProjectManager instance is used to open the project file. The Nfaclmp instance(s) in the project are retrieved, and the getSourceTermReport() method is called to generated the HTML report. The test and baseline reports are compared for equivalence, allowing for different seed values used in material names. For those tests including a log file fixture, the fixture and $\log$ file resulting from the run are read into NfacLog objects and compared with the NfacLog.equals() method. Exact matches determine a successful test. Any difference in report content or NfacLog objects represents a test failure. Tests in alphabetical order are summarized below.

NFAC components tested:

mil.dtra.hpac.models.nfac.CAcomp.data.ActivityHash

mil.dtra.hpac.models.nfac.CAcomp.data.AmbClient

mil.dtra.hpac.models.nfac.CAcomp.data.Element

mil.dtra.hpac.models.nfac.CAcomp.data. Facility

mil.dtra.hpac.models.nfac.CAcomp.data.FacilityDB

mil.dtra.hpac.models.nfac.CAcomp.data.FacilityDBMgr

mil.dtra.hpac.models.nfac.CAcomp.data.FacilityDef

mil.dtra.hpac.models.nfac.CAcomp.data.MaterialMode

mil.dtra.hpac.models.nfac.CAcomp.data.ModelDefUtils

mil.dtra.hpac.models.nfac.CAcomp.data.ModelTimes

mil.dtra.hpac.models.nfac.CAcomp.data.ModerateModel

mil.dtra.hpac.models.nfac.CAcomp.data.Nfaclncident

mil.dtra.hpac.models.nfac.CAcomp.data.NfaclncidentMgr

mil.dtra.hpac.models.nfac.CAcomp.data.NfacRelease

mil.dtra.hpac.models.nfac.CAcomp.data.Options

mil.dtra.hpac.models.nfac.CAcomp.data.ParticleGroups

mil.dtra.hpac.models.nfac.CAcomp.data.ParticleGroupsMgr

mil.dtra.hpac.models.nfac.CAcomp.data.Reprolnventory

mil.dtra.hpac.models.nfac.CAcomp.data.SevereModel

mil.dtra.hpac.models.nfac.CAcomp.data.analyst.AnalystMix

mil.dtra.hpac.models.nfac.CAcomp.data.analyst.AnalystModelUtils

mil.dtra.hpac.models.nfac.CAcomp.data.analyst.ConcentrationUnits

mil.dtra.hpac.models.nfac.CAcomp.data.analyst.ContainmentMonitorReading

mil.dtra.hpac.models.nfac.CAcomp.data.analyst.IsotopeReleases

mil.dtra.hpac.models.nfac.CAcomp.data.analyst.IsotopeValue

mil.dtra.hpac.models.nfac.CAcomp.data.analyst.IsotopicConcentrations

mil.dtra.hpac.models.nfac.CAcomp.data.analyst.IsotopicReleaseRates

mil.dtra.hpac.models.nfac.CAcomp.data.analyst.PIRSubReleaseMode

mil.dtra.hpac.models.nfac.CAcomp.data.analyst.PercentInventory

mil.dtra.hpac.models.nfac.CAcomp.data.analyst.PercentInventoryConstants

mil.dtra.hpac.models.nfac.CAcomp.data.analyst.PercentInventoryRelease 
mil.dtra.hpac.models.nfac.CAcomp.data.analyst.PercentInventoryReleaseTree

mil.dtra.hpac.models.nfac.CAcomp.data.analyst.RascalFileReader

mil.dtra.hpac.models.nfac.CAcomp.data.analyst.RascalProject

mil.dtra.hpac.models.nfac.CAcomp.data.analyst.RascalXmIReader

mil.dtra.hpac.models.nfac.CAcomp.data.analyst.ReleaseRate

mil.dtra.hpac.models.nfac.CAcomp.data.analyst.ReleaseRateUnits

mil.dtra.hpac.models.nfac.CAcomp.data.analyst.SpentFuel

mil.dtra.hpac.models.nfac.CAcomp.data.analyst.plantcond.PCConfinementByPass

mil.dtra.hpac.models.nfac.CAcomp.data.analyst.plantcond.PCConfinementLeakage

mil.dtra.hpac.models.nfac.CAcomp.data.analyst.plantcond.PCContainmentByPass

mil.dtra.hpac.models.nfac.CAcomp.data.analyst.plantcond.PCContainmentLeakage

mil.dtra.hpac.models.nfac.CAcomp.data.analyst.plantcond.PCDryWellLeakage

mil.dtra.hpac.models.nfac.CAcomp.data.analyst.plantcond.PClceCondenserContainment

...models.nfac.CAcomp.data.analyst.plantcond.PCLargeDrySubContainmentLeakage

...models.nfac.CAcomp.data.analyst.plantcond.PCPromptCriticalPowerExcursion

mil.dtra.hpac.models.nfac.CAcomp.data.analyst.plantcond.PCReproFacility

mil.dtra.hpac.models.nfac.CAcomp.data.analyst.plantcond.PCWaterReaction

mil.dtra.hpac.models.nfac.CAcomp.data.analyst.plantcond.PCSteamTubeRupture

...models.nfac.CAcomp.data.analyst.plantcond.PCSubatmosphericConfinementLeakage

mil.dtra.hpac.models.nfac.CAcomp.data.analyst.plantcond.PCWetWellLeakaage

mil.dtra.hpac.models.nfac.CAcomp.impl.MelcorFraction

mil.dtra.hpac.models.nfac.CAcomp.impl.MelcorFractions

mil.dtra.hpac.models.nfac.CAcomp.impl.MelcorReleaseFractionsFile

mil.dtra.hpac.models.nfac.CAcomp.impl.Nfaclmpl

mil.dtra.hpac.models.nfac.CAcomp.impl.PercentInventoryFileMgr

mil.dtra.hpac.models.nfac.CAcomp.impl.RepoData

mil.dtra.hpac.models.nfac.CAcomp.impl.ReproMod

mil.dtra.hpac.models.nfac.CAcomp.impl.StcalcClient

Test: test10682GroupsExplicit

Tests the new capabilities added for SPCR 10682. Refer to Section 2.2 for a description of the material modes. This test ensures the activity apportionment across particle groups is correct and the corresponding materials are created with the groups material mode and the explicit PIR release mode. 


\begin{tabular}{|c|c|c|c|}
\hline & ility: & & Peach Bottom-2 (BWR Mk-1) \\
\hline & erating $\mathrm{Pc}$ & ver: & $3414.0 \mathrm{MWt}$ \\
\hline & terial Moc & & Groups \\
\hline & Release & Mode: & Explicit \\
\hline & arce Term & & Percent Inventory \\
\hline & utdown D & ration: & 0 \\
\hline & $\begin{array}{l}\text { ease Dur } \\
\text { eases: }\end{array}$ & tion: & $7.0 \mathrm{~h}$ \\
\hline \# & Duration & Perce & htages by Group \\
\hline 0 & $0.5 \mathrm{~h}$ & Noble & Gas $=0.01$, AlkaMetal $=0.6$, Chalcogen $=0.35$ \\
\hline 1 & $2.0 \mathrm{~h}$ & Noble & Gas $=0.09$, AlkaMetal=1.7, AlkaEarth=0.03, Chalcogen $=1.45$ \\
\hline 2 & $4.5 \mathrm{~h}$ & Nobel & Gas $=0.8$, AlkaMetal $=0.9$, Chalcogen $=0.45$ \\
\hline
\end{tabular}

Test: test10682GroupsNone

Tests proper activity apportionment and material creation for the groups material mode and none PIR release mode.

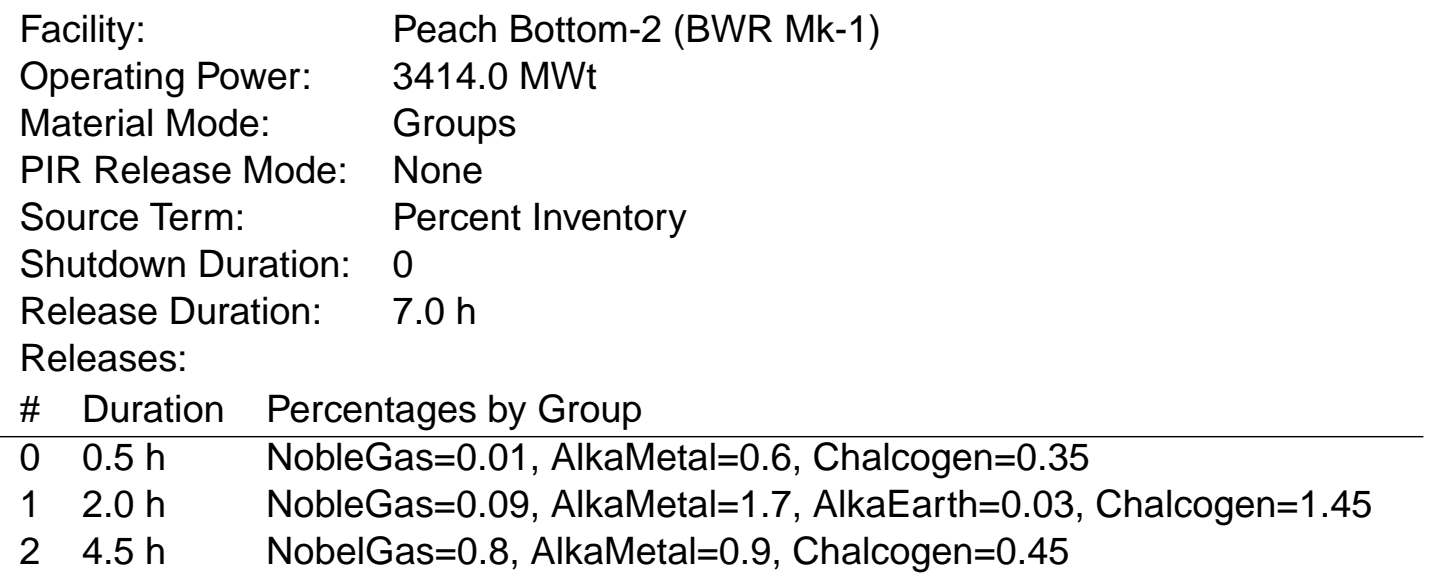

Test: test10682PwrGroupsNone

Tests proper activity apportionment and material creation for the groups material mode and none PIR release mode. 


\begin{tabular}{|c|c|c|c|}
\hline \multicolumn{3}{|c|}{ Facility: } & Arkansas One-1 (PWR) \\
\hline \multirow{2}{*}{\multicolumn{3}{|c|}{$\begin{array}{l}\text { Operating Power: } \\
\text { Material Mode: }\end{array}$}} & $2550.0 \mathrm{MWt}$ \\
\hline & & & Groups \\
\hline \multicolumn{3}{|c|}{ PIR Release Mode: } & None \\
\hline \multicolumn{3}{|c|}{ Source Term: } & Percent Inventory \\
\hline \multirow{2}{*}{\multicolumn{3}{|c|}{$\begin{array}{l}\text { Shutdown Duration: } \\
\text { Release Duration: }\end{array}$}} & $1.0 \mathrm{~h}$ \\
\hline & \multicolumn{3}{|c|}{ Releases: } \\
\hline \# & Duration & \multicolumn{2}{|c|}{ Percentages by Group } \\
\hline 0 & $1.0 \mathrm{~h}$ & \multicolumn{2}{|r|}{0} \\
\hline 1 & $5.0 \mathrm{~h}$ & \multicolumn{2}{|r|}{$\begin{array}{l}\text { NobleGas }=3.0, \text { AlkaMetal }=0.003, \text { Halogens }=0.003 \text {, Chalcogen }=0.03 \text {, } \\
\text { Platinoid }=1.68 \mathrm{e}-6 \text {, Tetravalent }=4.0 \mathrm{e}-7 \text {, Trivalent }=6.0 \mathrm{e}-7\end{array}$} \\
\hline 2 & $2.0 \mathrm{~h}$ & \multicolumn{2}{|r|}{$\begin{array}{l}\text { NobleGas }=17, \text { AlkaMetal=0.07, AlkaEarth }=8.95 e-5, \text { Halogens }=0.089, \\
\text { Chalcogen=0.64,Platinoid=6.2e-6, Tetravalent }=7.0 \mathrm{e}-7, \text { Trivalent }=1.6 \mathrm{e}-6\end{array}$} \\
\hline
\end{tabular}

\section{Test: test10682RascalGroupsNone}

Tests proper activity apportionment and material creation for a RASCAL source term and the groups material mode.

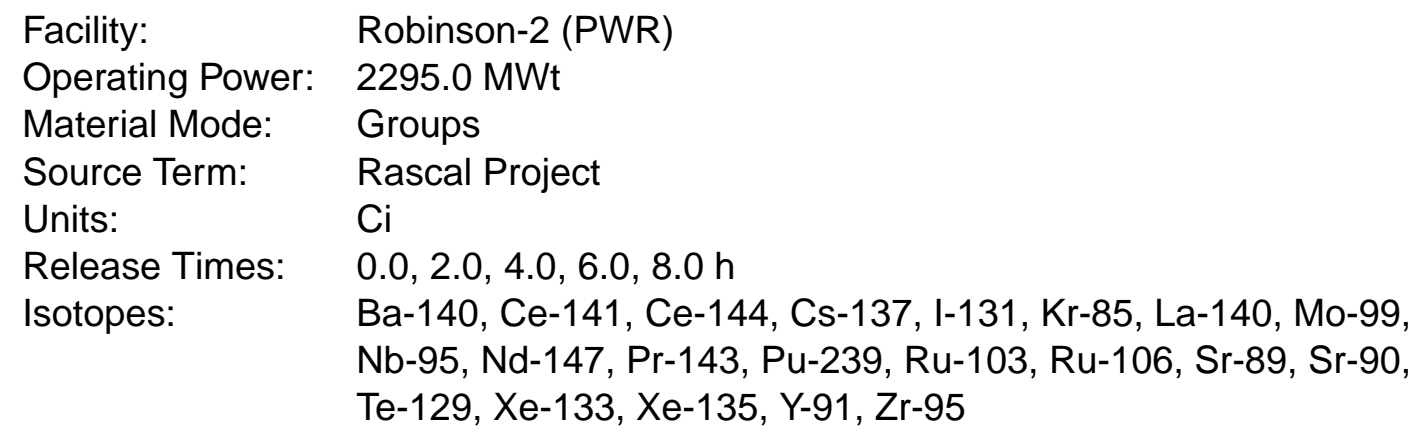

\section{Test: test10682RascalSimpleNone}

Tests proper activity apportionment and material creation for a RASCAL source term and the groups material mode.

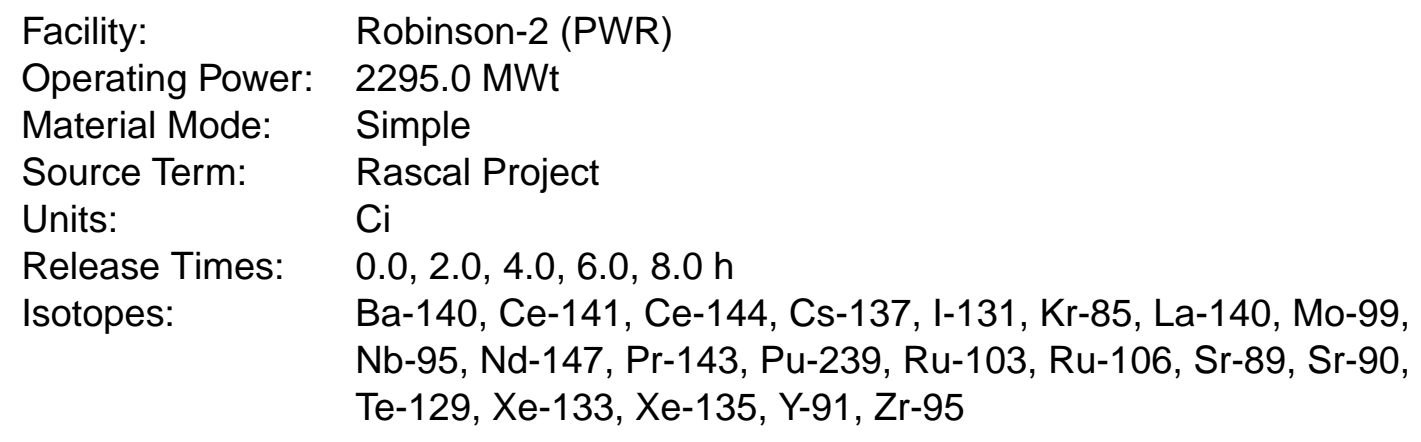




\section{Test: test10682SimpleExplicit}

Tests that activity apportionment and per-particle-size material creation do not occur for a scenario using the explicit PIR release mode.

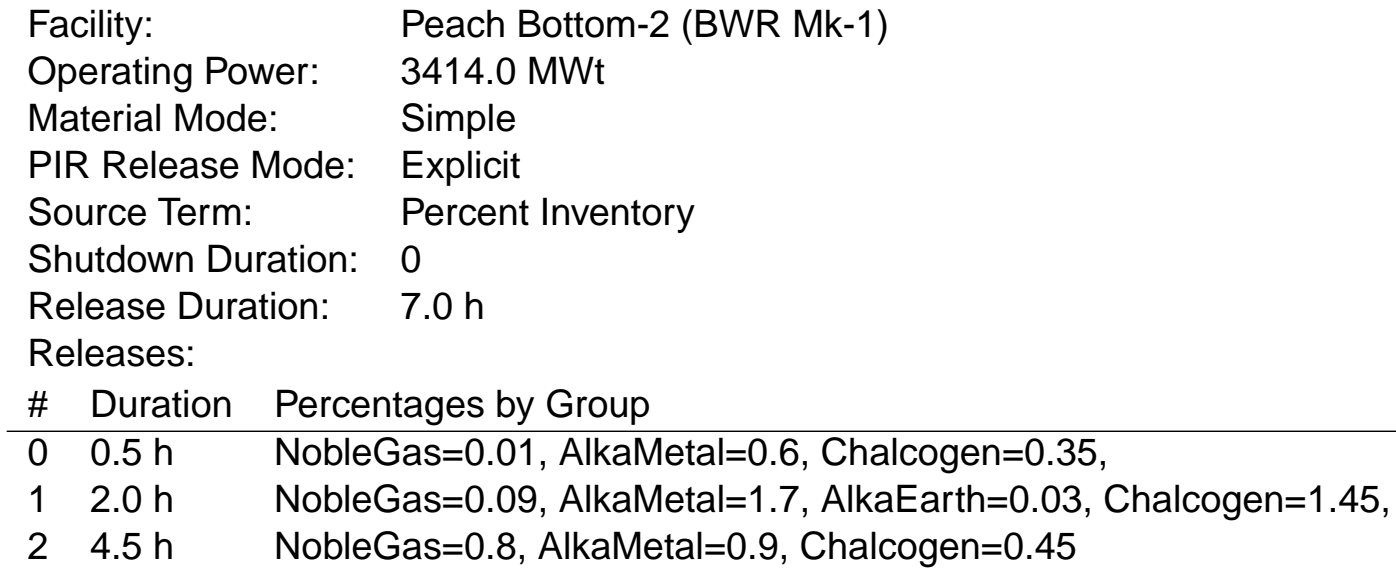

Test: test10682SimpleNone

Tests that activity apportionment and per-particle-size material creation do not occur for a scenario using the none PIR release mode.

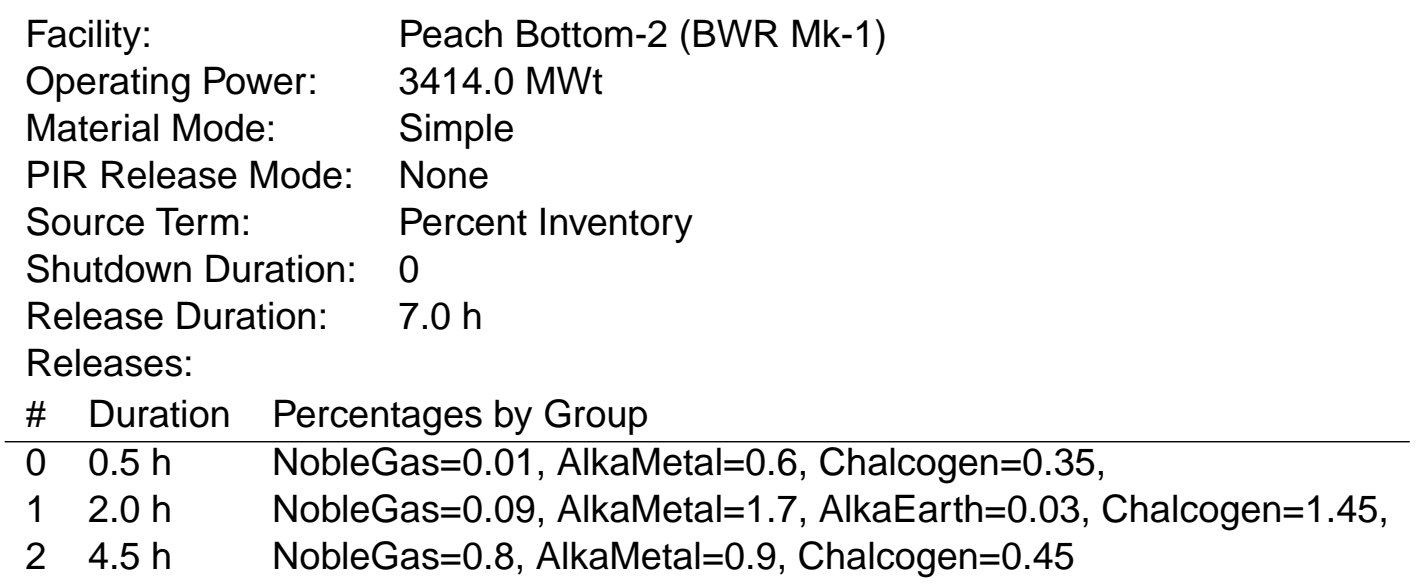

\section{Test: test10682UnsupportedGroupsNone}

Tests that activity apportionment and per-particle-size material creation do not occur even though requested via the groups material mode for an unsupported facility type and the none PIR release mode. 


\begin{tabular}{|c|c|c|c|}
\hline \multicolumn{3}{|c|}{ Facility: } & Hartlepool-1 (AGR) \\
\hline \multicolumn{3}{|c|}{ Operating Power: } & $1785.0 \mathrm{MWt}$ \\
\hline \multirow{2}{*}{\multicolumn{3}{|c|}{$\begin{array}{l}\text { Material Mode: } \\
\text { PIR Release Mode: }\end{array}$}} & disabled \\
\hline & Release & Mode: & None \\
\hline \multicolumn{3}{|c|}{ Source Term: } & Percent Inventory \\
\hline \multicolumn{3}{|c|}{ Shutdown Duration: } & $1.0 \mathrm{~h}$ \\
\hline \multirow{2}{*}{\multicolumn{4}{|c|}{ Releases: }} \\
\hline & & & \\
\hline & Duration & \multicolumn{2}{|c|}{ Percentages by Group } \\
\hline & $1.0 \mathrm{~h}$ & \\
\hline & $2.0 \mathrm{~h}$ & \multicolumn{2}{|r|}{$\begin{array}{l}\text { NobleGas }=3.0 \text {, AlkaMetal }=0.003 \text {, Halogens }=0.003 \text {, Chalcogen }=0.03 \text {, } \\
\text { Platinoid }=1.68 \mathrm{e}-6 \text {, Tetravalent }=4.0 \mathrm{e}-7 \text {, Trivalent }=6.0 \mathrm{e}-7\end{array}$} \\
\hline & $5.0 \mathrm{~h}$ & \multicolumn{2}{|r|}{$\begin{array}{l}\text { NobleGas }=17 \text {, AlkaMetal }=0.07 \text {, AlkaEarth }=8.95 \mathrm{e}-5 \text {, Chalcogen }=0.64 \text {, } \\
\text { Platinoid }=6.2 \mathrm{e}-6 \text {, Tetravalent }=7.0 \mathrm{e}-7 \text {, Trivalent }=1.6 \mathrm{e}-6\end{array}$} \\
\hline
\end{tabular}

Test: test10682VverGroupsNone

Tests proper activity apportionment and material creation occurs for a VVER facility based on the model for a PWR using the groups material mode and the none PIR release mode.

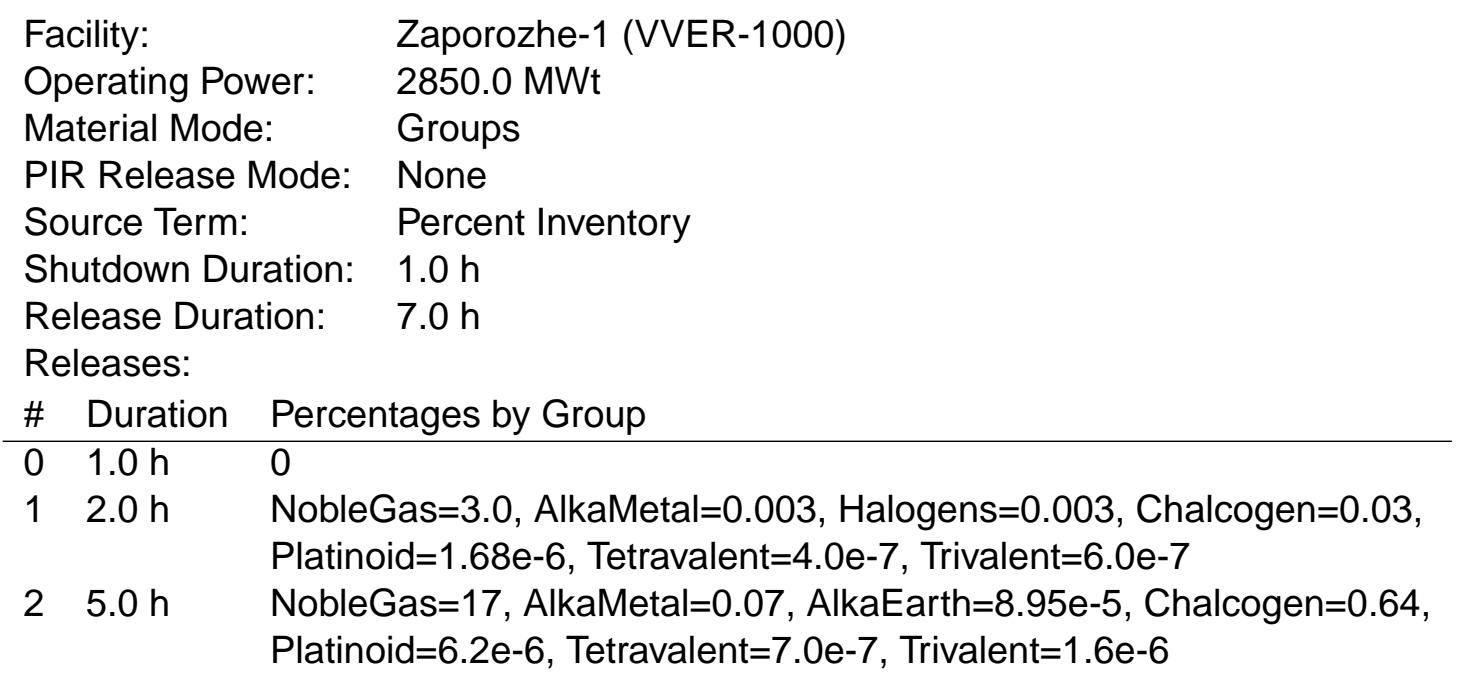

\section{Test: test11653}

Explicitly tests the fix for SPCR 11653 to ensure a custom inventory is accounted for when determining the available plant conditions source terms. 


$\begin{array}{ll}\text { Facility: } & \text { Arkansas One-1 (PWR) } \\ \text { Operating Power: } & \text { 2550.0 MWt } \\ \text { Custom Inventory: } & \text { Created from kewaunee.f71 ORIGEN file } \\ \text { Material Mode: } & \text { Simple } \\ \text { Source Term: } & \text { Mix Specified by Analyst } \\ \text { Gross Release Rate: } & 1.0 \mathrm{Ci} / \mathrm{s} \\ \text { Release Percentages: } & \\ \text { Nobel Gases: } \quad 98.0 \% & \\ \text { Halogens: } \quad 2.0 \% & \end{array}$

\section{Test: testAnalystMix}

Tests an AnalystMix model instance with the following properties:

$\begin{array}{llll}\text { Facility: } & \text { Brunswick-1 (BWR Mk-1) } & \\ \text { Operating Power: } & 2949.0 \mathrm{MWt} & \\ \text { Material Mode: } & \text { Simple } & \text { Nobel Gases: } & 98.0 \% \\ \text { Source Term: } & \text { Mix Specified by Analyst } & \text { Halogens: } & 2.0 \% \\ \text { Gross Release Rate: } & 100.0 \mathrm{Ci} / \mathrm{s} & & \\ \text { Release Percentages: } & & \end{array}$

Test: testContainmentMonitorReading

Tests a ContainmentMonitorReading model instance with the following properties:

$\begin{array}{ll}\text { Facility: } & \text { Brunswick-1 (BWR Mk-1) } \\ \text { Operating Power: } & 2949.0 \mathrm{MWt} \\ \text { Material Mode: } & \text { Simple } \\ \text { Source Term: } & \text { Containment Monitor Reading } \\ \text { Leak Rate: } & 10 \% / \mathrm{h} \\ \text { Monitor Location: } & \text { Wet Well } \\ \text { Monitor Reading: } & 25.0 \mathrm{R} / \mathrm{h} \\ \text { Release Path: } & \text { Unfiltered } \\ \text { Sprays: } & \text { Off }\end{array}$

Test: testIsotopicConcentrations

Tests a IsotopicConcentrations model instance with the following properties:

Facility: $\quad$ Brunswick-1 (BWR Mk-1)

$\begin{array}{llll}\text { Operating Power: } & 2949.0 \mathrm{MWt} & \text { Cs-130: } & 130.0 \\ \text { Material Mode: } & \text { Simple } & \text { Cs-131: } & 131.0 \\ \text { Source Term: } & \text { Isotopic Concentrations } & \text { I-130: } & 130.0 \\ \text { Concentration Units: } & \mathrm{kCi} / \mathrm{cc} & \mathrm{I}-132: & 132.0 \\ \text { Release Rate: } & 20.0 \mathrm{cc} / \mathrm{s} & \mathrm{I}-133: & 133.0 \\ \text { Isotope Values: } & & & \end{array}$




\section{Test: testIsotopicReleaseRates}

Tests a IsotopicReleaseRates model instance with the following properties:

$\begin{array}{llll} & & \text { Cs-130: } & 130.0 \\ \text { Facility: } & \text { Brunswick-1 (BWR Mk-1) } & \text { Cs-131: } & 131.0 \\ \text { Operating Power: } & 2949.0 \mathrm{MWt} & \text { Cs-132: } & 132.0 \\ \text { Material Mode: } & \text { Simple } & \text { Cs-134: } & 134.0 \\ \text { Source Term: } & \text { Isotopic Release Rates } & \text { I-130: } & 130.0 \\ \text { Release Units: } & \mathrm{kCi} / \mathrm{s} & \mathrm{I}-131: & 131.0 \\ \text { Isotope Values: } & & \mathrm{l}-132: & 132.0 \\ & & \mathrm{l}-133: & 133.0\end{array}$

Test: testModerate

Tests an operational ModerateModel instance with properties:

Facility: $\quad$ Brunswick-1 (BWR Mk-1)

Operating Power: $2949.0 \mathrm{MWt}$

Test: testMultiFive

Tests a project with five NFAC incidents.

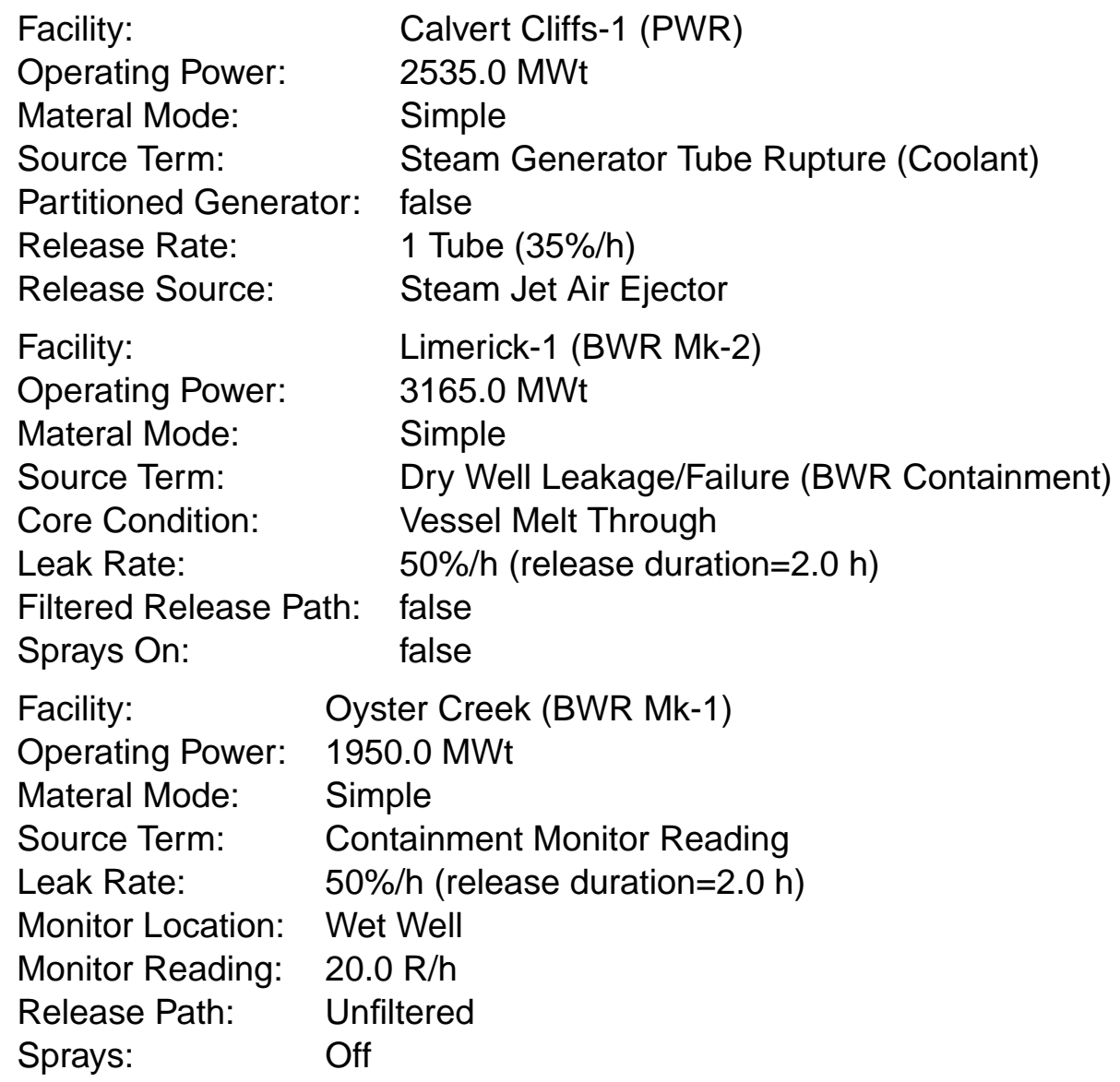




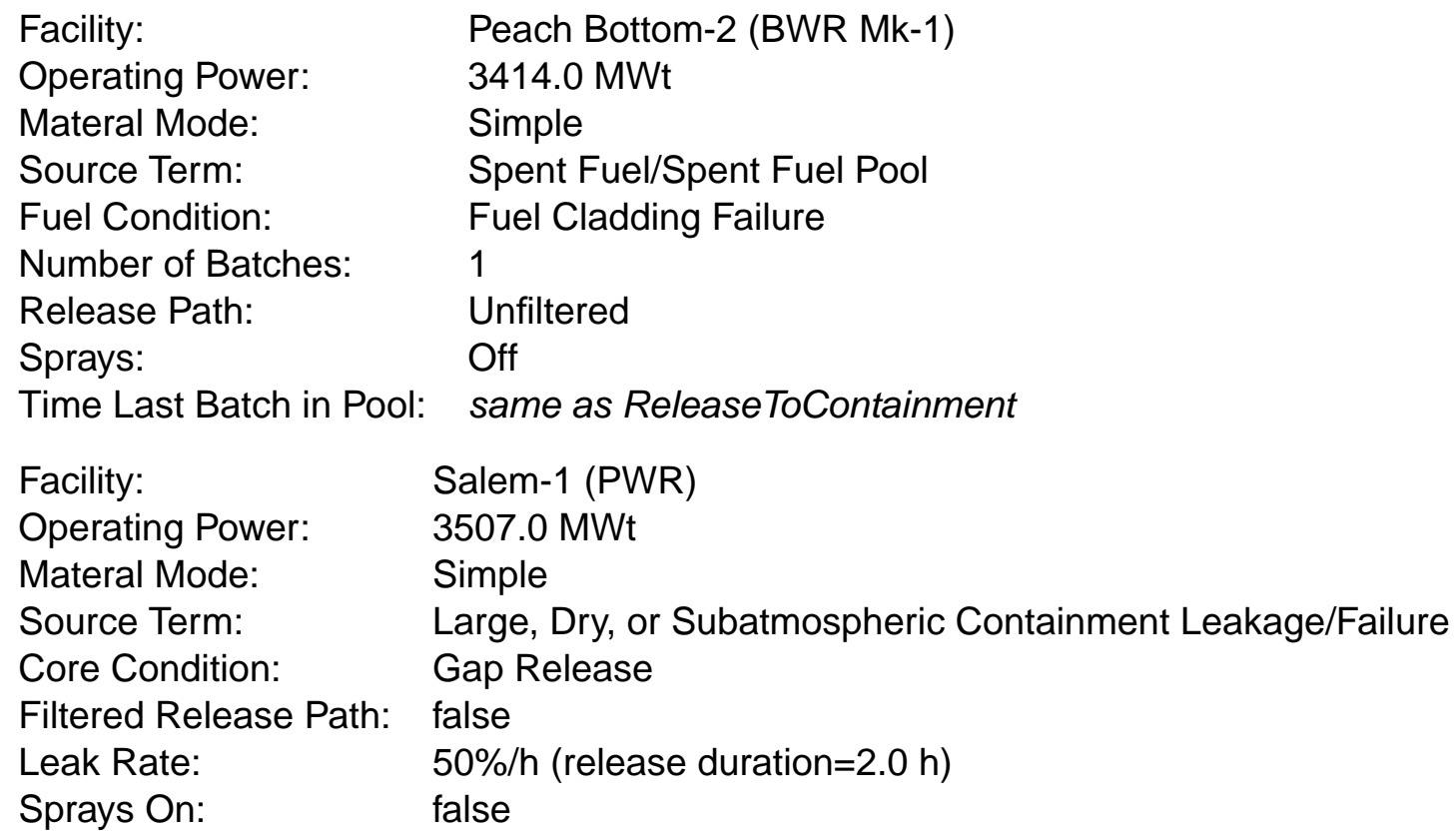

\section{Test: testPCConfinementByPass}

Tests a PCConfinementByPass plant conditions model instance with properties:

$\begin{array}{ll}\text { Facility: } & \text { Hartlepool-1 (AGR) } \\ \text { Operating Power: } & 1785.0 \mathrm{MWt} \\ \text { Material Mode: } & \text { disabled } \\ \text { Source Term: } & \text { Plant Conditions: Bypass of Confinement } \\ \text { Core Condition: } & \text { Gap Release } \\ \text { Leak Rate: } & 25 \% / \mathrm{h} \text { (release duration=4.0 h) }\end{array}$

Test: testPCConfinementLeakage

Tests a PCConfinementLeakage plant conditions model instance with properties:

$\begin{array}{ll}\text { Facility: } & \text { Novovoronezh-3 (VVER-400/230) } \\ \text { Operating Power: } & 1155.0 \mathrm{MWt} \\ \text { Material Mode: } & \text { Simple } \\ \text { Source Term: } & \text { Plant Conditions: Confinement Leakage/Failure } \\ \text { Core Condition: } & \text { Gap Release } \\ \text { Leak Rate: } & 50 \% / \mathrm{h} \text { (release duration=2.0 h) }\end{array}$

Test: testPCContainmentByPass

Tests a PCContainmentByPass plant conditions model instance with properties: 
Facility:

Hamaoka-3 (BWR Mk-1)

Operating Power:

3168.0 MWt

Material Mode:

Simple

Source Term:

Core Condition:

Plant Conditions: Bypass of Containment

Leak Rate:

Gap Release

$100 \% / \mathrm{h}$ (release duration $=1.0 \mathrm{~h}$ )

Filtered Release Path: false

\section{Test: testPCContainmentLeakage}

Tests a PCContainmentLeakage plant conditions model instance with properties:

Facility:

Operating Power:

Material Mode:

Source Term:

Core Condition:

Leak Rate:

Filtered Release Path:
Beloyarski-3 (BN-600) (LMFBR)

1680.0 MWt

disabled

Plant Conditions: Containment Leakage/Failure

Gap Release

$50 \% / \mathrm{h}$ (release duration $=2.0 \mathrm{~h}$ )

false

Test: testPCDryWellLeakage

Tests a PCDryWellLeakage plant conditions model instance with properties:

$\begin{array}{ll}\text { Facility: } & \text { Hamaoka-3 (BWR Mk-1) } \\ \text { Operating Power: } & 3168.0 \mathrm{MWt} \\ \text { Material Mode: } & \text { Simple } \\ \text { Source Term: } & \text { Plant Conditions: Dry Well Leakage/Failure (BWR Containment) } \\ \text { Core Condition: } & \text { Gap Release } \\ \text { Filtered Release Path: } & \text { false } \\ \text { Leak Rate: } & 100 \% / \mathrm{h} \text { (release duration }=1.0 \mathrm{~h}) \\ \text { Sprays On: } & \text { false }\end{array}$

Test: testPCIceCondenserContainmentCoreDamage

Tests a PClceCondenserContainment plant conditions model instance with properties:

$\begin{array}{ll}\text { Facility: } & \text { Watts Bar-1 (PWR) } \\ \text { Operating Power: } & 3465.0 \mathrm{MWt} \\ \text { Material Mode: } & \text { Simple } \\ \text { Source Term: } & \text { Plant Conditions: Ice Condenser Containment Leakage/Failure } \\ \text { Core Condition: } & \text { In-Vessel Severe Core Damage } \\ \text { Fans On: } & \text { true } \\ \text { Filtered Release Path: } & \text { true } \\ \text { lce Bed Exhausted: } & \text { true } \\ \text { Leak Rate: } & 50 \% / \mathrm{h} \text { (release duration=2.0 h) } \\ \text { Sprays On: } & \text { true }\end{array}$




\section{Test: testPCIceCondenserContainmentGapRelease}

Tests a PClceCondenserContainment plant conditions model instance with properties:

$\begin{array}{ll}\text { Facility: } & \text { Watts Bar-1 (PWR) } \\ \text { Operating Power: } & 3465.0 \mathrm{MWt} \\ \text { Material Mode: } & \text { Simple } \\ \text { Source Term: } & \text { Plant Conditions: Ice Condenser Containment Leakage/Failure } \\ \text { Core Condition: } & \text { Gap Release } \\ \text { Fans On: } & \text { false } \\ \text { Filtered Release Path: } & \text { false } \\ \text { lce Bed Exhausted: } & \text { false } \\ \text { Leak Rate: } & 50 \% / h \text { (release duration=2.0 h) } \\ \text { Sprays On: } & \text { false }\end{array}$

Test: testPCIceCondenserContainmentMeltThrough

Tests a PClceCondenserContainment plant conditions model instance with properties:

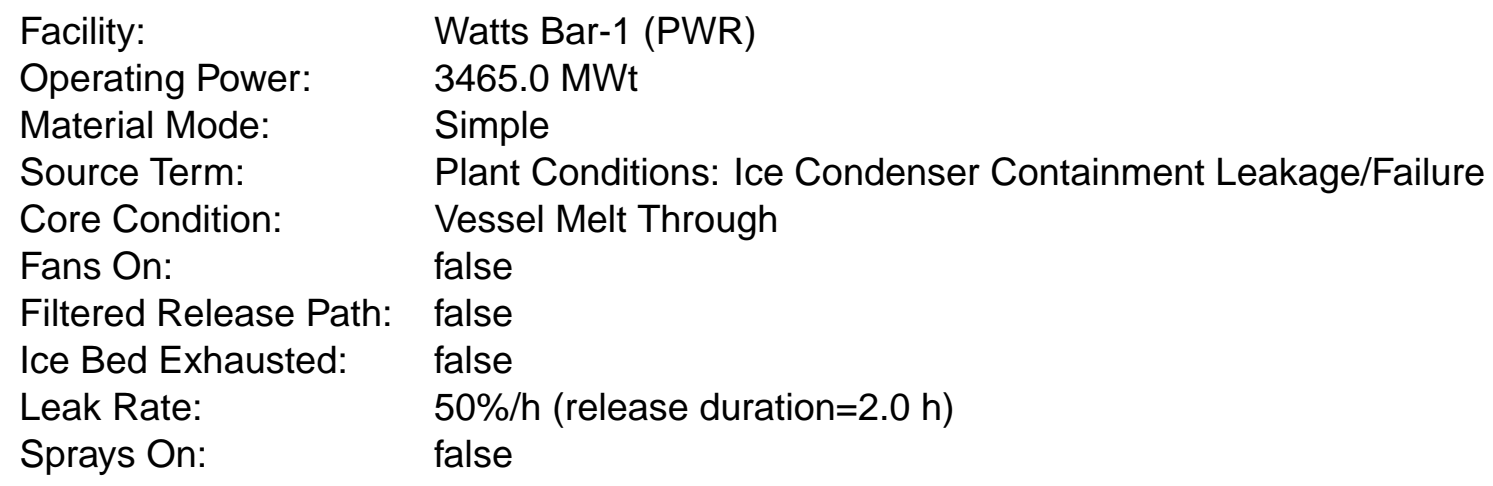

\section{Test: testPCLargeDrySubcontainmentLeakage}

Tests a PCLargeDrySubcontainmentLeakage plant conditions model instance with properties:

$\begin{array}{ll}\text { Facility: } & \text { Beaver Valley-1 (PWR) } \\ \text { Operating Power: } & 2733.0 \mathrm{MWt} \\ \text { Material Mode: } & \text { Simple } \\ \text { Source Term: } & \text { Plant Conditions: Large, Dry, or Subatmospheric Containment } \\ & \text { Leakage/Failure } \\ \text { Core Condition: } & \text { Gap Release } \\ \text { Filtered Release Path: } & \text { false } \\ \text { Leak Rate: } & 100 \% / \mathrm{h} \text { (release duration }=1.0 \mathrm{~h}) \\ \text { Sprays On: } & \text { false }\end{array}$




\section{Test: testPCPromptCriticalPowerExcursion}

Tests a PCPromptCriticalPowerExcursion plant conditions model instance with properties:

$\begin{array}{ll}\text { Facility: } & \text { Bilibino Unit A (RBMK) } \\ \text { Operating Power: } & \text { 33.0 MWt } \\ \text { Material Mode: } & \text { disabled } \\ \text { Source Term: } & \text { Plant Conditions: Prompt Critical Power Excursion } \\ \text { Core Involvement: } & \text { Total }\end{array}$

Test: testPCReproFacility

Tests a PCReproFacility plant conditions model instance with properties:

$\begin{array}{ll}\text { Facility: } & \begin{array}{l}\text { Savannah River (Reprocessing) } \\ \text { disabled } \\ \text { Material Mode: }\end{array} \\ \text { Source Term: } & \begin{array}{l}\text { Plant Conditions: Reprocessing Facility } \\ 1.0 \text { tonnes/day }\end{array} \\ \text { Plant Throughput: } & 100 \% / \mathrm{h} \text { (release duration=1.0 h) } \\ \text { Release Rate: } & \\ \text { Release Fraction by Component: } & 0.5 \\ \text { Aqueous Waste Treatment: } & 1.0 \\ \text { Dissolution: : } & 1.0 \\ \text { Feed Adjustment and Accountability: : } & 0.5 \\ \text { Pu Recovery: : } & 0.5 \\ \text { Solvent Treatment: : } & \\ \text { U-Pu Co-Decontamination, Partitioning, and } & \\ \text { U Purification: } & 1.0\end{array}$

\section{Test: testPCSodiumWaterReaction}

Tests a PCSodiumWaterReaction plant conditions model instance with properties:

$\begin{array}{ll}\text { Facility: } & \text { Phenix (LMFBR) } \\ \text { Custom Inventory: } & \text { stlauren_1.avc } \\ \text { Operating Power: } & \text { 699.0 MWt } \\ \text { Material Mode: } & \text { disabled } \\ \text { Source Term: } & \text { Plant Conditions: Sodium-Water Reaction } \\ \text { Core Condition: } & \text { Gap Release } \\ \text { Leak Rate: } & 50 \% / \mathrm{h} \text { (release duration=2.0 h) }\end{array}$

Test: testPCSteamTubeRuptureCoreDamage

Tests a PCSteamTubeRupture plant conditions model instance with properties: 
Facility:

Operating Power:

Material Mode:

Source Term:

Coolant Concentration:

Partitioned Generator:

Release Rate:

Release Source:
Dungeness B-1 (AGR)

1560.0 MWt

disabled

Plant Conditions: Steam Generator Tube Rupture (Coolant)

In-Vessel Severe Core Damage

false

1 Tube $(35 \% / h)$

Safety Valve

Test: testPCSteamTubeRuptureGapRelease

Tests a PCSteamTubeRupture plant conditions model instance with properties:

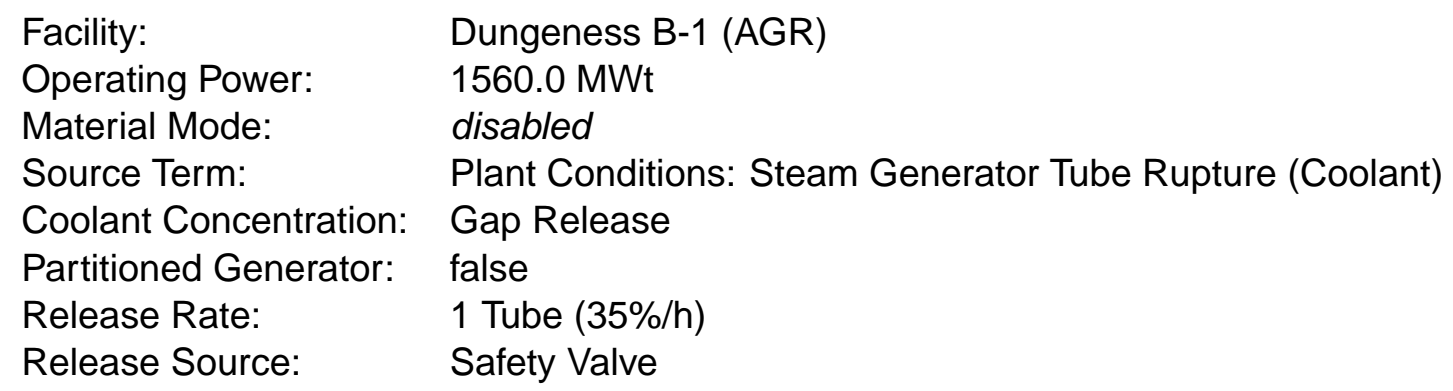

Test: testPCSubatmosphericConfinementLeakage

Tests a PCSubatmosphericConfinementLeakage plant conditions model instance with properties:

Facility:

Operating Power:

Material Mode:

Source Term:

Core Condition:

Leak Rate:

Pool Suppression System:
Rovno-1 (VVER-440/213)

1143.0 MWt

Simple

Plant Conditions: Subatmospheric Confinement Leakage/Failure

Gap Release

$100 \% / \mathrm{h}$ (release duration $=1.0 \mathrm{~h}$ )

Test: testPCWetWellLeakage

Tests a PCWetWellLeakage plant conditions model instance with properties: 
Facility:

Operating Power:

Material Mode:

Source Term:

Core Condition:

Leak Rate:

Filtered Release Path:

Wet Well:
Hamaoka-3 (BWR Mk-1)

3168.0 MWt

Simple

Plant Conditions: Wet Well Leakage/Failure (BWR Containment)

Gap Release

$100 \% / \mathrm{h}$ (release duration $=1.0 \mathrm{~h}$ )

false

Saturated

\section{Test: testRascalProject}

Tests a RascalProject model instance with properties:

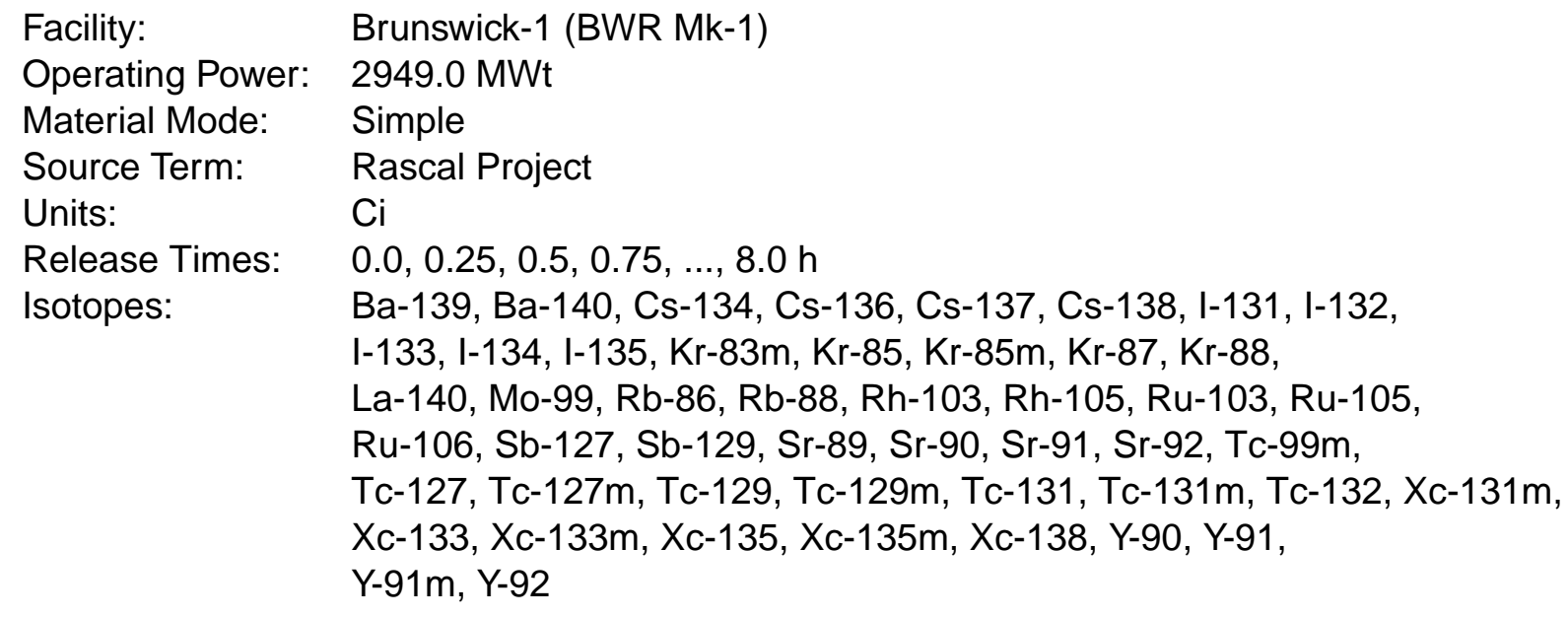

Test: testSevere

Tests an operational SevereModel instance with properties:

Facility: $\quad$ Brunswick-1 (BWR Mk-1)

Operating Power: $2949.0 \mathrm{MWt}$

Test: testSpentFuel

Tests an operational SpentFuel instance with properties:

Facility:

Operating Power:

Brunswick-1 (BWR Mk-1)

Source Term: 2949.0 MWt

Fuel Condition:

Spent Fuel/Spent Fuel Pool

Number of Batches:

Fuel Cladding Failure

Release Path:

1

Unfiltered

Sprays:

Off

Time Last Batch in Pool: 6 months prior to ReleaseToContainment 


\subsection{MODELTIMESTEST}

One of the most critical aspects of NFAC processing is correct management of event times within a scenario. There are six possible events whose times define or are defined by associated durations:

\section{Event}

Shutdown / Start of Decay

Release to Containment

Release to Environment

End of Release

End of Dispersion

End of Exposure

$\begin{array}{ll}\text { Associated Duration } & \text { From Event } \\ \text { Containment } & \text { Start of Decay } \\ \text { Shutdown } & \text { Start of Decay } \\ \text { Holdup } & \text { Release to Containment } \\ \text { Release } & \text { Release to Environment } \\ \text { Dispersion } & \text { End of Release } \\ \text { Exposure } & \text { End of Dispersion }\end{array}$

For some NFAC source models, individual events have no meaning and thus are ignored and not displayed in the GUI. Some times are computed directly from the model definition and thus are made uneditable (although displayed) in the GUI. Which times are displayed and/or editable are determined based on the model, and uneditable times must be computed based on the model parameters. Finally, uneditable times can be forced to be editable via the Advanced Mode toggle button in the When tab. The purpose of this test suite is to exercise event times management against various models and conditions. The eight tests are described below. Each unique times management class is tested.

NFAC components tested:

mil.dtra.hpac.models.nfac.CAcomp.data.ModelTimes

mil.dtra.hpac.models.nfac.CAcomp.data.times.AnalystMixTimesMgr

mil.dtra.hpac.models.nfac.CAcomp.data.times.IsotopicConcentrationsTimesMgr

mil.dtra.hpac.models.nfac.CAcomp.data.times.IsotopicReleaseRatesTimesMgr

mil.dtra.hpac.models.nfac.CAcomp.data.times.ModelTimesMgr

mil.dtra.hpac.models.nfac.CAcomp.data.times.ModelTimesMgrFactory

mil.dtra.hpac.models.nfac.CAcomp.data.times.OperationalTimesMgr

mil.dtra.hpac.models.nfac.CAcomp.data.times.PCContainmentByPassTimesMgr

mil.dtra.hpac.models.nfac.CAcomp.data.times.PercentInventoryTimesMgr

mil.dtra.hpac.models.nfac.CAcomp.data.times.RascalProjectTimesMgr

mil.dtra.hpac.models.nfac.CAcomp.data.times.SpentFuelTimesMgr

\section{Test: testAnalystMix}

This test verifies the following:

- The ModelTimesMgrFactory.getInstance() method correctly returns an AnalystMixTimesMgr instance for an AnalystMix model.

- The default times based on test input times are correctly set by the AnalystMixTimesMgr instance.

- After explicit settings of editable times, the Release to Environment and Release to Containment times are equivalent. 


\section{Test: testIsotopicConcentrations}

This test verifies the following:

- The ModelTimesMgrFactory.getInstance() method correctly returns an IsotopicConcentrationsTimesMgr instance for an IsotopicConcentrations model.

- The default times based on test input times are correctly set by the IsotopicConcentrationsTimesMgr instance.

- After explicit settings of editable times, the Start of Decay, Release to Containment, and Release to Environment times are equivalent.

\section{Test: testIsotopicReleaseRates}

This test verifies the following:

- The ModelTimesMgrFactory.getInstance() method correctly returns an IsotopicReleaseRatesTimesMgr instance for an IsotopicReleaseRates model.

- The default times based on test input times are correctly set by the IsotopicReleaseRatesTimesMgr instance.

- After explicit settings of editable times, the Start of Decay, Release to Containment, and Release to Environment times are equivalent.

\section{Test: testPCContainmentByPass}

This test verifies the following:

- The ModelTimesMgrFactory.getInstance() method correctly returns a PCContainmentByPassTimesMgr instance for an PCContainmentByPass model.

- The default times based on test input times are correctly set by the PCContainmentByPassTimesMgr instance.

- After explicit settings of editable times, the Start of Decay and Release to Containment times are equivalent, and the Shutdown, Release, Dispersion, and Exposure durations are all correct.

\section{Test: testPercentInventoryNoShutdown}

This test verifies the following:

- The ModelTimesMgrFactory.getInstance() method correctly returns a PercentInventoryTimesMgr instance for a PercentInventory model.

- After explicit setting of editable times, the Release to Containment and Release to Environment times are equivalent, the Shutdown Duration is zero, and the Release, Dispersion, and Exposure durations are correct.

- After explicit setting of editable times in lenient mode, the Release to Containment and Release to Environment times are equivalent, and the Shutdown, Release, Dispersion, and Exposure durations are correct for the input times. 


\section{Test: testPercentInventoryShutdown}

This test verifies the following:

- The ModelTimesMgrFactory.getInstance() method correctly returns a PercentInventoryTimesMgr instance for a PercentInventory model.

- After explicit setting of editable times, the Release to Containment and Release to Environment times are equivalent, and the Shutdown, Release, Dispersion, and Exposure durations are correct.

- After explicit setting of editable times in lenient mode, the Release to Containment and Release to Environment times are equivalent, and the Shutdown, Release, Dispersion, and Exposure durations are correct for the input times.

\section{Test: testRascalProject}

This test verifies the following:

- The ModelTimesMgrFactory.getInstance() method correctly returns a RascalProjectTimesMgr instance for an RascalProject model.

- After explicit setting of editable times, the Start of Decay, Release to Containment and Release to Environment times are equivalent.

\section{Test: testSpentFuel}

This test verifies the following:

- The ModelTimesMgrFactory.getInstance() method correctly returns a SpentFuelTimesMgr instance for an SpentFuel model.

- After explicit setting of editable times, the Start of Decay and Release to Containment times are equivalent, and the Holdup, Shutdown, Release, Dispersion, and Exposure durations are correct.

\subsection{PIRMODELDEFSTEST}

This suite contains 24 individual tests designed to verify proper function of Percent Inventory models and special processing associated with them. For each test there is a corresponding HPAC 6 project (.hpac6) file or a project export (.zip) file. For most tests a source term report $(. h t m l)$ is requested and compared to the baseline fixture, and when appropriate a log file $(. \log )$ is an additional fixture.

Included in this suite is verification that user overrides of model times and durations (see Section 4.3) result in correctly prorated PIR durations, and proper handling of material modes (refer to Section 2.2) and PIR release modes (refer to Section 2.3).

\section{Test: testPIRGroupsAvgNoShutdown}

Tests a PercentInventory model instance with no shutdown time and using groups material mode and average PIR release mode. 


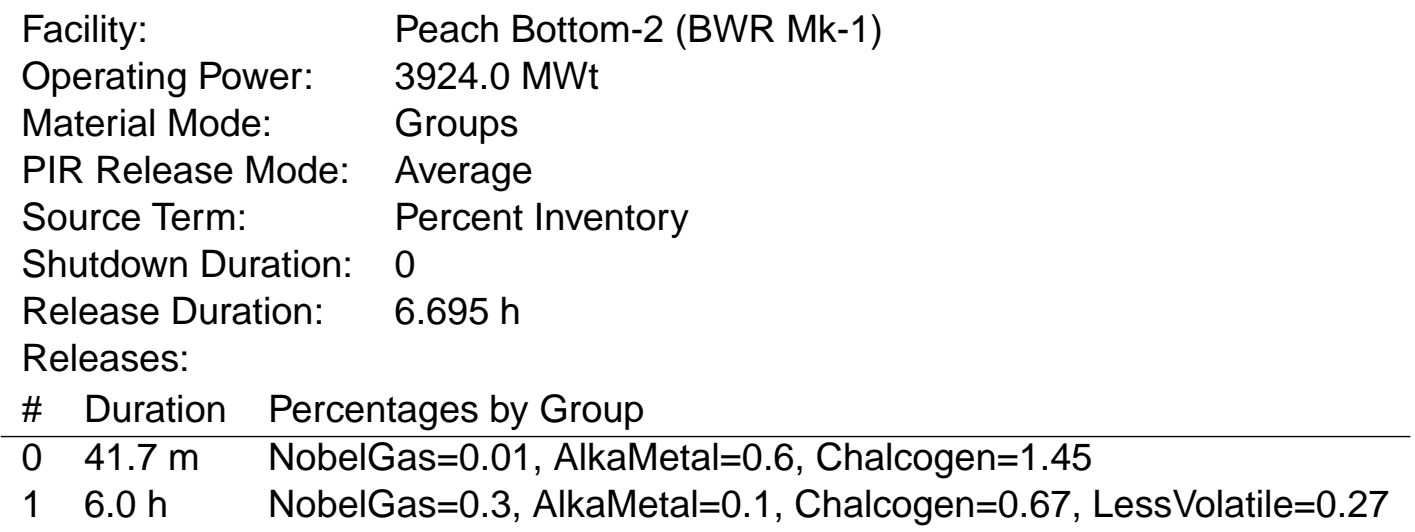

\section{Test: testPIRGroupsAvgWithShutdown}

Tests a PercentInventory model instance with a shutdown time and using groups material mode and average PIR release mode.

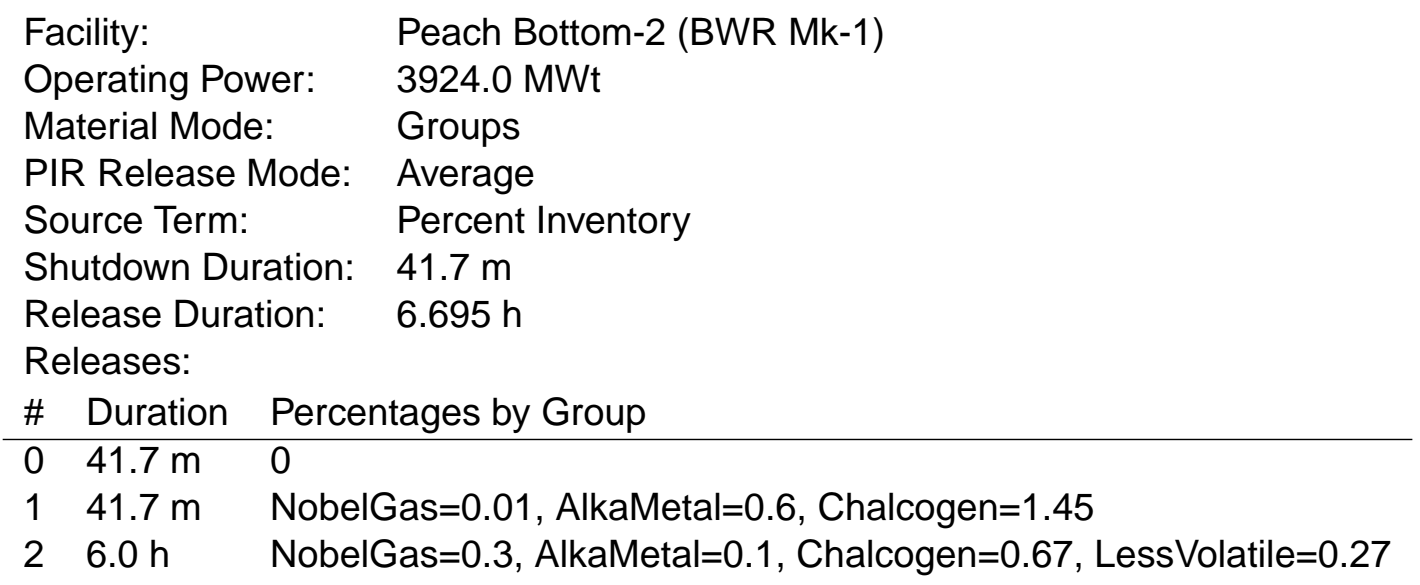

\section{Test: testPIRGroupsExplWithShutdown}

Tests a PercentInventory model instance with a shutdown time and using groups material mode and explicit PIR release mode.

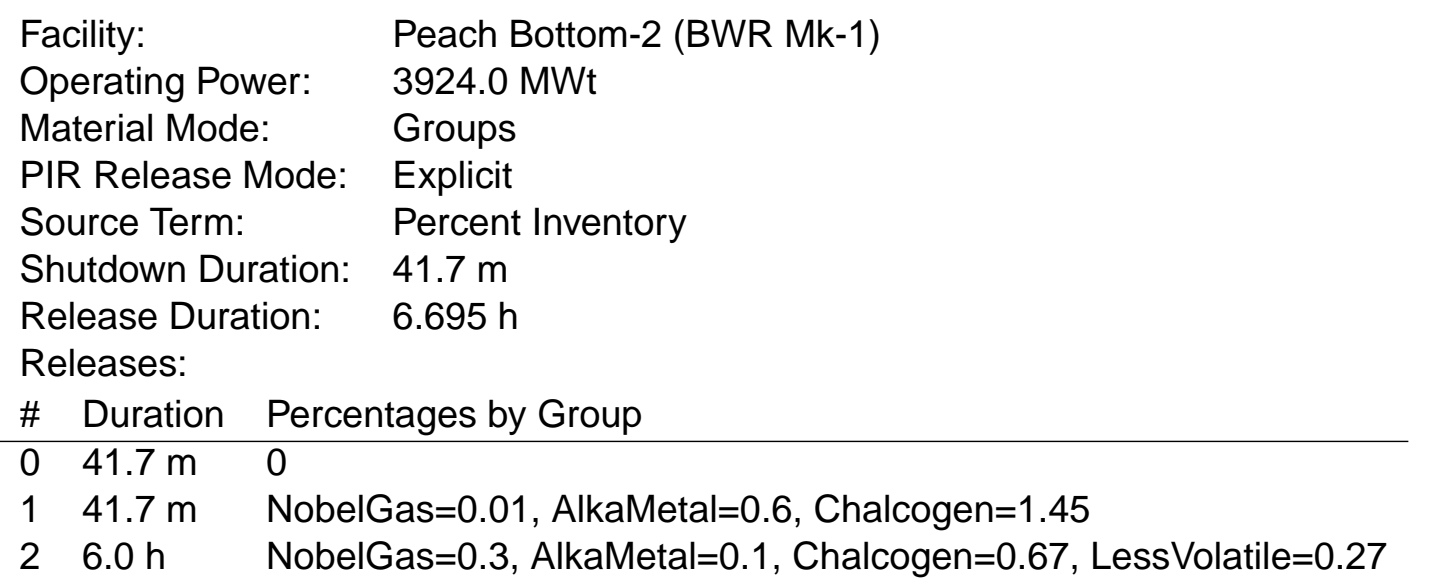




\section{Test: testPIRGroupsNoneNoShutdown}

Tests a Percentlnventory model instance with no shutdown time and using groups material mode and none PIR release mode.

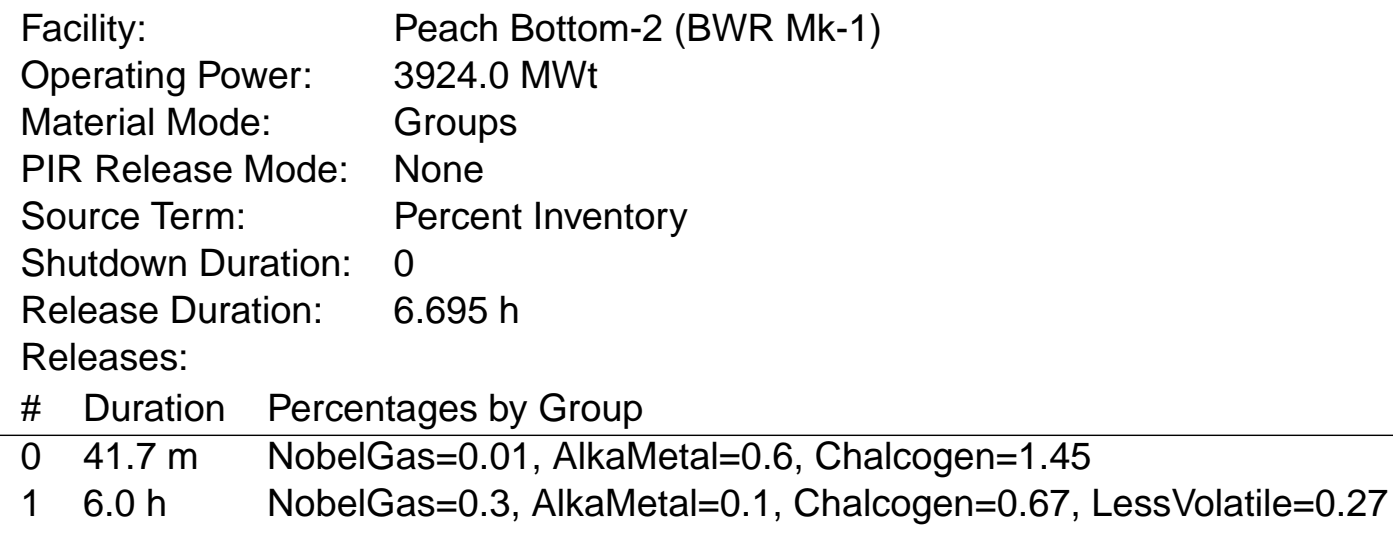

\section{Test: testPIRGroupsNoneWithShutdown}

Tests a PercentInventory model instance with a shutdown time and using groups material mode and none PIR release mode.

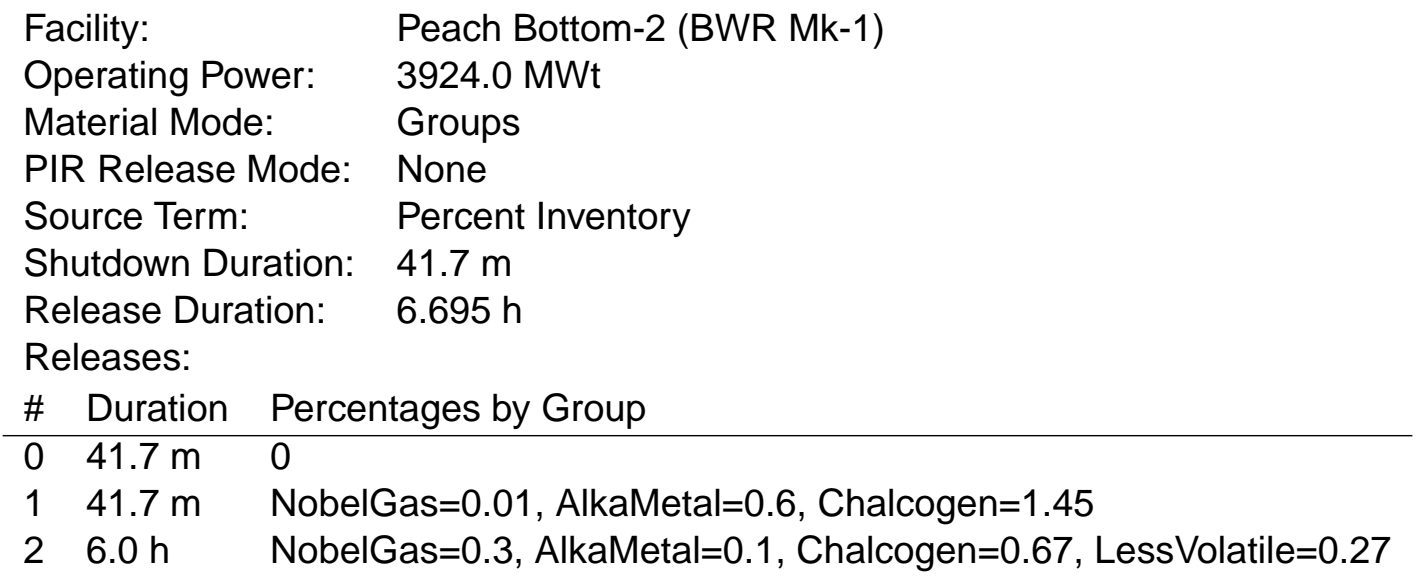

Test: testPIRMcAvgNoShutdown

Tests a PercentInventory model instance with no shutdown time and using multicomponent material mode and average PIR release mode. 


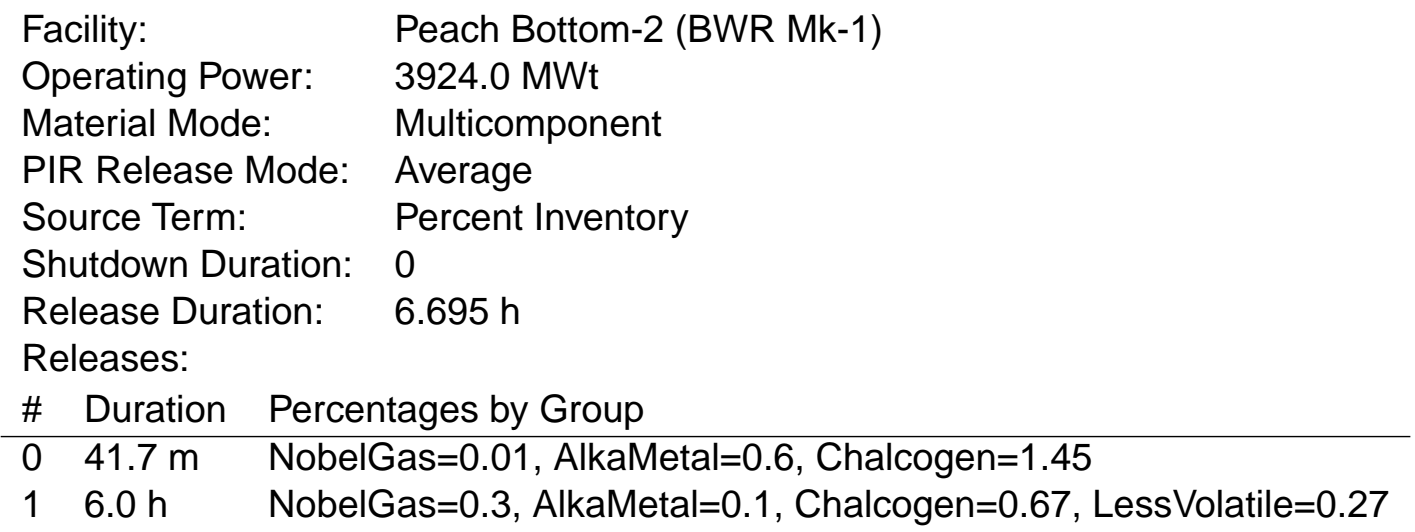

Test: testPIRMcAvgWithShutdown

Tests a PercentInventory model instance with a shutdown time and using multicomponent material mode and average PIR release mode.

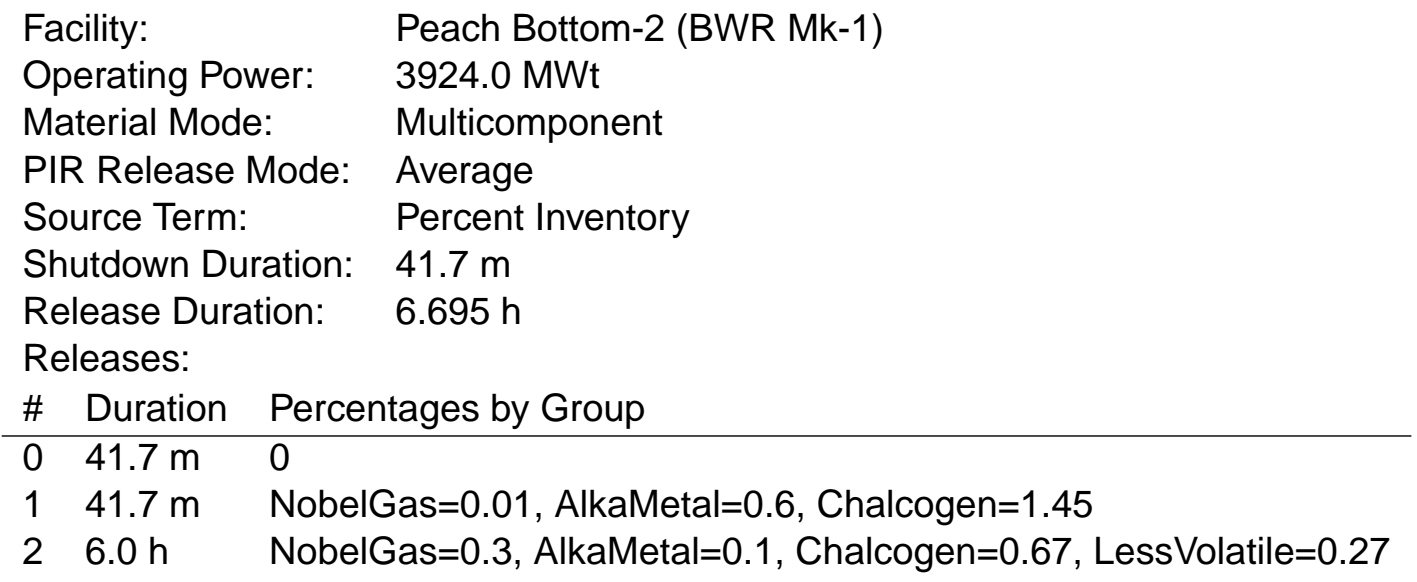

\section{Test: testPIRMcExplNoShutdown}

Tests a Percentlnventory model instance with no shutdown time and using multicomponent material mode and explicit PIR release mode.

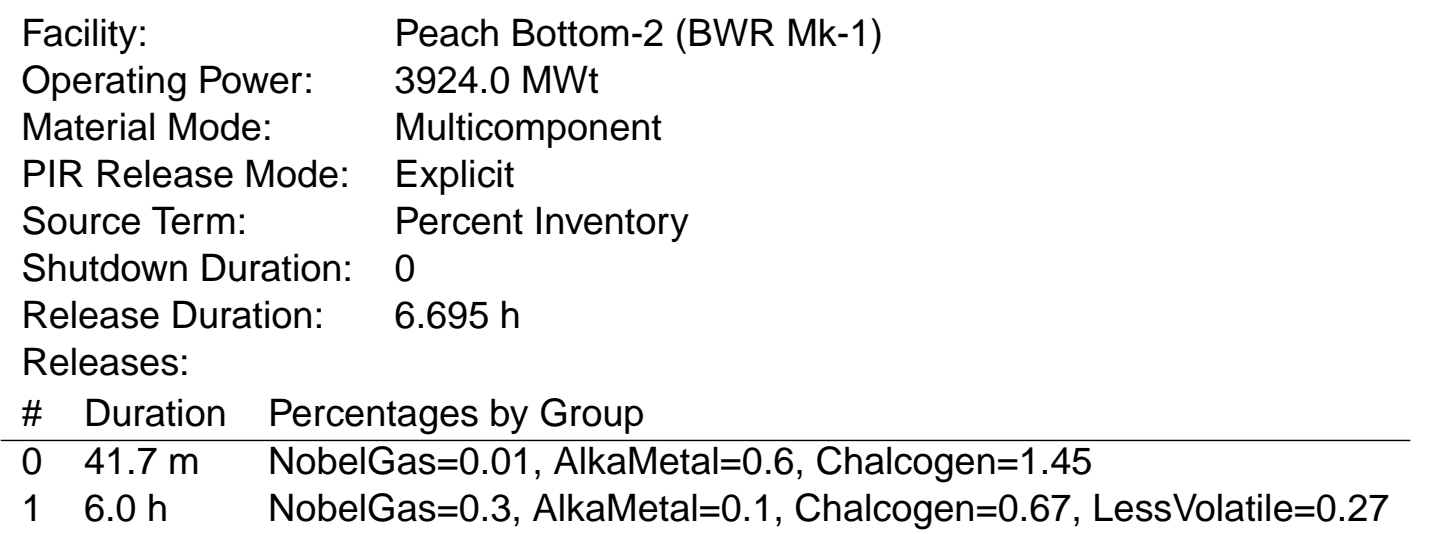




\section{Test: testPIRMcExplWithShutdown}

Tests a PercentInventory model instance with a shutdown time and using multicomponent material mode and explicit PIR release mode.

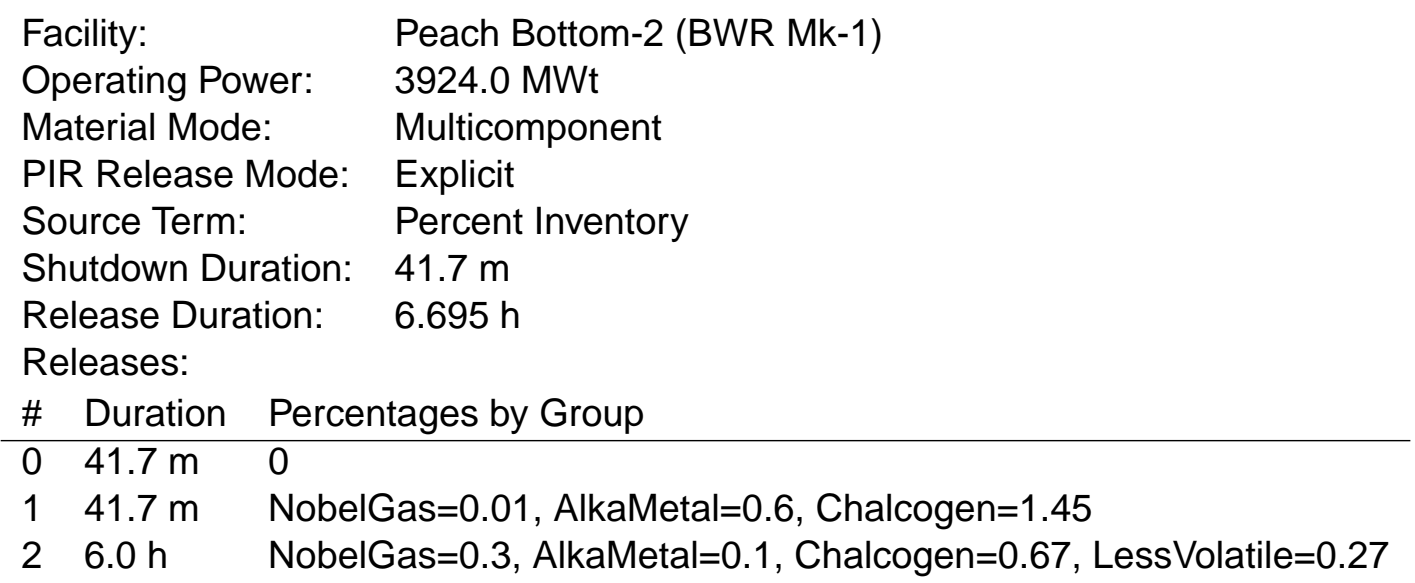

\section{Test: testPIRMcNoneNoShutdown}

Tests a PercentInventory model instance with no shutdown time and using multicomponent material mode and none PIR release mode.

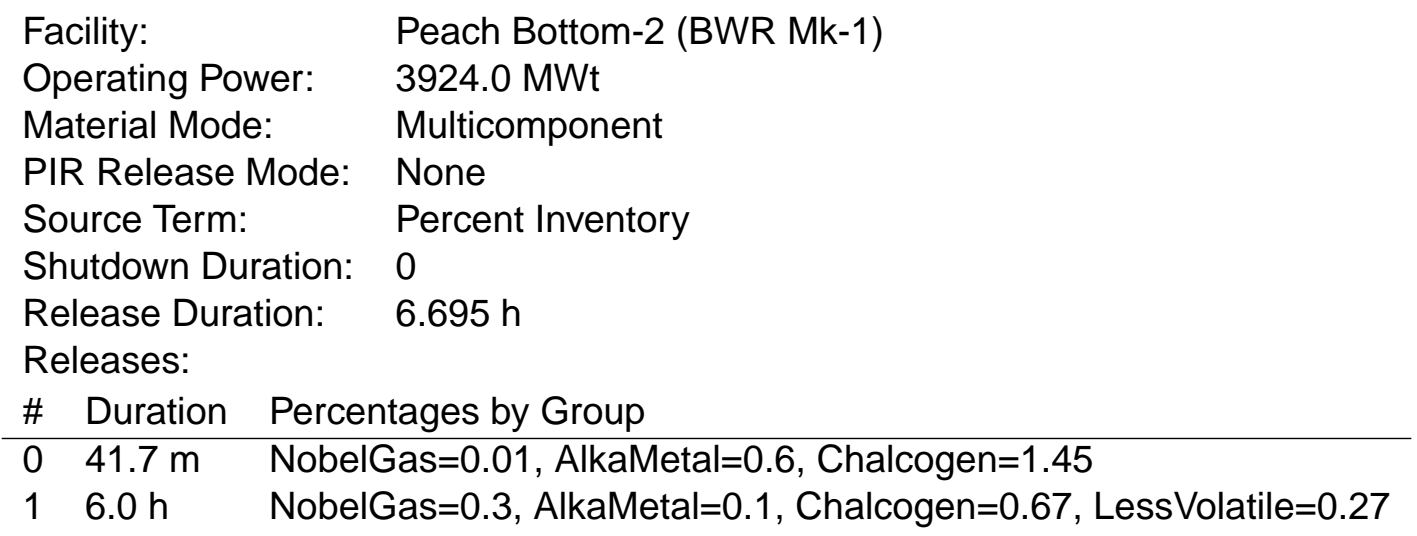

\section{Test: testPIRMcNoneWithShutdown}

Tests a PercentInventory model instance with a shutdown time and using multicomponent material mode and none PIR release mode. 


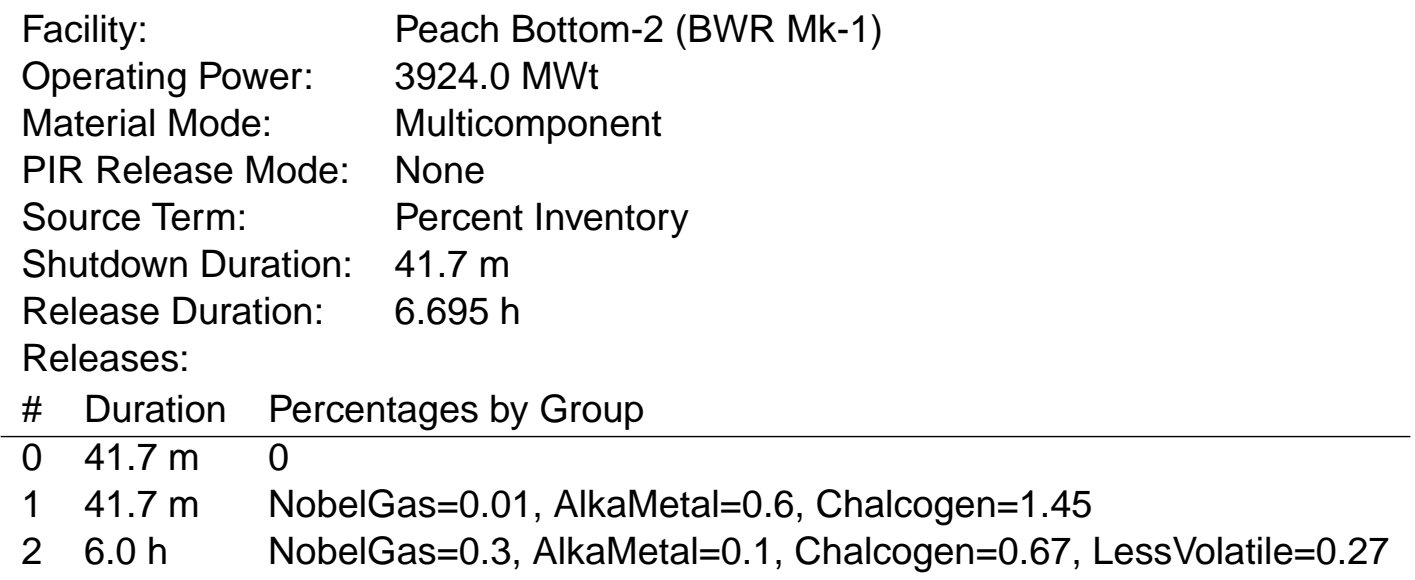

Test: testPIRSimpleAvgNoShutdown

Tests a PercentInventory model instance with no shutdown time and using simple material mode and average PIR release mode.

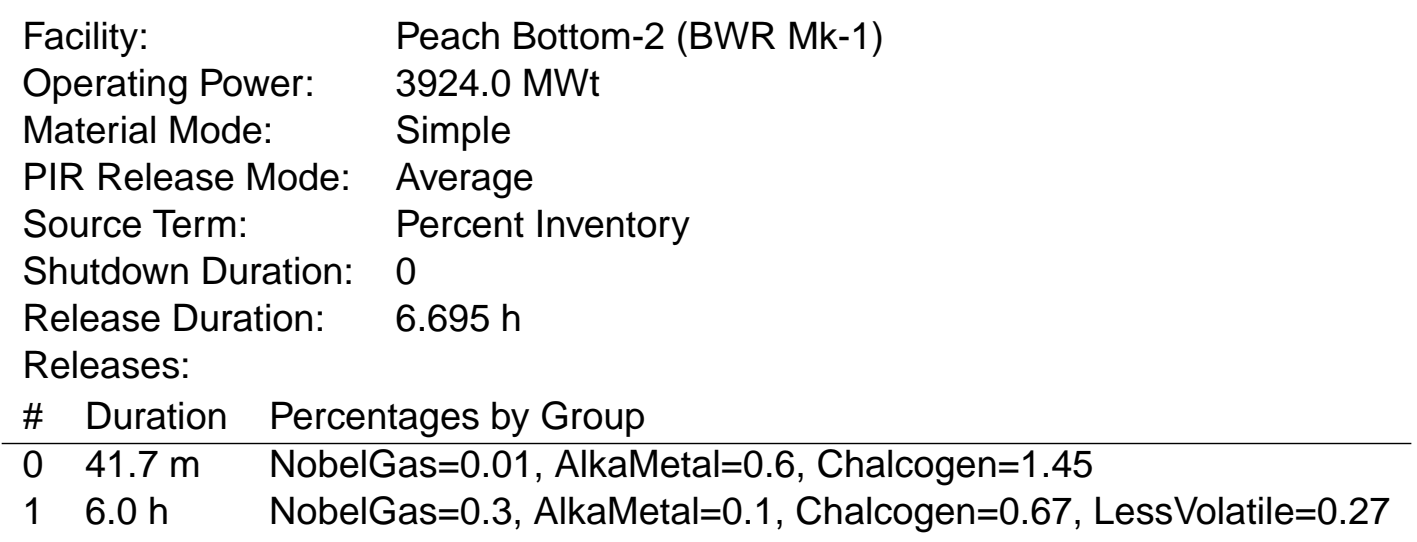

\section{Test: testPIRSimpleAvgWithShutdown}

Tests a PercentInventory model instance with a shutdown time and using simple material mode and average PIR release mode.

Facility:

Operating Power:

Peach Bottom-2 (BWR Mk-1)

Material Mode: 3924.0 MWt

PIR Release Mode: Average

Source Term: $\quad$ Percent Inventory

Shutdown Duration: $41.7 \mathrm{~m}$

Release Duration: $\quad 6.695 \mathrm{~h}$

Releases:

\begin{tabular}{lll} 
\# & Duration & Percentages by Group \\
\hline 0 & $41.7 \mathrm{~m}$ & 0 \\
1 & $41.7 \mathrm{~m}$ & NobelGas $=0.01$, AlkaMetal $=0.6$, Chalcogen $=1.45$ \\
2 & $6.0 \mathrm{~h}$ & NobelGas $=0.3$, AlkaMetal $=0.1$, Chalcogen $=0.67$, Less Volatile $=0.27$
\end{tabular}




\section{Test: testPIRSimpleExplNoShutdown}

Tests a Percentlnventory model instance with no shutdown time and using simple material mode and explicit PIR release mode.

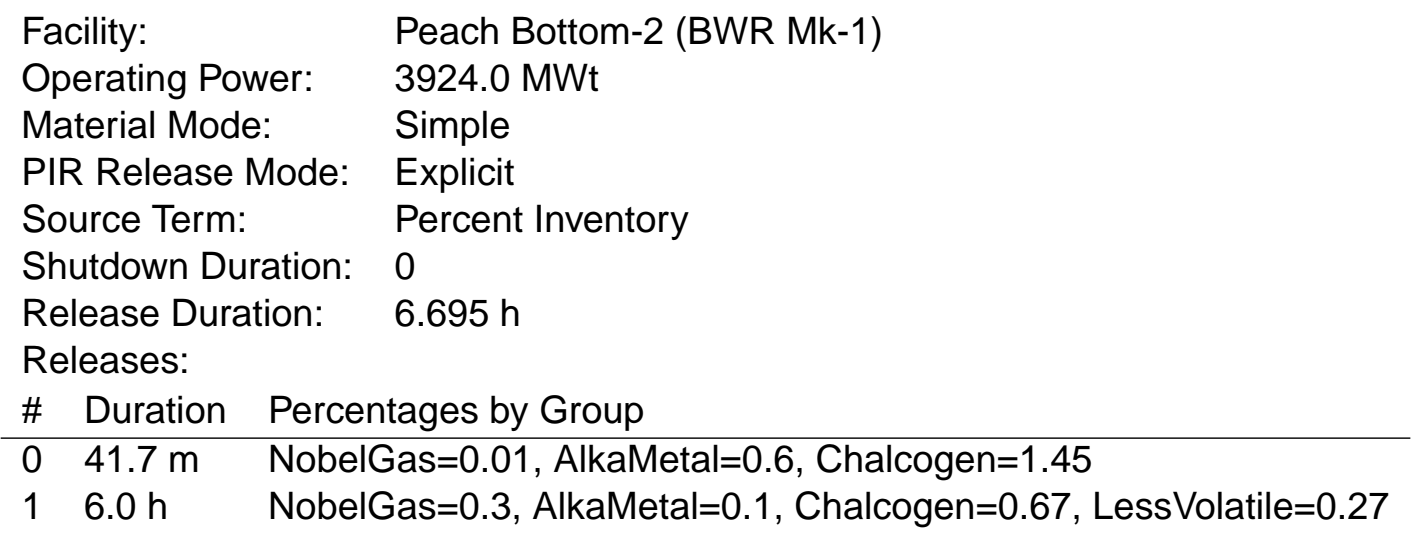

\section{Test: testPIRSimpleExplWithShutdown}

Tests a PercentInventory model instance with a shutdown time and using simple material mode and explicit PIR release mode.

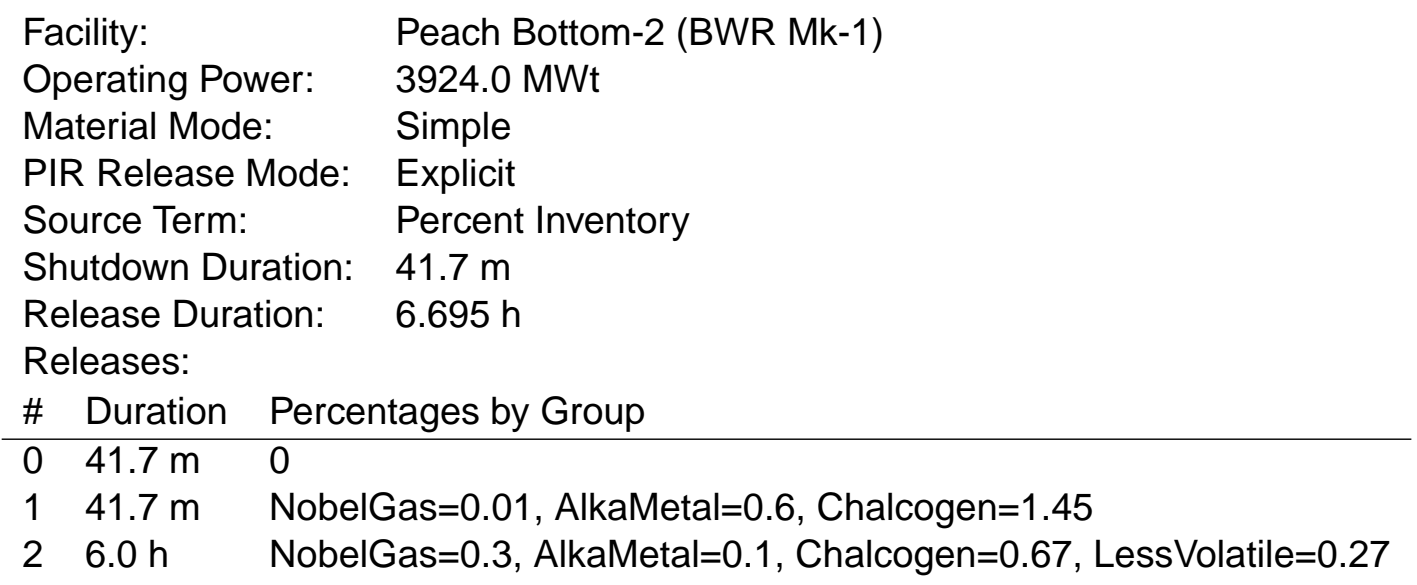

\section{Test: testPIRSimpleNoneNoShutdown}

Tests a PercentInventory model instance with no shutdown time and using simple material mode and none PIR release mode. 


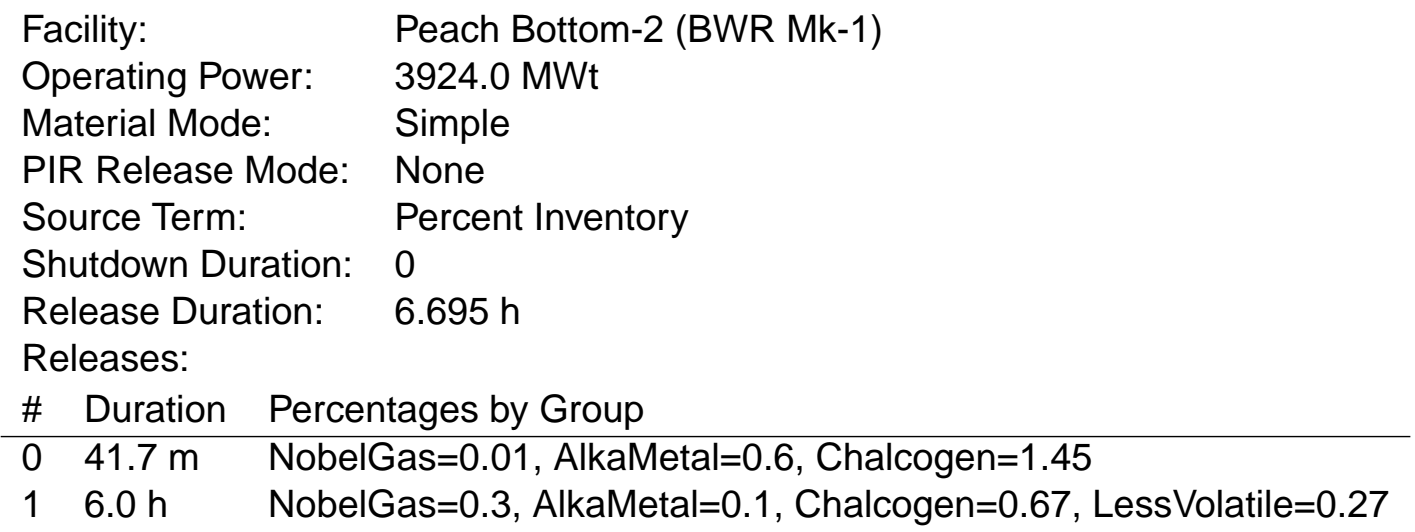

Test: testPIRSimpleNoneWithShutdown

Tests a Percentlnventory model instance with a shutdown time and using simple material mode and none PIR release mode.

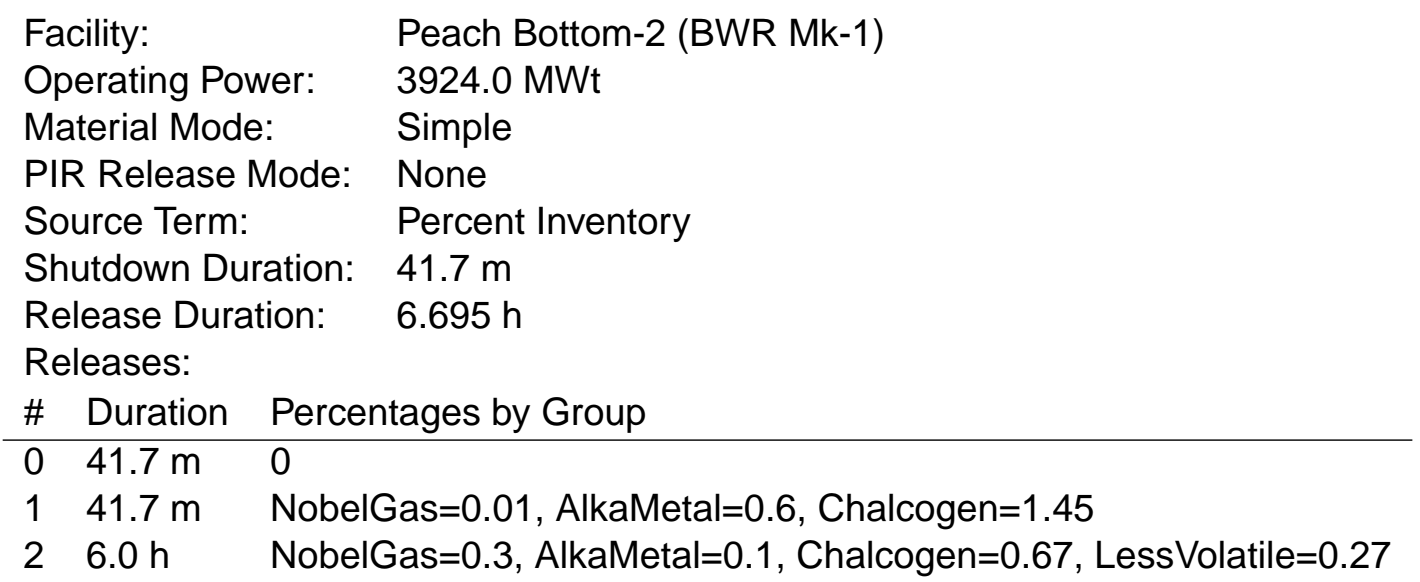

\section{Test: testPIRSubReleasesAverage}

Tests a PercentInventory model instance with the average PIR release mode.

\begin{tabular}{ll} 
Facility: & Peach Bottom-2 (BWR Mk-1) \\
Operating Power: & $3414.0 \mathrm{MWt}$ \\
Material Mode: & Simple \\
PIR Release Mode: & Average \\
Source Term: & Percent Inventory \\
Shutdown Duration: & $30.0 \mathrm{~m}$ \\
Release Duration: & $48.0 \mathrm{~h}$ \\
Releases: & \\
$\# \quad$ Duration & Percentages by Group \\
\hline 0 & $48.0 \mathrm{~h} \quad$ NobelGas $=100$
\end{tabular}




\section{Test: testPIRSubReleasesExplicit}

Tests a PercentInventory model instance with the explicit PIR release mode.

\begin{tabular}{ll} 
Facility: & Peach Bottom-2 (BWR Mk-1) \\
Operating Power: & $3414.0 \mathrm{MWt}$ \\
Material Mode: & Simple \\
PIR Release Mode: & Explicit \\
Source Term: & Percent Inventory \\
Shutdown Duration: & $30.0 \mathrm{~m}$ \\
Release Duration: & $48.0 \mathrm{~h}$ \\
Releases: & \\
$\# \quad$ Duration & Percentages by Group \\
\hline 0 & $48.0 \mathrm{~h} \quad$ NobelGas $=100$
\end{tabular}

Test: testPIRSubReleasesNone

Tests a Percentlnventory model instance with the none PIR release mode.

\begin{tabular}{ll} 
Facility: & Peach Bottom-2 (BWR Mk-1) \\
Operating Power: & $3414.0 \mathrm{MWt}$ \\
Material Mode: & Simple \\
PIR Release Mode: & None \\
Source Term: & Percent Inventory \\
Shutdown Duration: & $30.0 \mathrm{~m}$ \\
Release Duration: & $48.0 \mathrm{~h}$ \\
Releases: & \\
$\#$ Duration & Percentages by Group \\
\hline 0 & $48.0 \mathrm{~h} \quad$ NobelGas $=100$
\end{tabular}

Test: testPercentInventoryNoShutdownAsIs

Tests a PercentInventory model instance with no shutdown duration defined in the releases and no override of the model event times. 


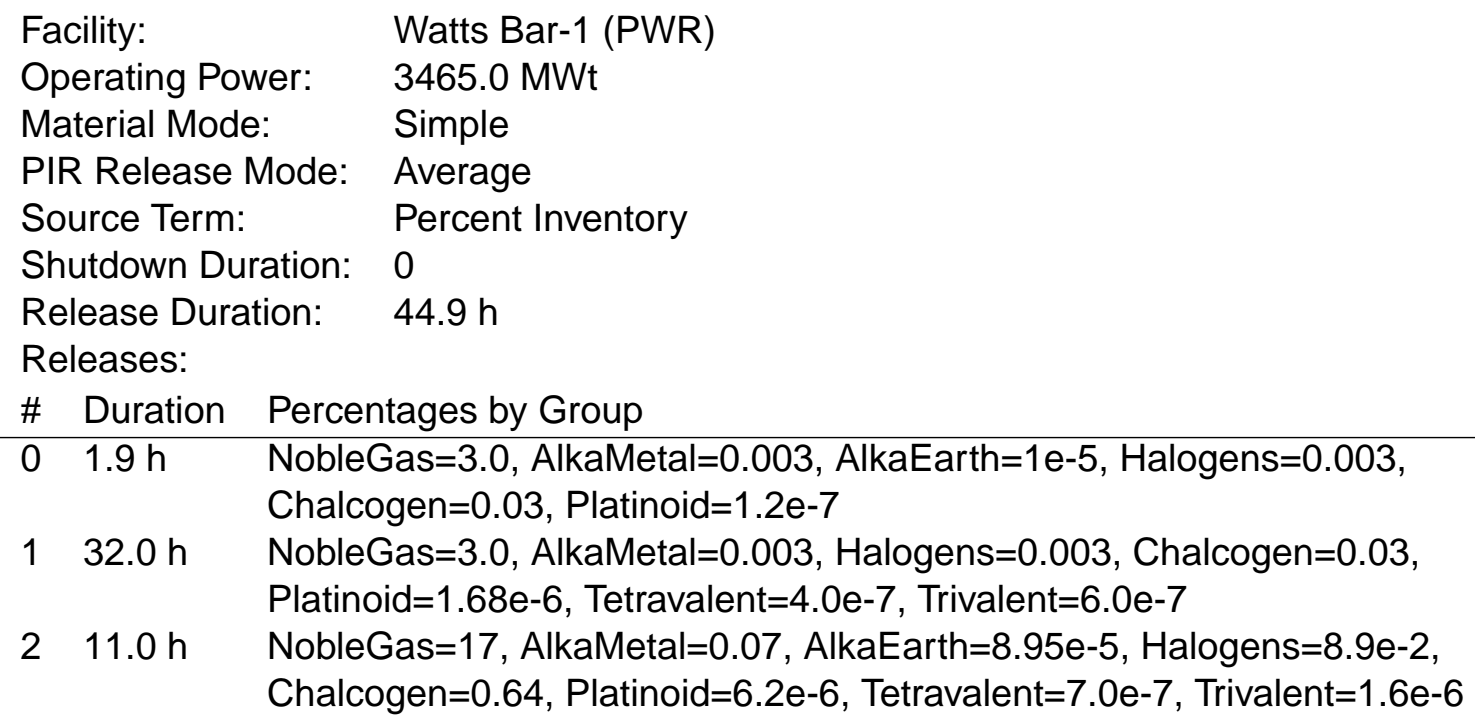

Test: testPercentInventoryNoShutdownForced

Tests a PercentInventory model instance with no shutdown duration defined in the releases, but model event times are overridden to force a shutdown time.

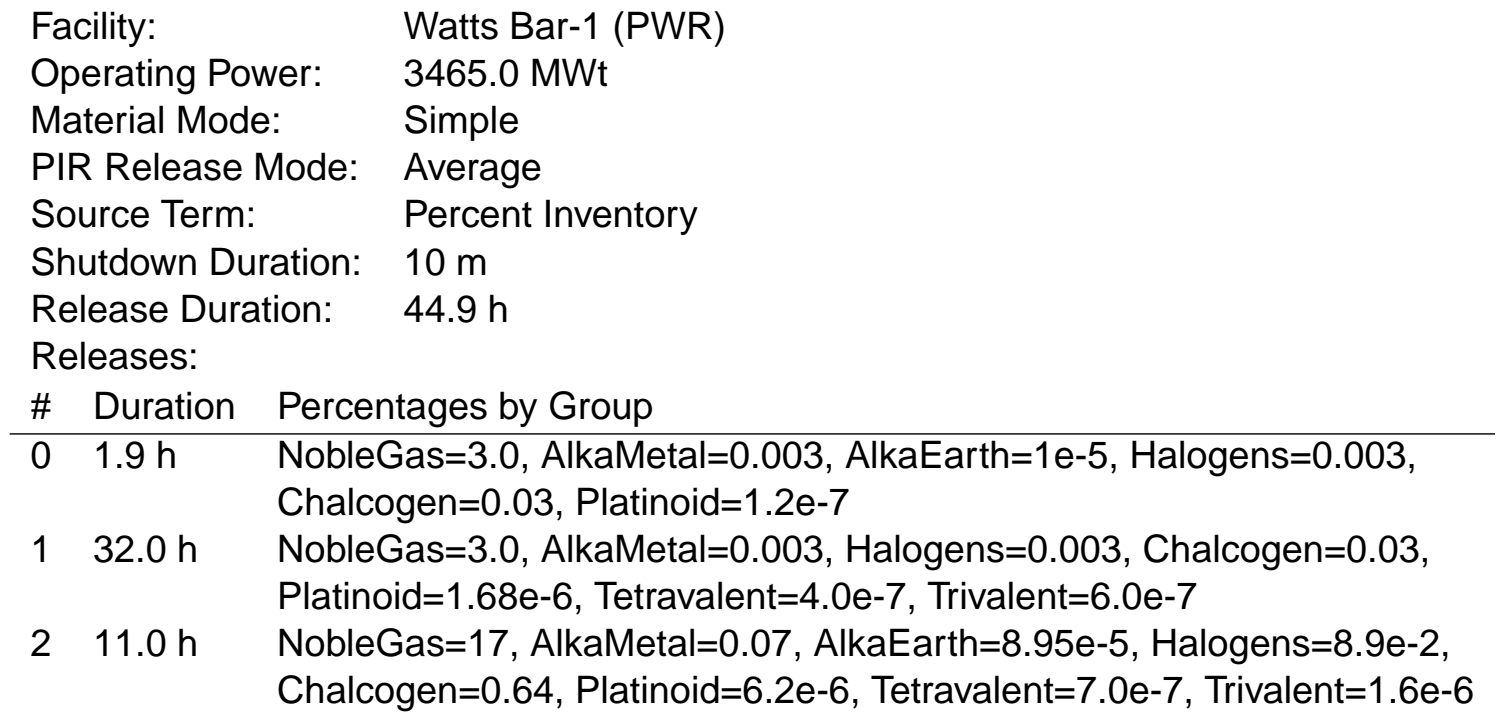

\section{Test: testPercentInventoryWithShutdownAsIs}

Tests a PercentInventory model instance with a shutdown duration defined in the releases and no override of the model event times. 


\begin{tabular}{|c|c|c|c|}
\hline \multicolumn{3}{|c|}{ Facility: } & Peach Bottom-2 (BWR Mk-1) \\
\hline \multicolumn{3}{|c|}{ Operating Power: } & 3414.0 MWt \\
\hline \multicolumn{3}{|c|}{ Material Mode: } & Simple \\
\hline \multicolumn{3}{|c|}{ PIR Release Mode: } & Average \\
\hline \multicolumn{3}{|c|}{ Source Term: } & Percent Inventory \\
\hline \multirow{2}{*}{\multicolumn{3}{|c|}{$\begin{array}{l}\text { Shutdown Duration: } \\
\text { Release Duration: }\end{array}$}} & $41.7 \mathrm{~m}$ \\
\hline & \multicolumn{3}{|c|}{ Releases: } \\
\hline \# & Duration & \multicolumn{2}{|c|}{ Percentages by Group } \\
\hline 0 & $41.7 \mathrm{~m}$ & \multicolumn{2}{|c|}{0} \\
\hline 1 & $41.7 \mathrm{~m}$ & \multicolumn{2}{|r|}{ NobelGas $=0.01$, AlkaMetal=0.6, Chalcogen $=0.35$} \\
\hline 2 & $83.33 \mathrm{~m}$ & \multicolumn{2}{|r|}{ NobelGas $=0.09$, AlkaMetal=1.7, AlkaEarth $=0.03$, Chalcogen $=1.45$} \\
\hline 3 & $250.0 \mathrm{~m}$ & \multicolumn{2}{|c|}{ NobelGas $=0.8$, AlkaMetal $=0.9$, Chalcogen $=0.45$} \\
\hline 4 & $303.0 \mathrm{~m}$ & \multicolumn{2}{|c|}{ NobelGas $=0.3$, AlkaMetal $=0.1$, Chalcogen $=0.22$, LessVolatile $=0.27$} \\
\hline
\end{tabular}

Test: testPercentInventoryWithShutdownForced

Tests a PercentInventory model instance with a shutdown duration defined in the releases, but model event times are overridden to force no shutdown time.

\begin{tabular}{|c|c|c|c|}
\hline \multicolumn{3}{|c|}{ Facility: } & Peach Bottom-2 (BWR Mk-1) \\
\hline \multicolumn{3}{|c|}{ Operating Power: } & 3414.0 MWt \\
\hline \multicolumn{3}{|c|}{ Material Mode: } & Simple \\
\hline \multicolumn{3}{|c|}{ PIR Release Mode: } & Average \\
\hline \multicolumn{3}{|c|}{ Source Term: } & Percent Inventory \\
\hline \multicolumn{3}{|c|}{ Shutdown Duration: } & $41.7 \mathrm{~m}$ \\
\hline \multicolumn{3}{|c|}{$\begin{array}{l}\text { Release Duration: } \\
\text { Releases: }\end{array}$} & $11.3 \mathrm{~h}$ \\
\hline \# & Duration & \multicolumn{2}{|c|}{ Percentages by Group } \\
\hline 0 & $41.7 \mathrm{~m}$ & \multicolumn{2}{|c|}{0} \\
\hline 1 & $41.7 \mathrm{~m}$ & \multicolumn{2}{|c|}{ NobelGas $=0.01$, AlkaMetal=0.6, Chalcogen $=0.35$} \\
\hline 2 & $83.33 \mathrm{~m}$ & \multicolumn{2}{|r|}{ NobelGas=0.09, AlkaMetal=1.7, AlkaEarth=0.03, Chalcogen $=1.45$} \\
\hline 3 & $250.0 \mathrm{~m}$ & \multicolumn{2}{|r|}{ NobelGas $=0.8$, AlkaMetal $=0.9$, Chalcogen $=0.45$} \\
\hline 4 & $303.0 \mathrm{~m}$ & \multicolumn{2}{|r|}{ NobelGas $=0.3$, AlkaMetal $=0.1$, Chalcogen $=0.22$, LessVolatile $=0.27$} \\
\hline
\end{tabular}

\subsection{PARTICLEGROUPSMGRTEST}

The major enhancement for NFAC version 6.3 was the addition of a new mode for processing materials. Refer to Section 2.2 for a description of the material modes. This test suite is comprised of seven tests that ensure the apportionment tables are properly read and activity apportionment and material creation are correctly processsed.

NFAC components tested:

mil.dtra.hpac.models.nfac.CAcomp.data.ParticleGroups

mil.dtra.hpac.models.nfac.CAcomp.data.ParticleGroupsMgr 


\section{Test: testBWRCounts}

Checks that the number of particle size groups, MELCOR groups, and table entries for each MELCOR group associated with the BWR facility type, as read from defaults.properties, are correct.

\section{Test: testBWRValues}

Checks that the BWR table entries giving apportionments for each MELCOR group and particle group, as read from defaults.properties are correct.

\section{Test: testPWRCounts}

Checks that the number of particle size groups, MELCOR groups, and table entries for each MELCOR group associated with the PWR facility type, as read from defaults.properties, are correct.

\section{Test: testPWRValues}

Checks that the PWR table entries giving apportionments for each MELCOR group and particle group, as read from defaults.properties, are correct.

\section{Test: testReadCounts}

Checks that the number of particle group tables and the list of associated facility types, as read from defaults.properties, are correct.

\section{Test: testTypeMap}

Verifies that the table associated with each reactor type and subtype are correct if the type/subtype should be supported and are not specified otherwise.

\section{Test: testWriteAndRead}

Verifies that the java.util.Properties entries as read from defaults.properties can be written and read back with the same resulting deserialized objects.

\subsection{SOURCETERMTABLETEST}

Another critical piece of NFAC functionality is determination of which of the 22 source models are valid or can be applied to a specific facility definition. A facility definition includes the facility selected from NFAC's facility "database" as well as a reference to any custom inventory file. NFAC's SourceTermTable and PlantConditionTables classes encapsulate the capability of determining available source terms. They are "data driven" in that they read the source_terms.data and plntcond.dat files, respectively, to drive the determination process.

This test suite includes 26 individual tests to verify that the source model availability process functions correctly.

NFAC components tested:

mil.dtra.hpac.models.nfac.CAcomp.data.Facility 
mil.dtra.hpac.models.nfac.CAcomp.data.FacilityDB

mil.dtra.hpac.models.nfac.CAcomp.data.FacilityDBMgr

mil.dtra.hpac.models.nfac.CAcomp.data.FacilityDef

mil.dtra.hpac.models.nfac.CAcomp.data.analyst.PlantConditionTables

mil.dtra.hpac.models.nfac.CAcomp.data.analyst.SourceTermTable

\section{Test: testAGRCompatibility}

Explicitly tests for individual model compatibility via the SourceTermTable.checkCompatability() method. The Dungeness B-1 and Windscale AGR facilities (without custom inventories) are tested against the following models with expected compatibility:

Dungeness B-1

$\begin{array}{ll}\text { AnalystMix } & \text { yes } \\ \text { ContainmentMonitorReading } & \text { no } \\ \text { IsotopicConcentrations } & \text { yes } \\ \text { IsotopicReleaseRates } & \text { yes } \\ \text { ModerateModel } & \text { no } \\ \text { PCLargeDrySubContainment } & \text { no } \\ \text { PercentInventory } & \text { yes } \\ \text { SevereModel } & \text { no } \\ \text { SpentFuel } & \text { no }\end{array}$

Windscale AGR

$\begin{array}{ll}\text { PCConfinementByPass } & \text { yes } \\ \text { PCConfinementLeakage } & \text { yes } \\ \text { PCContainmentByPass } & \text { no } \\ \text { PCContainmentLeakage } & \text { no } \\ \text { PCDryWellLeakage } & \text { no } \\ \text { PClceCondenserContainment } & \text { no } \\ \text { PCLargeDrySubContainmentLeakage } & \text { no } \\ \text { PCPromptCriticalPowerExcursion } & \text { no } \\ \text { PCReproFacility } & \text { no } \\ \text { PCSodiumWaterReaction } & \text { no } \\ \text { PCSteamTubeRupture } & \text { yes } \\ \text { PCSubatmosphericConfinementLeakage } & \text { no } \\ \text { PCWetWellLeakage } & \text { no }\end{array}$

\section{Test: testAGRSourceTerms}

Calls SourceTermTable.isOperationalSupported(), SourceTermTable.getFacilityTerms(), and PlantConditionTables.getPlantConditionTerms() with the following AGR facilities and conditions, verifying the correct source term names are returned: 


$\begin{array}{lll}\begin{array}{l}\text { With inventory }+ \text { Active } \\ \text { Dungeness B-1 }\end{array} & \text { With inventory }+ \text { Inactive } & \text { No inventory } \\ 1 \quad \text { Inventory filename set explicitly } & & \\ 2 \text { Inventerston B-1 } & \\ 2 \text { Inventory filename cleared explicitly } & \end{array}$

Test: testBWR0SourceTerms

Calls SourceTermTable.isOperationalSupported(), SourceTermTable.getFacilityTerms(), and PlantConditionTables.getPlantConditionTerms() with the following BWR type-0 facilities and conditions, verifying the correct source term names are returned:

$\frac{\text { With inventory }+ \text { Active }}{\text { Leibstadt }} \frac{\text { With inventory }+ \text { Inactive }}{\text { Brunsbuettel (kkb) }} \frac{\text { No inventory }}{\text { Leibstadt }^{2}}$

2 Inventory filename cleared explicitly

\section{Test: testBWR1SourceTerms}

Calls SourceTermTable.isOperationalSupported(), SourceTermTable.getFacilityTerms(), and PlantConditionTables.getPlantConditionTerms() with the following BWR type-1 facilities and conditions, verifying the correct source term names are returned:

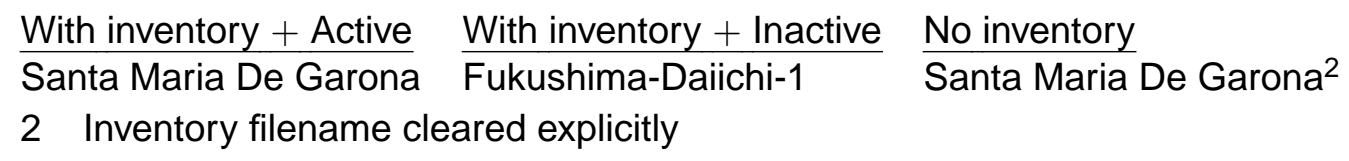

\section{Test: testBWR2SourceTerms}

Calls SourceTermTable.isOperationalSupported(), SourceTermTable.getFacilityTerms(), and PlantConditionTables.getPlantConditionTerms() with the following BWR type-2 facilities and conditions, verifying the correct source term names are returned:

\begin{tabular}{|c|c|c|}
\hline$\frac{\text { With inventory }+ \text { Active }}{\text { Fukushima-Daini-1 }}$ & $\frac{\text { With inventory }+ \text { Inactive }}{\text { Fukushima-Daini- } 1^{3}}$ & $\frac{\text { No inventory }}{\text { Fukushima-Daini- } 1^{2}}$ \\
\hline 2 Inventory filename cl & ared explicitly & \\
\hline
\end{tabular}

\section{Test: testBWR3SourceTerms}

Calls SourceTermTable.isOperationalSupported(), SourceTermTable.getFacilityTerms(), and PlantConditionTables.getPlantConditionTerms() with the following BWR type-3 facilities and conditions, verifying the correct source term names are returned:

$\frac{\text { With inventory }+ \text { Active }}{\text { Cofrentes }} \frac{\text { With inventory }+ \text { Inactive }}{\text { Cofrentes }^{3}} \frac{\text { No inventory }}{\text { Cofrentes }^{2}}$

2 Inventory filename cleared explicitly

3 Explicitly set inactive 


\section{Test: testFBRSourceTerms}

Calls SourceTermTable.isOperationalSupported(), SourceTermTable.getFacilityTerms(), and PlantConditionTables.getPlantConditionTerms() with the following FBR facilities and conditions, verifying the correct source term names are returned:

$\frac{\text { With inventory }+ \text { Active }}{\text { Monju }^{1}} \frac{\text { With inventory }+ \text { Inactive }}{\text { KNK II }} \frac{\text { No inventory }}{\text { Monju }}$

1 Inventory filename set explicitly

\section{Test: testGCHWRSourceTerms}

Calls SourceTermTable.isOperationalSupported(), SourceTermTable.getFacilityTerms(), and PlantConditionTables.getPlantConditionTerms() with the following GCHWR facilities and conditions, verifying the correct source term names are returned:

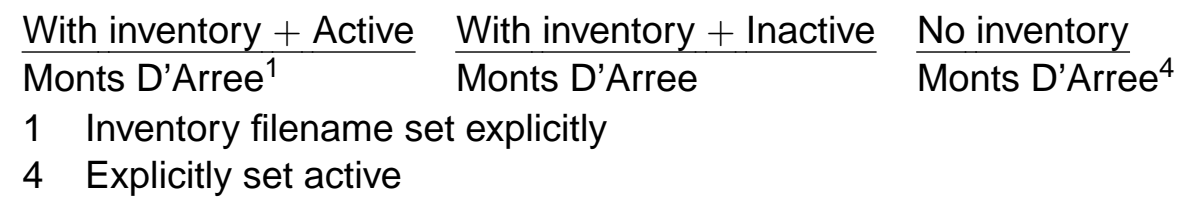

Test: testGCRSourceTerms

Calls SourceTermTable.isOperationalSupported(), SourceTermTable.getFacilityTerms(), and PlantConditionTables.getPlantConditionTerms() with the following GCR facilities and conditions, verifying the correct source term names are returned:

$$
\frac{\text { With inventory }+ \text { Active }}{\text { Oldbury-1 }} \frac{\text { With inventory }+ \text { Inactive }}{\text { Oldbury-2 }} \frac{\text { No inventory }}{\text { Latina }}
$$

Test: testHTGRSourceTerms

Calls SourceTermTable.isOperationalSupported(), SourceTermTable.getFacilityTerms(), and PlantConditionTables.getPlantConditionTerms() with the following HTGR facilities and conditions, verifying the correct source term names are returned:

\begin{tabular}{|c|c|c|}
\hline$\frac{\text { With inventory }+ \text { Active }}{\text { Peach Bottom- } 1^{1,4}}$ & $\frac{\text { With inventory }+ \text { Inactive }}{\text { Peach Bottom }-1^{1}}$ & $\frac{\text { No inventory }}{\text { Peach Bottom-1 }}$ \\
\hline 1 Inventory filename s & t explicitly & \\
\hline Explicitly set active & & \\
\hline
\end{tabular}

Test: testHWGCRSourceTerms

Calls SourceTermTable.isOperationalSupported(), SourceTermTable.getFacilityTerms(), and PlantConditionTables.getPlantConditionTerms() with the following HWGCR facilities and conditions, verifying the correct source term names are returned: 


\begin{tabular}{|c|c|c|}
\hline $\begin{array}{l}\text { With inventory }+ \text { Active } \\
\text { A-1 Bohunice }{ }^{1,4}\end{array}$ & $\frac{\text { With inventory }+ \text { Inactive }}{A-1 \text { Bohunice }^{1}}$ & $\frac{\text { No inventory }}{\text { A-1 Bohunice }}$ \\
\hline 1 Inventory filename s & t explicitly & \\
\hline Explicitly set active & & \\
\hline
\end{tabular}

\section{Test: testHWLWRSourceTerms}

Calls SourceTermTable.isOperationalSupported(), SourceTermTable.getFacilityTerms(), and PlantConditionTables.getPlantConditionTerms() with the following HWLWR facilities and conditions, verifying the correct source term names are returned:

\begin{tabular}{|c|c|c|}
\hline With inventory + Active & With inventory + Inactive & No inventory \\
\hline$\overline{\text { Fugen ATR }^{1,4}}$ & $\overline{\text { Fugen ATR }^{1}}$ & Fugen ATR \\
\hline 1 Inventory filename s & t explicitly & \\
\hline
\end{tabular}

\section{Test: testLGRSourceTerms}

Calls SourceTermTable.isOperationalSupported(), SourceTermTable.getFacilityTerms(), and PlantConditionTables.getPlantConditionTerms() with the following LGR facilities and conditions, verifying the correct source term names are returned:

\begin{tabular}{|c|c|c|}
\hline With inventory + Active & With inventory + Inactive & No inventory \\
\hline$\overline{\text { Hanford }-\mathrm{N}^{1,4}}$ & Toitsk E & Troitsk F \\
\hline Inventory filename s & t explicitly & \\
\hline Explicitly set active & & \\
\hline
\end{tabular}

\section{Test: testLMFBRSourceTerms}

Calls SourceTermTable.isOperationalSupported(), SourceTermTable.getFacilityTerms(), and PlantConditionTables.getPlantConditionTerms() with the following LMFBR facilities and conditions, verifying the correct source term names are returned:
With inventory + Active With inventory + Inactive No inventory
Beloyarsky-3(BN-600) ${ }^{1} \quad$ Beloyarsky-4(BN-800) ${ }^{1,3} \quad$ Beloyarsky-3(BN-600)
1 Inventory filename set explicitly
3 Explicitly set inactive

\section{Test: testLWRSourceTerms}

Calls SourceTermTable.isOperationalSupported(), SourceTermTable.getFacilityTerms(), and PlantConditionTables.getPlantConditionTerms() with the following LWR facilities and conditions, verifying the correct source term names are returned:

\begin{tabular}{|c|c|c|}
\hline $\begin{array}{l}\text { With inventory + Active } \\
\text { Generic LWR Site }\end{array}$ & $\frac{\text { With inventory }+ \text { Inactive }}{\text { Generic LWR Site }^{3}}$ & $\frac{\text { No inventory }}{\text { Generic LWR Site }}$ \\
\hline Inventory filename cl & ared explicitly & \\
\hline Explicitly set inactive & & \\
\hline
\end{tabular}




\section{Test: testPHWRSourceTerms}

Calls SourceTermTable.isOperationalSupported(), SourceTermTable.getFacilityTerms(), and PlantConditionTables.getPlantConditionTerms() with the following PHWR facilities and conditions, verifying the correct source term names are returned:

$$
\frac{\text { With inventory }+ \text { Active }}{\text { Bruce-1 }} \frac{\text { With inventory }+ \text { Inactive }}{\text { Gentilly-1 }} \frac{\text { No inventory }}{\text { Agesta }}
$$

\section{Test: testPWRSourceTerms}

Calls SourceTermTable.isOperationalSupported(), SourceTermTable.getFacilityTerms(), and PlantConditionTables.getPlantConditionTerms() with the following PWR facilities and conditions, verifying the correct source term names are returned:

$$
\frac{\text { With inventory }+ \text { Active }}{\text { Doel-1 }} \frac{\text { With inventory }+ \text { Inactive }}{\text { BR-3 }} \frac{\text { No inventory }}{\text { Doel- } 2^{2}}
$$

2 Inventory filename cleared explicitly

\section{Test: testRBMKSourceTerms}

Calls SourceTermTable.isOperationalSupported(), SourceTermTable.getFacilityTerms(), and PlantConditionTables.getPlantConditionTerms() with the following RBMK facilities and conditions, verifying the correct source term names are returned:

$\begin{array}{lll}\text { With inventory }+ \text { Active } & \text { With inventory }+ \text { Inactive } & \text { No inventory } \\ \text { Bilibino Unit A } & \text { Bilibino Unit A }{ }^{2}\end{array}$

2 Inventory filename cleared explicitly

\section{Test: testReproSourceTerms}

Calls SourceTermTable.isOperationalSupported(), SourceTermTable.getFacilityTerms(), and PlantConditionTables.getPlantConditionTerms() with the following Repro facilities and conditions, verifying the correct source term names are returned:

$\frac{\text { With inventory }+ \text { Active }}{\text { Idaho Falls ICPP }}$ With inventory + Inactive
2 Inventory filename cleared explicitly

\section{Test: testResearchSourceTerms}

Calls SourceTermTable.isOperationalSupported(), SourceTermTable.getFacilityTerms(), and PlantConditionTables.getPlantConditionTerms () with the following Research facilities and conditions, verifying the correct source term names are returned:

$$
\frac{\text { With inventory }+ \text { Active }}{\text { AFRRI TRIGA Reactor }} \quad \begin{array}{ll}
\text { With inventory }+ \text { Inactive } & \text { No inventory } \\
\text { ATR } & \text { Bol }
\end{array}
$$




\section{Test: testSGHWRSourceTerms}

Calls SourceTermTable.isOperationalSupported(), SourceTermTable.getFacilityTerms(), and PlantConditionTables.getPlantConditionTerms() with the following SGHWR facilities and conditions, verifying the correct source term names are returned:

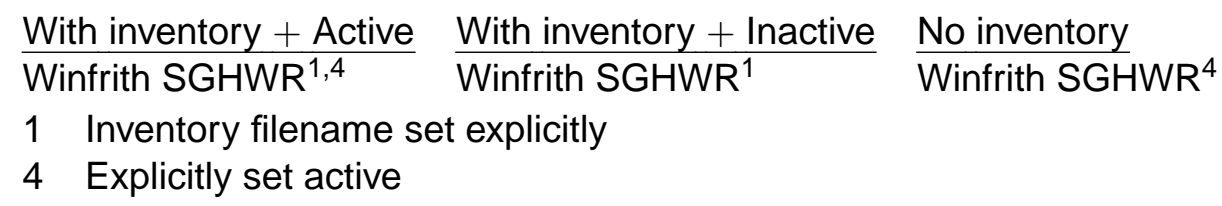

\section{Test: testSPCR6787}

This test verifies changes to research reactors in the NFAC facility database resulting from SPCR 6787. It calls FacilityDB.getFacilities() to list all facilities for a country code and then searches the list to the match name and research reactor facility type. Explicit tests by country are as follows.

\begin{tabular}{ll} 
Country & Facilities \\
\hline Albania & (no existing research reactors) \\
China & CARR (Beijing), CEFR (Beijing), CMRR (Mienyang), HTR-10 (Beijing), HWRR-II (Beijing), \\
North Korea & IRT-DPRK, YONGBYON GCR \\
Portugal & RPI
\end{tabular}

\section{Test: testVVER0SourceTerms}

Calls SourceTermTable.isOperationalSupported(), SourceTermTable.getFacilityTerms(), and PlantConditionTables.getPlantConditionTerms() with the following VVER type-0 facilities and conditions, verifying the correct source term names are returned:

$\frac{\text { With inventory }+ \text { Active }}{\text { Bushehr-1 }} \frac{\text { With inventory }+ \text { Inactive }}{\text { Bushehr-1 }} \frac{\text { No inventory }}{\text { Bushehr-12 }}$
2 Inventory filename cleared explicitly
3 Explicitly set inactive

\section{Test: testVVER1SourceTerms}

Calls SourceTermTable.isOperationalSupported(), SourceTermTable.getFacilityTerms(), and PlantConditionTables.getPlantConditionTerms() with the following VVER type-1 facilities and conditions, verifying the correct source term names are returned:

$\frac{\text { With inventory }+ \text { Active }}{\text { Metzamor-2 }} \frac{\text { With inventory }+ \text { Inactive }}{\text { Metzamor-1 }} \frac{\text { No inventory }}{\text { Kola- }{ }^{2}}$

2 Inventory filename cleared explicitly 


\section{Test: testVVER2SourceTerms}

Calls SourceTermTable.isOperationalSupported(), SourceTermTable.getFacilityTerms(), and PlantConditionTables.getPlantConditionTerms() with the following VVER type-2 facilities and conditions, verifying the correct source term names are returned:

$\begin{array}{lll}\frac{\text { With inventory }+ \text { Active }}{\text { Dukovany- } 1} & \text { With inventory }+ \text { Inactive } & \text { No inventory } \\ 2 \text { Inventory filename cleared explicitly } & & \text { Dukovany-2 } 2^{2}\end{array}$

\section{Test: testVVER3SourceTerms}

Calls SourceTermTable.isOperationalSupported(), SourceTermTable.getFacilityTerms(), and PlantConditionTables.getPlantConditionTerms() with the following VVER type-3 facilities and conditions, verifying the correct source term names are returned:

\begin{tabular}{|c|c|c|}
\hline With inventory + Active & $\frac{\text { With inventory }+ \text { Inactive }}{K}$ & $\frac{\text { No inventory }}{\text { Kalinin-32 }}$ \\
\hline Inventory filena & ared explicitly & \\
\hline Explicitly set inactive & & \\
\hline
\end{tabular}

\subsection{SOURCETERMTABLETEST2}

This test suite is distinguished from SourceTermTableTest for cases in which no plant condition models apply. For now, this is limited to the new HWRESRC research facility type.

\section{Test: testHWRESRC3SourceTerms}

Calls SourceTermTable.isOperationalSupported(), SourceTermTable.getFacilityTerms(), and PlantConditionTables.getPlantConditionTerms() with the following HWRESRC facilities and conditions, verifying the correct source term names are returned:

$$
\frac{\text { With inventory }+ \text { Active }}{\mathrm{IR}-40} \frac{\text { No inventory }}{\mathrm{IR}-40^{2}} 2 \text { Inventory filename cleared explicitly }
$$

\subsection{RTHTIMESTEST}

Interacts with RTH to test times returned from decay calculations when various decay times are passed as input. 


\begin{tabular}{|c|c|c|}
\hline Test Iteration & Start Time & Offsets \\
\hline 0 & $\overline{3.0 \mathrm{~h}}$ & 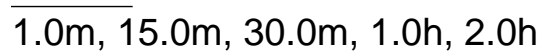 \\
\hline 1 & $6.0 \mathrm{~h}$ & $1.0 \mathrm{~m}, 15.0 \mathrm{~m}, 30.0 \mathrm{~m}, 1.0 \mathrm{~h}, 2.0 \mathrm{~h}$ \\
\hline 2 & $12.0 \mathrm{~h}$ & $1.0 \mathrm{~m}, 15.0 \mathrm{~m}, 30.0 \mathrm{~m}, 1.0 \mathrm{~h}, 2.0 \mathrm{~h}$ \\
\hline 3 & 24.0h & $1.0 \mathrm{~m}, 15.0 \mathrm{~m}, 30.0 \mathrm{~m}, 1.0 \mathrm{~h}, 2.0 \mathrm{~h}$ \\
\hline 4 & 48.0h & $1.0 \mathrm{~m}, 15.0 \mathrm{~m}, 30.0 \mathrm{~m}, 1.0 \mathrm{~h}, 2.0 \mathrm{~h}$ \\
\hline 5 & $96.0 \mathrm{~h}$ & $1.0 \mathrm{~m}, 15.0 \mathrm{~m}, 30.0 \mathrm{~m}, 1.0 \mathrm{~h}, 2.0 \mathrm{~h}$ \\
\hline 6 & 192.0h & $1.0 \mathrm{~m}, 15.0 \mathrm{~m}, 30.0 \mathrm{~m}, 1.0 \mathrm{~h}, 2.0 \mathrm{~h}$ \\
\hline 7 & 384.0h & $1.0 \mathrm{~m}, 15.0 \mathrm{~m}, 30.0 \mathrm{~m}, 1.0 \mathrm{~h}, 2.0 \mathrm{~h}$ \\
\hline 8 & 768.0h & $1.0 \mathrm{~m}, 15.0 \mathrm{~m}, 30.0 \mathrm{~m}, 1.0 \mathrm{~h}, 2.0 \mathrm{~h}$ \\
\hline 9 & 1536.0h & $1.0 \mathrm{~m}, 15.0 \mathrm{~m}, 30.0 \mathrm{~m}, 1.0 \mathrm{~h}, 2.0 \mathrm{~h}$ \\
\hline 10 & $720.0 \mathrm{~h}$ & $20.0 \mathrm{~m}, 1.0 \mathrm{~h}, 12.0 \mathrm{~h}, 36.0 \mathrm{~h}$ \\
\hline
\end{tabular}

\subsection{SPCR6918TEST}

This suite contains 44 individual tests designed to verify proper function of Percent Inventory models with the number multicomponent material mode and all the PIR release modes relative to the same scenarios using simple and groups material modes. Some aspects of these tests are covered in the PIRModelDefsTest suite, (Section 4.4), but this suite focuses on expected results in a typical NRC moderate accident scenario at Watts Bar-1 and an abbreviated version of that scenario. Moreover, this suite includes NFAC-RTH-SCIPUFF integration tests with plot output results as test artifacts. NFAC-RTH-SCIPUFF integration tests request areas of contours for the following fields:

\begin{tabular}{ccccc} 
Class & Choice & Kind & Category & Type \\
\hline $\begin{array}{c}\text { Isotope Ground Deposition } \\
\text { RTH Radiation Field }\end{array}$ & Total Activity & & Surface Data & Mean Value (M) \\
Total Effective Dose & Rate & Surface Data & Mean Value (M)
\end{tabular}

The four scenarios over which the nine combinations of material and PIR release modes are repeated are defined as follows.

\subsubsection{No Shutdown Full}

This is the Watts Bar-1 moderate accident scenario with the shutdown period removed.

\begin{tabular}{|c|c|c|c|c|c|c|c|c|c|}
\hline & ility: & & Watts & 3ar-2 (PV & & & & & \\
\hline & rating $\mathrm{Po}$ & ver: & 3480. & $\mathrm{MWt}$ & & & & & \\
\hline & erial Mod & & Grour & & & & & & \\
\hline & Release & Mode: & Avera & & & & & & \\
\hline & Irce Term & & Perce & t Inventor & & & & & \\
\hline & itdown Dı & ration: & 0 & & & & & & \\
\hline & $\begin{array}{l}\text { ease Dur } \\
\text { eases: }\end{array}$ & tion: & $44.9 \mathrm{r}$ & & & & & & \\
\hline \# & Duration & $N G$ & AM & $\mathrm{AE}$ & Hal & Chal & Plat & Tetraval & Trival \\
\hline 0 & $1.9 \mathrm{~h}$ & 3.0 & 0.003 & $1.0 e-5$ & 0.003 & 0.03 & $1.2 \mathrm{e}-7$ & 0 & 0 \\
\hline 1 & $32.0 \mathrm{~h}$ & 3.0 & 0.003 & 0 & 0.003 & 0.03 & $1.68 \mathrm{e}-6$ & $4.0 \mathrm{e}-7$ & $6.0 e-7$ \\
\hline 2 & $11.0 \mathrm{~h}$ & 17.0 & 0.07 & $8.95 e-5$ & 0.089 & 0.64 & $6.2 e-6$ & 7.0e-7 & $1.6 e-6$ \\
\hline
\end{tabular}




\subsubsection{No Shutdown Partial}

This is the Watts Bar-1 moderate accident scenario with the shutdown period removed, the second phased release removed, and the final phase reduced to a duration of $3.0 \mathrm{~h}$.

\begin{tabular}{|c|c|c|c|c|c|c|c|c|}
\hline Facility: & & Watts & Bar-2 (PV & & & & & \\
\hline Operating Pc & ver: & 3480. & $\mathrm{MWt}$ & & & & & \\
\hline Material Moc & & Group & & & & & & \\
\hline PIR Release & Mode: & Avera & & & & & & \\
\hline Source Term & & Perce & It Inventol & & & & & \\
\hline Shutdown D & ation: & 0 & & & & & & \\
\hline $\begin{array}{l}\text { Release Dur } \\
\text { Releases: }\end{array}$ & tion: & $44.9 \mathrm{r}$ & & & & & & \\
\hline \# Duration & $N G$ & AM & $\mathrm{AE}$ & Hal & Chal & Plat & Tetraval & Trival \\
\hline $1.9 \mathrm{~h}$ & 3.0 & 0.003 & $1.0 e-5$ & 0.003 & 0.03 & $1.2 \mathrm{e}-7$ & 0 & 0 \\
\hline $3.0 \mathrm{~h}$ & 17.0 & 0.07 & $8.95 e-5$ & 0.089 & 0.64 & $6.2 e-6$ & $7.0 \mathrm{e}-7$ & $1.6 e-6$ \\
\hline
\end{tabular}

\subsubsection{Shutdown Full}

This is the Watts Bar-1 moderate accident scenario as defined by the NRC.

\begin{tabular}{|c|c|c|c|c|c|c|c|c|c|}
\hline & ility: & & Watts & 3ar-2 (PV & & & & & \\
\hline & erating $\mathrm{Pc}$ & ver: & 3480. & $\mathrm{MWt}$ & & & & & \\
\hline & terial Moo & & Group & & & & & & \\
\hline & Release & Mode: & Avera & & & & & & \\
\hline & ırce Term & & Perce & t Invento & & & & & \\
\hline & itdown Dı & ration: & $3.1 \mathrm{~h}$ & & & & & & \\
\hline & $\begin{array}{l}\text { ease Dur } \\
\text { eases: }\end{array}$ & tion: & $44.9 \mathrm{r}$ & & & & & & \\
\hline \# & Duration & $N G$ & AM & $A E$ & $\mathrm{Hal}$ & Chal & Plat & Tetraval & Trival \\
\hline 0 & $1.9 \mathrm{~h}$ & 3.0 & 0.003 & $1.0 e-5$ & 0.003 & 0.03 & $1.2 \mathrm{e}-7$ & 0 & 0 \\
\hline 1 & $32.0 \mathrm{~h}$ & 3.0 & 0.003 & 0 & 0.003 & 0.03 & $1.68 e-6$ & $4.0 e-7$ & $6.0 e-7$ \\
\hline 2 & $11.0 \mathrm{~h}$ & 17.0 & 0.07 & $8.95 e-5$ & 0.089 & 0.64 & $6.2 e-6$ & $7.0 \mathrm{e}-7$ & $1.6 e-6$ \\
\hline
\end{tabular}

\subsubsection{Shutdown Partial}

This is the Watts Bar- 1 moderate accident scenario with the second phased release removed, and the final phase reduced to a duration of $3.0 \mathrm{~h}$. 


\begin{tabular}{|c|c|c|c|c|c|c|c|c|}
\hline Facility: & & Watts & Bar-2 (PV & & & & & \\
\hline Operating Pc & ver: & 3480. & $\mathrm{MWt}$ & & & & & \\
\hline Material Moc & & Group & & & & & & \\
\hline PIR Release & Mode: & Avera & & & & & & \\
\hline Source Term & & Perce & t Invento & & & & & \\
\hline Shutdown D & ation: & $3.1 \mathrm{~h}$ & & & & & & \\
\hline $\begin{array}{l}\text { Release Dur } \\
\text { Releases: }\end{array}$ & tion: & $44.9 \mathrm{r}$ & & & & & & \\
\hline \# Duration & NG & AM & $\mathrm{AE}$ & Hal & Chal & Plat & Tetraval & Trival \\
\hline $1.9 \mathrm{~h}$ & 3.0 & 0.003 & $1.0 e-5$ & 0.003 & 0.03 & $1.2 e-7$ & 0 & 0 \\
\hline $3.0 \mathrm{~h}$ & 17.0 & 0.07 & $8.95 e-5$ & 0.089 & 0.64 & $6.2 e-6$ & $7.0 \mathrm{e}-7$ & $1.6 e-6$ \\
\hline
\end{tabular}

\section{Test:testNoShutdownFullGroupsAverage}

An NFAC-RTH integration test, this exercises the "No Shutdown Full" scenario with groups material mode and average PIR release mode.

\section{Test:testNoShutdownFullGroupsExplicit}

An NFAC-RTH integration test, this exercises the "No Shutdown Full" scenario with groups material mode and explicit PIR release mode.

\section{Test:testNoShutdownFullGroupsNone}

An NFAC-RTH integration test, this exercises the "No Shutdown Full" scenario with groups material mode and none PIR release mode.

\section{Test:testNoShutdownFullMCAverage}

An NFAC-RTH integration test, this exercises the "No Shutdown Full" scenario with multicomponent material mode and average PIR release mode.

\section{Test:testNoShutdownFullMCExplicit}

An NFAC-RTH integration test, this exercises the "No Shutdown Full" scenario with multicomponent material mode and explicit PIR release mode.

\section{Test:testNoShutdownFullMCNone}

An NFAC-RTH integration test, this exercises the "No Shutdown Full" scenario with multicomponent material mode and none PIR release mode.

\section{Test:testNoShutdownFullMCNoneContours}

An NFAC-RTH-SCIPUFF integration test, this exercises the "No Shutdown Full" scenario with multicomponent material mode and none PIR release mode and compares resulting contour areas. 


\section{Test:testNoShutdownFullSimpleAverage}

An NFAC-RTH integration test, this exercises the "No Shutdown Full" scenario with simple material mode and average PIR release mode.

\section{Test:testNoShutdownFullSimpleExplicit}

An NFAC-RTH integration test, this exercises the "No Shutdown Full" scenario with simple material mode and explicit PIR release mode.

\section{Test:testNoShutdownFullSimpleNone}

An NFAC-RTH integration test, this exercises the "No Shutdown Full" scenario with simple material mode and none PIR release mode.

\section{Test:testNoShutdownPartialGroupsAverage}

An NFAC-RTH integration test, this exercises the "No Shutdown Partial" scenario with groups material mode and average PIR release mode.

\section{Test:testNoShutdownPartialGroupsExplicit}

An NFAC-RTH integration test, this exercises the "No Shutdown Partial" scenario with groups material mode and explicit PIR release mode.

\section{Test:testNoShutdownPartialGroupsNone}

An NFAC-RTH integration test, this exercises the "No Shutdown Partial" scenario with groups material mode and none PIR release mode.

\section{Test:testNoShutdownPartialGroupsNoneContours}

An NFAC-RTH-SCIPUFF integration test, this exercises the "No Shutdown Partial" scenario with groups material mode and none PIR release mode and compares resulting contour areas.

\section{Test:testNoShutdownPartialMCAverage}

An NFAC-RTH integration test, this exercises the "No Shutdown Partial" scenario with multicomponent material mode and average PIR release mode.

\section{Test:testNoShutdownPartialMCExplicit}

An NFAC-RTH integration test, this exercises the "No Shutdown Partial" scenario with multicomponent material mode and explicit PIR release mode.

\section{Test:testNoShutdownPartialMCNone}

An NFAC-RTH integration test, this exercises the "No Shutdown Partial" scenario with multicomponent material mode and none PIR release mode. 


\section{Test:testNoShutdownPartialMCNoneContours}

An NFAC-RTH-SCIPUFF integration test, this exercises the "No Shutdown Partial" scenario with multicomponent material mode and none PIR release mode and compares resulting contour areas.

\section{Test:testNoShutdownPartialSimpleAverage}

An NFAC-RTH integration test, this exercises the "No Shutdown Partial" scenario with simple material mode and average PIR release mode.

\section{Test:testNoShutdownPartialSimpleExplicit}

An NFAC-RTH integration test, this exercises the "No Shutdown Partial" scenario with simple material mode and explicit PIR release mode.

\section{Test:testNoShutdownPartialSimpleNone}

An NFAC-RTH integration test, this exercises the "No Shutdown Partial" scenario with simple material mode and none PIR release mode.

\section{Test:testNoShutdownPartialSimpleNoneContours}

An NFAC-RTH-SCIPUFF integration test, this exercises the "No Shutdown Partial" scenario with simple material mode and none PIR release mode and compares resulting contour areas.

\section{Test:testShutdownFullGroupsAverage}

An NFAC-RTH integration test, this exercises the "Shutdown Full" scenario with groups material mode and average PIR release mode.

\section{Test:testShutdownFullGroupsExplicit}

An NFAC-RTH integration test, this exercises the "Shutdown Full" scenario with groups material mode and explicit PIR release mode.

\section{Test:testShutdownFullGroupsNone}

An NFAC-RTH integration test, this exercises the "Shutdown Full" scenario with groups material mode and none PIR release mode.

\section{Test:testShutdownFullMCAverage}

An NFAC-RTH integration test, this exercises the "Shutdown Full" scenario with multicomponent material mode and average PIR release mode.

\section{Test:testShutdownFullMCExplicit}

An NFAC-RTH integration test, this exercises the "Shutdown Full" scenario with multicomponent material mode and explicit PIR release mode. 


\section{Test:testShutdownFullMCNone}

An NFAC-RTH integration test, this exercises the "Shutdown Full" scenario with multicomponent material mode and none PIR release mode.

\section{Test:testShutdownFullMCNoneContours}

An NFAC-RTH-SCIPUFF integration test, this exercises the "Shutdown Full" scenario with multicomponent material mode and none PIR release mode and compares resulting contour areas.

\section{Test:testShutdownFullSimpleAverage}

An NFAC-RTH integration test, this exercises the "Shutdown Full" scenario with simple material mode and average PIR release mode.

\section{Test:testShutdownFullSimpleExplicit}

An NFAC-RTH integration test, this exercises the "Shutdown Full" scenario with simple material mode and explicit PIR release mode.

\section{Test:testShutdownFullSimpleNone}

An NFAC-RTH integration test, this exercises the "Shutdown Full" scenario with simple material mode and none PIR release mode.

\section{Test:testShutdownPartialGroupsAverage}

An NFAC-RTH integration test, this exercises the "Shutdown Partial" scenario with groups material mode and average PIR release mode.

\section{Test:testShutdownPartialGroupsExplicit}

An NFAC-RTH integration test, this exercises the "Shutdown Partial" scenario with groups material mode and explicit PIR release mode.

\section{Test:testShutdownPartialGroupsNone}

An NFAC-RTH integration test, this exercises the "Shutdown Partial" scenario with groups material mode and none PIR release mode.

\section{Test:testShutdownPartialGroupsNoneContours}

An NFAC-RTH-SCIPUFF integration test, this exercises the "Shutdown Partial" scenario with groups material mode and none PIR release mode and compares resulting contour areas.

\section{Test:testShutdownPartialMCAverage}

An NFAC-RTH integration test, this exercises the "Shutdown Partial" scenario with multicomponent material mode and average PIR release mode. 


\section{Test:testShutdownPartialMCExplicit}

An NFAC-RTH integration test, this exercises the "Shutdown Partial" scenario with multicomponent material mode and explicit PIR release mode.

\section{Test:testShutdownPartialMCNone}

An NFAC-RTH integration test, this exercises the "Shutdown Partial" scenario with multicomponent material mode and none PIR release mode.

\section{Test:testShutdownPartialMCNoneContours}

An NFAC-RTH-SCIPUFF integration test, this exercises the "Shutdown Partial" scenario with multicomponent material mode and none PIR release mode and compares resulting contour areas.

\section{Test:testShutdownPartialSimpleAverage}

An NFAC-RTH integration test, this exercises the "Shutdown Partial" scenario with simple material mode and average PIR release mode.

\section{Test:testShutdownPartialSimpleExplicit}

An NFAC-RTH integration test, this exercises the "Shutdown Partial" scenario with simple material mode and explicit PIR release mode.

\section{Test:testShutdownPartialSimpleNone}

An NFAC-RTH integration test, this exercises the "Shutdown Partial" scenario with simple material mode and none PIR release mode.

\section{Test:testShutdownPartialSimpleNoneContours}

An NFAC-RTH-SCIPUFF integration test, this exercises the "Shutdown Partial" scenario with simple material mode and none PIR release mode and compares resulting contour areas.

\section{INTERACTIVE TESTS}

The purpose of the interactive tests is to exercise each piece of functionality in the Nuclear Facility Incident Edit dialog and its constituent components. Verification of results is by visual inspection of the interface and/or examination of project files after being saved and are recorded in the test descriptions below.

\subsection{TOP LEVEL INTERACTIONS}

\section{Test: Set Incident Name}

Set the incident name via the Name edit field.

Verification:

- Incident name correctly (de)serialized from/to the project file. 


\subsection{WHERE TAB}

\section{Test: Select Facility}

Select a facility via the Select button in the Facility group. Examine the facility tree to verify correct representation of the facilities defined in NFAC data files.

Verification:

- The All Facilities tree lists all the countries (and generic) represented in the wcountry.dat file.

- Each country tree node expands to the proper subtrees (Power Reactor, Reprocessing Facility, and/or Research Reactor) based on facilities available in that country.

- Verify special statuses decommissioned, not yet completed, and no inventory are accurately represented.

- Each leaf node lists the available facilities correctly as per the associated lwr_??.dat file.

- The selected facility is correctly represented and (de)serialized from/to the project file.

\section{Test: Select Default Facility Location}

Choose the Facility Location radio button in the Location group for the default facility location. Verification:

- The coordinate for the facility as stored in the facility database is (de)serialized from/to the project file.

\section{Test: Enter Explicit Facility Location}

Selected the Customized Location radio button and enter an explicit coordinate for the facility location.

Verification:

- The entered coordinate is (de)serialized from/to the project file as the facility location.

\section{Test: Define Facility Location by Dragging the Incident Icon}

With the Customized Location radio button selected, drag the incident icon on the map to set the location.

Verification:

- The dragged-to coordinate is (de)serialized from/to the project file as the facility location.

\section{Test: Select the Default Inventory File}

Choose the Default for Facility radio button in the Inventory group. Verification:

- The inventory file referenced in the facility database entry is (de)serialized from/to the project file. 


\section{Test: Select a Custom Inventory File}

Choose the Customized radio button in the Inventory group and specify an inventory (i.e., activity vector or .avc) file.

Verification:

- The specified file is (de)serialized from/to the project file as the custom inventory.

\section{Test: Use the ORIGEN File Import Utility to Create a Custom Inventory File}

Activate the Origen Import button to run the ORIGEN Importer. Verification:

- After selecting a F71 file, the ORIGEN File Import utility appears and displays the cases in the file.

- Activating the Show File Overview button brings up an overview windowing showing information about each case in the F71 file.

- After selecting a case and activating the Show Case Overview button, a window giving a summary of the selected case appears.

- After selecting a case and activating the Show Case Details button, a windowing displaying details of the selected case appears.

- After selecting a case, selecting and entering values in the Inventory File Units group, and activating the Create Inventory File button, and entering a target filename, an inventory file is created.

- The created inventory file can be specified as the custom inventory for the facility.

\subsection{WHAT TAB}

\section{Test: Enter an Explicit Operating Power}

Change the value of the Operating Power field.

Verification:

- The specified operating power is (de)serialized from/to the project file.

\section{Test: Select a Moderate Incident}

Choose the Moderate Incident radio button.

Verification:

- The Moderate model appropriate for the selected facility is (de)serialized from/to the project file and displayed in the report resulting from activating the View Source Term button (compared to the associated acmxxx.dat file).

\section{Test: Select a Severe Incident}

Choose the Severe Incident radio button.

Verification:

- The Severe model appropriate for the selected facility is (de)serialized from/to the project file and displayed in the report resulting from activating the View Source Term button (compared to the associated acsxxx.dat file). 


\section{Test: Define an Isotopic Release Rates Incident}

Choose the Technical Analysis radio button. Activate the Define Incident button and choose Isotopic Release Rates from the Source Terms Available list. Click the Next button. From the Define Isotopic Release Rates dialog, choose Release Units and enter values under Isotope Values.

Verification:

- The defined isotope values and release units are (de)serialized from/to the project file and displayed in the report resulting from activating the View Source Term button.

\section{Test: Define an Isotopic Concentrations Incident}

Choose the Technical Analysis radio button. Activate the Define Incident button and choose Isotopic Concentrations from the Source Terms Available list. Click the Next button. From the Define Isotopic Release Rates dialog, choose Release Units and enter values under Isotope Values.

Verification:

- The defined isotope values and release units are (de)serialized from/to the project file and displayed in the report resulting from activating the View Source Term button.

\section{Test: Define a Percent Inventory Incident}

Choose the Technical Analysis radio button. Activate the Define Incident button and choose Percent Inventory from the Source Terms Available list. Click the Next button. From the Define Percent Inventory Releases dialog, activate the Load button and choose From Moderate Model. Then, activate the Load button and choose From Severe Model. Enter explicit values in the release duration and group percentage cells. Activate the New button to add a release. Change the duration units via the Duration Units combo box. Select a cell in any release column and activate Delete to remove that release.

Verification:

- The release definitions for the moderate and severe models are correct for the type of facility acmxxx.dat and acs xxx.dat files, respectively).

- The (first) totals column is accurately updated after cell edits.

- All duration cell values are converted upon selection of new duration units.

- A new, release column is added when New is activated with cell values set to the remainder required to reach $100 \%$ release of each group.

- Deleted releases are moved.

- The defined releases are (de)serialized from/to the project file and displayed in the report resulting from activating the View Source Term button.

\section{Test: Define an Analyst Mix Incident}

Choose the Technical Analysis radio button. Activate the Define Incident button and choose Mix Specified by Analyst from the Source Terms Available list. Click the Next button. From the Define Mix Specified by Analyst dialog, enter a Gross Release Rate and various values for the percentages by MELCOR group.

Verification: 
- The Gross Release Rate value is (de)serialized from/to the project file.

- Entering release percentages totaling more than $100 \%$ results in a warning dialog.

- Release percentages for each group are (de)serialized from/to the project file.

\section{Test: Define a Containment Monitor Reading Incident}

Choose the Technical Analysis radio button. Activate the Define Incident button and choose Mix Specified by Analyst from the Source Terms Available list. Click the Next button. From the Define Containment Monitor Reading Incident dialog, enter various values for: Representative Operating Power, Monitor Reading and Location, Sprays, Release Path, and Leak Rate.

Verification:

- All entered values are (de)serialized from/to the project file.

- A warning appears if $100 \%$ leak rate is selected with a filtered release path.

- The release duration is correctly calculated from the specified leak rate.

\section{Test: Define a Spent Fuel Incident}

Choose the Technical Analysis radio button. Activate the Define Incident button and choose Spent Fuel from the Source Terms Available list. Click the Next button. From the Define Spent Fuel/Spent Fuel Pool Incident dialog, enter various values for: Representative Operating Power, Number of Batches, Fuel Condition, Sprays, Release Path, Last Batch in Pool datetime, and Leak Rate.

Verification:

- All entered values are (de)serialized from/to the project file.

- A warning appears if $100 \%$ leak rate is selected with a filtered release path.

- The Last Batch in Pool time is forced to be no later than Shutdown/Start of Decay on the When tab.

- The release duration is correctly calculated from the specified leak rate.

\section{Test: Define a RASCAL Project Incident}

Choose the Technical Analysis radio button. Activate the Define Incident button and choose RASCAL Project from the Source Terms Available list. Click the Next button. From the RASCAL Project dialog, activate the Read File button to locate and select a RASCAL project file.

Verification:

- The contained tadspecs.tmp file is read correctly to get the case name.

- The contained NucName.tmp file is read correctly to get the list of isotopes.

- The contained STC_Rel.tmp file is read correctly to get release durations and activity values for each isotope at each release time.

- The isotope activities and durations for each release are correctly (de)serialized from/to the project file. 


\section{Test: Define a Confinement Bypass Plant Conditions Incident}

In the Where tab, choose a facility for which the Confinement Bypass plant condition is available, such as Hartlepool-1 in Great Britain (an AGR). On the What tab, choose the Technical Analysis radio button. Activate the Define Incident button, choose Plant Conditions from the Source Terms Available list, activate the Next button, and then choose Bypass of Confinement from the Plant Conditions Available list. From the Bypass of Confinement dialog, enter or select various values for Representative Operating Power, Core Condition, and Leak Rate.

Verification:

- Values entered or selected are (de)serialized from/to the project file.

- The release duration is correctly calculated from the specified leak rate.

\section{Test: Define a Confinement Leakage/Failure Plant Conditions Incident}

In the Where tab, choose a facility for which the Confinement Bypass plant condition is available, such as Novovoronezh-3 in Russia (a VVER-440/230). On the What tab, choose the Technical Analysis radio button. Activate the Define Incident button, choose Plant Conditions from the Source Terms Available list, activate the Next button, and then choose Confinement Leakage/Failure from the Plant Conditions Available list. From the Confinement Leakage/Failure dialog, enter or select various values for Representative Operating Power, Core Condition, and Leak Rate.

Verification:

- Values entered or selected are (de)serialized from/to the project file.

- The release duration is correctly calculated from the specified leak rate.

\section{Test: Define a Containment Bypass Plant Conditions Incident}

In the Where tab, choose a facility for which the Containment Bypass plant condition is available, such as Hamaoka-3 in Japan (a BWR Mk-1). On the What tab, choose the Technical Analysis radio button. Activate the Define Incident button, choose Plant Conditions from the Source Terms Available list, activate the Next button, and then choose Bypass of Containment from the Plant Conditions Available list. From the Bypass of Containment dialog, enter or select various values for Representative Operating Power, Core Condition, Release Path, and Leak Rate.

Verification:

- Values entered or selected are (de)serialized from/to the project file.

- The release duration is correctly calculated from the specified leak rate.

\section{Test: Define a Containment Leakage/Failure Plant Conditions Incident}

In the Where tab, choose a facility for which the Containment Leakage plant condition is available, such as Beloyarsky-3(BN-600) in Russia (an LMFBR, requires a custom inventory file). On the What tab, choose the Technical Analysis radio button. Activate the Define Incident button, choose Plant Conditions from the Source Terms Available list, activate the Next button, and then choose Containment Leakage/Failure from the Plant Conditions Available list. From the Containment Leakage/Failure dialog, enter or select various values for Representative Operating Power, Core Condition, Release Path, and Leak Rate.

Verification: 
- Values entered or selected are (de)serialized from/to the project file.

- The release duration is correctly calculated from the specified leak rate.

\section{Test: Define a Dry Well Leakage/Failure Plant Conditions Incident}

In the Where tab, choose a facility for which the Dry Well Leakage plant condition is available, such as Hamaoka-3 in Japan (a BWR Mk-1). On the What tab, choose the Technical Analysis radio button. Activate the Define Incident button, choose Plant Conditions from the Source Terms Available list, activate the Next button, and then choose Dry Well Leakage/Failure from the Plant Conditions Available list. From the Dry Well Leakage/Failure dialog, enter or select various values for Representative Operating Power, Core Condition, Release Path, Sprays, and Leak Rate.

Verification:

- Values entered or selected are (de)serialized from/to the project file.

- The release duration is correctly calculated from the specified leak rate.

\section{Test: Define an Ice Condenser Containment Leakage/Failure Plant Conditions Incident}

In the Where tab, choose a facility for which the Ice Condenser Containment plant condition is available, such as Watts Bar-1 in the United States (a PWR). On the What tab, choose the Technical Analysis radio button. Activate the Define Incident button, choose Plant Conditions from the Source Terms Available list, activate the Next button, and then choose Ice Condenser Containment Leakage/Failure from the Plant Conditions Available list. From the Ice Condenser Containment Leakage/Failure dialog, enter or select various values for Representative Operating Power, Core Condition, Release Path, Sprays, Fans, Ice Bed Condition Before Core Damage, and Leak Rate.

Verification:

- Values entered or selected are (de)serialized from/to the project file.

- The release duration is correctly calculated from the specified leak rate.

\section{Test: Define a Large Dry Subcontainment Leakage/Failure Plant Conditions Incident}

In the Where tab, choose a facility for which the Large Dry Subcontainment Leakage plant condition is available, such as Beaver Valley-1 in the United States (a PWR). On the What tab, choose the Technical Analysis radio button. Activate the Define Incident button, choose Plant Conditions from the Source Terms Available list, activate the Next button, and then choose Large, Dry, or Subatmospheric Containment Leakage/Failure from the Plant Conditions Available list. From the Large, Dry, or Subatmospheric Containment Leakage/Failure dialog, enter or select various values for Representative Operating Power, Core Condition, Release Path, Sprays, and Leak Rate.

Verification:

- Values entered or selected are (de)serialized from/to the project file.

- The release duration is correctly calculated from the specified leak rate. 


\section{Test: Define a Prompt Critical Power Excursion Plant Conditions Incident}

In the Where tab, choose a facility for which the Prompt Critical Power Excursion plant condition is available, such as Bilibino Unit A in Russia (an RBMK). On the What tab, choose the Technical Analysis radio button. Activate the Define Incident button, choose Plant Conditions from the Source Terms Available list, activate the Next button, and then choose Prompt Critical Power Excursion from the Plant Conditions Available list. From the Prompt Critical Power Excursion dialog, enter or select various values for Representative Operating Power, and Core Involvement.

Verification:

- Values entered or selected are (de)serialized from/to the project file.

\section{Test: Define a Reprocessing Facility Plant Conditions Incident}

In the Where tab, choose a facility for which the Reprocessing Facility plant condition is available, such as Savannah River in the United States. On the What tab, choose the Technical Analysis radio button. Activate the Define Incident button, choose Plant Conditions from the Source Terms Available list, activate the Next button, and then choose Reprocessing Facility Plant Conditions from the Plant Conditions Available list. From the Reprocessing Facility Plant Conditions dialog, enter or select various values for Plant Throughput, Release Rate, and the values in the Release Fraction by Component group.

Representative Operating Power, and Core Involvement.

Verification:

- Values entered or selected are (de)serialized from/to the project file.

\section{Test: Define a Steam Generator Tube Rupture Plant Conditions Incident}

In the Where tab, choose a facility for which the Steam Tube Rupture plant condition is available, such as Dungeness B-1 in Great Britain (an AGR). On the What tab, choose the Technical Analysis radio button. Activate the Define Incident button, choose Plant Conditions from the Source Terms Available list, activate the Next button, and then choose Steam Generator Tube Rupture (Coolant) from the Plant Conditions Available list. From the Steam Generator Rube Rupture (Coolant) dialog, enter or select various values for Coolant Concentration, Steam Generator Conditions, Release is From, and Release Rate.

Verification:

- Values entered or selected are (de)serialized from/to the project file.

\section{Test: Define a Subatmospheric Confinement Leakage/Failure Plant Conditions Incident}

In the Where tab, choose a facility for which the Subatmospheric Confinement Leakage/Failure plant condition is available, such as Rovno-1 in the Ukraine (VVER-440/213). On the What tab, choose the Technical Analysis radio button. Activate the Define Incident button, choose Plant Conditions from the Source Terms Available list, activate the Next button, and then choose Subatmospheric Confinement Leakage/Failure from the Plant Conditions Available list. From the Subatmospheric Confinement Leakage/Failure dialog, enter or select various values for Representative Operating Power, Core Condition, Pool Suppression System, and Leak Rate.

Verification: 
- Values entered or selected are (de)serialized from/to the project file.

- The release duration is correctly calculated from the specified leak rate.

\section{Test: Define a Wet Well Leakage/Failure Plant Conditions Incident}

In the Where tab, choose a facility for which the Wet Well Leakage plant condition is available, such as Hamaoka-3 in Japan (a BWR Mk-1). On the What tab, choose the Technical Analysis radio button. Activate the Define Incident button, choose Plant Conditions from the Source Terms Available list, activate the Next button, and then choose Wet Well Leakage/Failure from the Plant Conditions Available list. From the Wet Well Leakage/Failure dialog, enter or select various values for Representative Operating Power, Core Condition, Release Path, Wet Well, and Leak Rate.

Verification:

- Values entered or selected are (de)serialized from/to the project file.

- The release duration is correctly calculated from the specified leak rate.

\section{Test: Select Material Processing Modes}

Step 1. From the Where tab, select the facility Bruce-1 (Canada $\rightarrow$ Power Reactor). On the What tab activate the Edit Calculation Options button to bring up the Edit Calculation Options dialog. Check that the Mode combo box in the Material Processing group is disabled. Verification:

- The combo box is disabled.

Step 2. From the Where tab, select the facility Beaver Valley-1 Bruce-1 (United States $\rightarrow$ Power Reactor). On the What tab activate the Technical Analysis radio button. Activate the Edit Calculation Options button to bring up the Edit Calculation Options dialog. In turn for each option, do the following.

Select the mode. Activate the OK button to close dialog. Select File $\rightarrow$ Export Project to ASCII from the menu bar. After saving the file, examine it with an editor to verify the materialMode field has the correct value.

Verification:

- The combo box is enabled.

- Three modes are available: Simple, Groups, Multicomponent.

- The materialMode field value is correct in the ASCII project export.

\section{Test: Select Percent Inventory Release Modes}

On the What tab activate the Technical Analysis radio button. Activate the Edit Calculation Options button to bring up the Edit Calculation Options dialog. Activate the Mode combo box in the Percent Inventory Release group. In turn for each option, do the following.

Select the mode. Activate the OK button to close dialog. Select File $\rightarrow$ Export Project to ASCII from the menu bar. After saving the file, examine it with an editor to verify the PIRMode field has the correct value.

Verification:

- Three modes are available: Average, Explicit, None.

- The PIRMode field value is correct in the ASCII project export. 


\section{Test: View Source Term}

Activate the View Source Term button to generate and display an HTML report giving details of the source term as currently defined.

Verification:

- Source term HTML report generated for all source models.

\subsection{WHEN TAB}

The When tab in the NFAC GUI is relatively complex compared to other incident source models. Refer to Section 3.4 ModelTimesTest for a discription of the six event times possible in NFAC incidents. Which of the event times are visible and/or editable for a particular NFAC source model depends on that model and which of the event times are defined or computed based on the model definition. Further, the new Advanced Mode toggle button allows the user to override these settings and enter explicit times for all events, possibly redefining the model in the process. The interactive tests examine each NFAC source model to verify the proper times are visible and editable and are correctly computed if appropriate. The Reset Times button is activated to ensure the default times are applied.

\section{Test: Moderate Model Times Edit}

Verification:

$\begin{array}{ll}\text { Shutdown/Start of Decay: } & \text { visible, editable } \\ \text { Release to Containment: } & \text { invisible } \\ \text { Release to Environment: } & \text { visible, uneditable, calculated correctly } \\ \text { End of Release: } & \text { visible, uneditable, calculated correctly } \\ \text { End of Dispersion: } & \text { visible, editable } \\ \text { End of Exposure: } & \text { visible, editable } \\ \text { After reset, times set correctly }\end{array}$

\section{Test: Severe Model Times Edit}

Verification:

$\begin{array}{ll}\text { Shutdown/Start of Decay: } & \text { visible, editable } \\ \text { Release to Containment: } & \text { invisible } \\ \text { Release to Environment: } & \text { visible, uneditable, calculated correctly } \\ \text { End of Release: } & \text { visible, uneditable, calculated correctly } \\ \text { End of Dispersion: } & \text { visible, editable } \\ \text { End of Exposure: } & \text { visible, editable } \\ \text { After reset, times set correctly }\end{array}$

\section{Test: Analyst Mix Model Times Edit}

Verification: 


\begin{abstract}
Shutdown/Start of Decay: visible, editable
Release to Containment: invisible

Release to Environment: visible, editable

End of Release: visible, editable

End of Dispersion: visible, editable

End of Exposure: visible, editable

After reset, times set correctly
\end{abstract}

\title{
Test: Containment Monitor Reading Model Times Edit
}

Verification:

Shutdown/Start of Decay: invisible

Release to Containment: visible, editable

Release to Environment: visible, editable

End of Release: visible, editable ${ }^{1}$

End of Dispersion: visible, editable

End of Exposure: visible, editable

After reset, times set correctly

1 Time can be manually set less than computed end time based on leak rate

\section{Test: Isotopic Concentrations Model Times Edit}

Verification:

Shutdown/Start of Decay: invisible

Release to Containment: invisible

Release to Environment: visible, editable

End of Release: visible, editable

End of Dispersion: visible, editable

End of Exposure: visible, editable

After reset, times set correctly

\section{Test: Isotopic Release Rates Model Times Edit}

Verification:

$\begin{array}{ll}\text { Shutdown/Start of Decay: } & \text { invisible } \\ \text { Release to Containment: } & \text { invisible } \\ \text { Release to Environment: } & \text { visible, editable } \\ \text { End of Release: } & \text { visible, editable } \\ \text { End of Dispersion: } & \text { visible, editable } \\ \text { End of Exposure: } & \text { visible, editable }\end{array}$

After reset, times set correctly

Test: Percent Inventory Model Times Edit

Verification: 


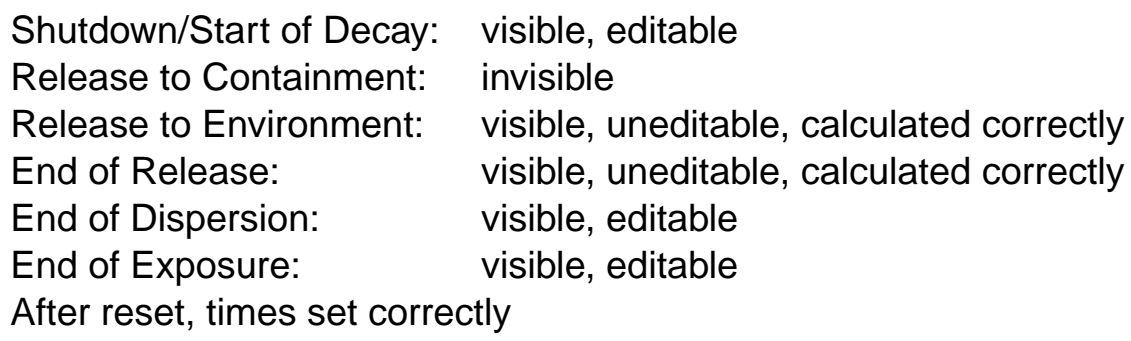

Test: Containment Bypass Plant Conditions Model Times Edit

Verification:

$\begin{array}{ll}\text { Shutdown/Start of Decay: } & \text { visible, editable } \\ \text { Release to Containment: } & \text { invisible } \\ \text { Release to Environment: } & \text { visible, editable } \\ \text { End of Release: } & \text { visible, editable }{ }^{1} \\ \text { End of Dispersion: } & \text { visible, editable } \\ \text { End of Exposure: } & \text { visible, editable }\end{array}$

After reset, times set correctly

1 Time can be manually set less than computed end time based on leak rate

\section{Test: Prompt Critical Power Excursion Plant Conditions Model Times Edit}

Verification:

$\begin{array}{ll}\text { Shutdown/Start of Decay: } & \text { invisible } \\ \text { Release to Containment: } & \text { invisible } \\ \text { Release to Environment: } & \text { visible, editable } \\ \text { End of Release: } & \text { visible, editable } \\ \text { End of Dispersion: } & \text { visible, editable } \\ \text { End of Exposure: } & \text { visible, editable }\end{array}$

After reset, times set correctly

Test: Reprocessing Facility Plant Conditions Model Times Edit

Verification:

$\begin{array}{ll}\text { Shutdown/Start of Decay: } & \text { invisible } \\ \text { Release to Containment: } & \text { invisible } \\ \text { Release to Environment: } & \text { visible, editable } \\ \text { End of Release: } & \text { visible, editable } \\ \text { End of Dispersion: } & \text { visible, editable } \\ \text { End of Exposure: } & \text { visible, editable }\end{array}$

After reset, times set correctly

\section{Test: Confinement Bypass Plant Conditions Model Times Edit}

Times management for this source model is shared by all other Plant Conditions models except for the three identified in the preceeding tests. 
Verification:

$\begin{array}{ll}\text { Shutdown/Start of Decay: } & \text { visible, editable } \\ \text { Release to Containment: } & \text { invisible } \\ \text { Release to Environment: } & \text { visible, editable } \\ \text { End of Release: } & \text { visible, editable }{ }^{1} \\ \text { End of Dispersion: } & \text { visible, editable } \\ \text { End of Exposure: } & \text { visible, editable }\end{array}$

After reset, times set correctly

1 Time can be manually set less than computed end time based on leak rate

Test: Spent Fuel Model Times Edit

Verification:

Shutdown/Start of Decay: invisible

Release to Containment: visible, editable

Release to Environment: visible, editable

End of Release: $\quad$ visible, editable ${ }^{1}$

End of Dispersion: visible, editable

End of Exposure: visible, editable

After reset, times set correctly

1 Time can be manually set less than computed end time based on leak rate

\section{Test: RASCAL Project Model Times Edit}

Verification:

$\begin{array}{ll}\text { Shutdown/Start of Decay: } & \text { invisible } \\ \text { Release to Containment: } & \text { invisible } \\ \text { Release to Environment: } & \text { visible, editable } \\ \text { End of Release: } & \text { visible, uneditable, calculated correctly } \\ \text { End of Dispersion: } & \text { visible, editable } \\ \begin{array}{ll}\text { End of Exposure: } & \text { visible, editable } \\ \text { After reset, times set correctly }\end{array}\end{array}$

\section{Test: Detect Event Times Out of Chronological Order}

After modification of editable time fields and activation of the OK button for the Nuclear Facility Incident Edit dialog, if times are not in chronological order, a "Times are not in chronological order" error dialog is displayed, and the model definition remains unchanged.

Verification:

- Out of order times are detected, a warning dialog is displayed, and the model definition is not updated. 


\section{Test: Apply Time Field Changes for All Events}

Enter a field value and activate the corresponding button in the Change Field for All Events group.

Verification:

- Month (MM) fields are updated.

- Day $(D D)$ fields are updated.

- Year fields are updated.

- Hours $(h h)$ fields are updated.

- Minutes $(\mathrm{mm})$ fields are updated.

- Seconds (ss) fields are updated.

- Calculated fields are updated correctly.

\section{Test: Override Times in Advanced Mode}

Activate the Advanced Mode toggle button to override editability of fields.

Verification:

- All visible fields are editable.

- Chronological order checks are still applied.

\subsection{NOTES TAB}

\section{Test: Edit Notes}

Verification:

- Notes are (de)serialized from/to the project file.

- Notes can be modified (changed, deleted, augmented). 


\section{RESULTS}

\subsection{AUTOMATED TEST RESULTS}

\section{NfacJUnit4Adapter consolidates all test results into a single report. The report is listed below.}

\begin{tabular}{|c|c|c|c|c|}
\hline \multicolumn{5}{|c|}{ Test project $\mathrm{z}: /$ re $7 \mathrm{x} / \mathrm{src} / \mathrm{hpac} 5$-poplib-cmake/build-win } \\
\hline $1 / 62$ & $\begin{array}{l}\text { Test \#1: } \\
\text { Start } 2:\end{array}$ & $\begin{array}{l}\text { test } 0001 \text { dc_around_tiles } \ldots \ldots \cdots \cdots \cdots \cdots \\
\text { test0002_dc_around_rasters }\end{array}$ & Passed & 0.64 \\
\hline $2 / 62$ & $\begin{array}{l}\text { Test \#2: } \\
\text { Start } 3:\end{array}$ & $\begin{array}{l}\text { test } 0002 \text { _dc_around_rasters } \ldots \ldots \ldots \ldots \ldots \ldots \cdots \\
\text { test0003_dc_inner_tiles }\end{array}$ & Passed & 0.75 \\
\hline $3 / 62$ & $\begin{array}{l}\text { Test \#3: } \\
\text { Start } 4:\end{array}$ & 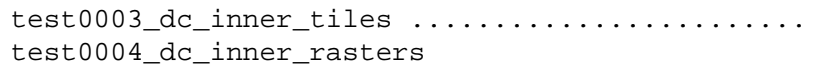 & Passed & 0.56 \\
\hline $4 / 62$ & $\begin{array}{l}\text { Test \# } 4: \\
\text { Start } 5:\end{array}$ & $\begin{array}{l}\text { test } 0004 \text { dc_inner_rasters } \ldots \ldots \cdots \cdots \cdots \cdots \cdots \\
\text { test0005_dc_diamond_tiles }\end{array}$ & Passed & 0.53 \\
\hline $5 / 62$ & $\begin{array}{l}\text { Test \#5: } \\
\text { Start } 6:\end{array}$ & $\begin{array}{l}\text { test } 0005 \text { ddc_diamond_tiles } \ldots \ldots \cdots \cdots \cdots \cdots \\
\text { test0006_dc_diamond_rasters }\end{array}$ & Passed & 0.56 \\
\hline $6 / 62$ & $\begin{array}{l}\text { Test \#6: } \\
\text { Start } 7:\end{array}$ & $\begin{array}{l}\text { test0006_dc_diamond_rasters } \ldots \ldots \ldots \ldots \cdots \\
\text { test0007_ny_triangle_tiles }\end{array}$ & Passed & 0.52 \\
\hline $7 / 62$ & $\begin{array}{l}\text { Test \#7: } \\
\text { Start } 8:\end{array}$ & $\begin{array}{l}\text { test } 0007 \text { ny_triangle_tiles } \ldots \cdots \cdots \cdots \cdots \cdots \\
\text { test0008_ny_triangle_rasters }\end{array}$ & Passed & 0.58 \\
\hline $8 / 62$ & $\begin{array}{l}\text { Test \#8: } \\
\text { Start 9: }\end{array}$ & $\begin{array}{l}\text { test } 0008 \text { _ny_triangle_rasters } \ldots \ldots \ldots \ldots \ldots \ldots \\
\text { test0009_va_exclude_1_tiles }\end{array}$ & Passed & 0.58 \\
\hline $9 / 62$ & $\begin{array}{l}\text { Test \#9: } \\
\text { Start 10: }\end{array}$ & $\begin{array}{l}\text { test 0009_va_exclude_1_tiles } \ldots \ldots \cdots \cdots \cdots \cdots \\
\text { test0010_va_exclude_1_tiles_ini }\end{array}$ & Passed & 1.55 \\
\hline $0 / 62$ & $\begin{array}{l}\text { Test \#10: } \\
\text { Start 11: }\end{array}$ & $\begin{array}{l}\text { test } 0010 \text { _va_exclude_1_tiles_ini } \ldots \ldots \ldots \ldots \ldots \\
\text { test0011_va_exclude_1_rasters }\end{array}$ & Passed & 1.52 \\
\hline $1 / 62$ & $\begin{array}{l}\text { Test \#11: } \\
\text { Start } 12:\end{array}$ & $\begin{array}{l}\text { test0011_va_exclude_1_rasters } \ldots \ldots \ldots \ldots \ldots \ldots \\
\text { test0012_va_exclude_1_rasters_ini }\end{array}$ & Passed & 1.80 \\
\hline $12 / 62$ & $\begin{array}{l}\text { Test \#12: } \\
\text { Start 13: }\end{array}$ & $\begin{array}{l}\text { test0012_va_exclude_1_rasters_ini } \ldots \ldots \ldots \ldots \\
\text { test0013_va_exclude_2_tiles }\end{array}$ & Passed & 1.83 \\
\hline $13 / 62$ & $\begin{array}{l}\text { Test \#13: } \\
\text { Start 14: }\end{array}$ & $\begin{array}{l}\text { test 0013_va_exclude_2_tiles } \ldots \ldots \cdots \cdots \cdots \cdots \cdots \\
\text { test0014_va_exclude_2_tiles_ini }\end{array}$ & Passed & 1.55 \\
\hline $14 / 62$ & $\begin{array}{l}\text { Test \#14: } \\
\text { Start 15: }\end{array}$ & $\begin{array}{l}\text { test 0014_va_exclude_2_tiles_ini } \ldots \ldots \ldots \cdots \cdots \\
\text { test0015_va_exclude_2_rasters }\end{array}$ & Passed & 1.62 \\
\hline $15 / 62$ & $\begin{array}{l}\text { Test \#15: } \\
\text { Start 16: }\end{array}$ & $\begin{array}{l}\text { test0015_va_exclude_2_rasters } \ldots \ldots \ldots \ldots \ldots \\
\text { test0016_va_exclude_2_rasters_ini }\end{array}$ & Passed & 1.59 \\
\hline $6 / 62$ & $\begin{array}{l}\text { Test \#16: } \\
\text { Start 17: }\end{array}$ & $\begin{array}{l}\text { test 0016_va_exclude_2_rasters_ini } \ldots \ldots \cdots \cdots \\
\text { test0017_va_exclude_12_tiles }\end{array}$ & Passed & 1.56 \\
\hline $7 / 62$ & $\begin{array}{l}\text { Test \#17: } \\
\text { Start 18: }\end{array}$ & $\begin{array}{l}\text { test0017_va_exclude_12_tiles } \ldots \ldots \ldots \ldots \ldots \ldots \\
\text { test0018_va_exclude_12_tiles_ini }\end{array}$ & Passed & 1.47 \\
\hline $8 / 62$ & $\begin{array}{l}\text { Test \#18: } \\
\text { Start 19: }\end{array}$ & $\begin{array}{l}\text { test0018_va_exclude_12_tiles_ini } \ldots \ldots \ldots \ldots \ldots \\
\text { test0019_va_exclude_12_rasters }\end{array}$ & Passe & 1.86 \\
\hline $19 / 62$ & $\begin{array}{l}\text { Test \#19: } \\
\text { Start } 20:\end{array}$ & $\begin{array}{l}\text { test0019_va_exclude_12_rasters } \ldots \ldots \ldots \ldots \ldots \ldots \\
\text { test0020_va_exclude_12_rasters_ini }\end{array}$ & Passed & 1.66 \\
\hline $20 / 62$ & $\begin{array}{l}\text { Test \#20: } \\
\text { Start 21: }\end{array}$ & $\begin{array}{l}\text { test0020_va_exclude_12_rasters_ini } \ldots \ldots \cdots \cdots \\
\text { test0021_va_exclude_1n_tiles }\end{array}$ & Passed & 1.77 \\
\hline $21 / 62$ & $\begin{array}{l}\text { Test \#21: } \\
\text { Start 22: }\end{array}$ & $\begin{array}{l}\text { test0021_va_exclude_1n_tiles } \ldots \cdots \cdots \cdots \cdots \cdots \\
\text { test0022_va_exclude_1n_tiles_ini }\end{array}$ & Passed & 1.64 \\
\hline $22 / 62$ & $\begin{array}{l}\text { Test \#22: } \\
\text { Start } 23:\end{array}$ & $\begin{array}{l}\text { test0022_va_exclude_1n_tiles_ini } \ldots \ldots \ldots \ldots \ldots \\
\text { test0023_va_exclude_1n_rasters }\end{array}$ & Passed & 1.91 \\
\hline $23 / 62$ & $\begin{array}{l}\text { Test \#23: } \\
\text { Start } 24:\end{array}$ & $\begin{array}{l}\text { test0023_va_exclude_1n_rasters } \ldots \ldots \cdots \cdots \cdots \\
\text { test0024_va_exclude_1n_rasters_ini }\end{array}$ & Passed & 1.84 \\
\hline $24 / 62$ & $\begin{array}{l}\text { Test \#24: } \\
\text { Start 25: }\end{array}$ & $\begin{array}{l}\text { test } 0024 \text { _va_exclude_1n_rasters_ini } \ldots \ldots \ldots \ldots \\
\text { test0025_va_exclude_2n_tiles }\end{array}$ & Passed & 1.69 \\
\hline $25 / 62$ & $\begin{array}{l}\text { Test \#25: } \\
\text { Start } 26:\end{array}$ & $\begin{array}{l}\text { test0025_va_exclude_2n_tiles } \ldots \cdots \cdots \cdots \cdots \cdots \\
\text { test0026_va_exclude_2n_tiles_ini }\end{array}$ & Passed & 1.34 \\
\hline $26 / 62$ & $\begin{array}{l}\text { Test \#26: } \\
\text { Start } 27:\end{array}$ & $\begin{array}{l}\text { est0026_va_exclude_2n_tiles_ini } \ldots \ldots \ldots \ldots \ldots \\
\text { est0027_va_exclude_2n_rasters }\end{array}$ & Passed & 1.37 \\
\hline $27 / 62$ & $\begin{array}{l}\text { Test \#27: } \\
\text { Start } 28:\end{array}$ & $\begin{array}{l}\text { test 0027_va_exclude_2n_rasters } \ldots \ldots \cdots \cdots \cdots \\
\text { test0028_va_exclude_2n_rasters_ini }\end{array}$ & Passed & 1.52 \\
\hline $8 / 62$ & Test \#28: & test0028_va_exclude_2n_rasters_ini $\ldots . . \ldots \ldots$ & Passed & 1.53 \\
\hline
\end{tabular}


Start 29: test0029_va_exclude_12n_tiles

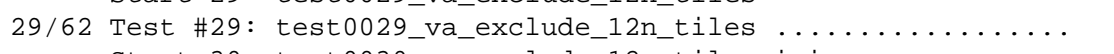
Start 30: test0030_va_exclude_12n_tiles_ini

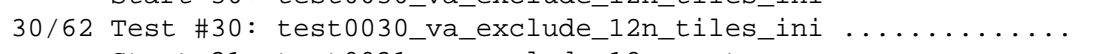
Start 31: test0031_va_exclude_12n_rasters

31/62 Test \#31: test0031_va_exclude_12n_rasters $\ldots \ldots \ldots \ldots \ldots \ldots$ Start 32: test0032_va_exclude_12n_rasters_ini

32/62 Test \#32: test0032_va_exclude_12n_rasters_ini ........... Start 33: test0033_i285_tiles

33/62 Test \#33: test0033_i285_tiles $\ldots \ldots \ldots \ldots \ldots \ldots \ldots \ldots \ldots \ldots$ Start 34: test0034_i285_rasters

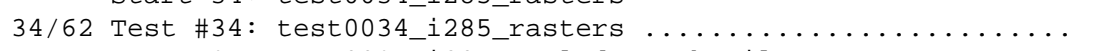
Start 35: test0035_i285_exclude_tech_tiles

35/62 Test \#35: test0035_i285_exclude_tech_tiles ............ Start 36: test0036_i285_exclude_tech_tiles_ini

36/62 Test \#36: test0036_i285_exclude_tech_tiles_ini .......... Start 37: test0037_i285_exclude_tech_rasters

37/62 Test \#37: test0037_i285_exclude_tech_rasters ............ Start 38: test0038_i285_exclude_tech_rasters_ini

38/62 Test \#38: test0038_i285_exclude_tech_rasters_ini ........ Start 39: test0039_i285_exclude_tech_fixed_tiles

39/62 Test \#39: test0039_i285_exclude_tech_fixed_tiles ........ Start 40: test0040_i285_exclude_tech_fixed_tiles_ini

40/62 Test \#40: test0040_i285_exclude_tech_fixed_tiles_ini ..... Start 41: test0041_i285_exclude_tech_fixed_rasters

41/62 Test \#41: test0041_i285_exclude_tech_fixed_rasters ...... Start 42: test0042_i285_exclude_tech_fixed_rasters_ini

42/62 Test \#42: test0042_i285_exclude_tech_fixed_rasters_ini... Start 43: test0043_mgrs

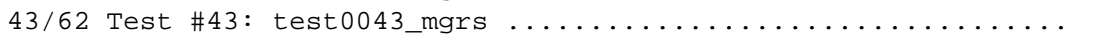
Start 44: test0044_va_exclude_12_mgrs_12_tiles

44/62 Test \#44: test0044_va_exclude_12_mgrs_12_tiles ......... Start 45: test0045_va_exclude_12_mgrs_12_tiles_ini

45/62 Test \#45: test0045_va_exclude_12_mgrs_12_tiles_ini ...... Start 46: test0046_va_exclude_12_mgrs_12_rasters

46/62 Test \#46: test0046_va_exclude_12_mgrs_12_rasters ........ Start 47: test0047_va_exclude_12_mgrs_12_rasters_ini

47/62 Test \#47: test0047_va_exclude_12_mgrs_12_rasters_ini .... Start 48: test0048_va_exclude_12_mgrs_1_tiles

48/62 Test \#48: test0048_va_exclude_12_mgrs_1_tiles .......... Start 49: test0049_va_exclude_12_mgrs_1_rasters

49/62 Test \#49: test0049_va_exclude_12_mgrs_1_rasters ......... Start 50: test0050_va_exclude_12_mgrs_2_tiles

50/62 Test \#50: test0050_va_exclude_12_mgrs_2_tiles .......... Start 51: test0051_va_exclude_12_mgrs_2_rasters

51/62 Test \#51: test0051_va_exclude_12_mgrs_2_rasters ......... Start 52: test0052_mosul_raster_only_inner

52/62 Test \#52: test0052_mosul_raster_only_inner ............. Start 53: test0053_mosul_raster_only_cut

53/62 Test \#53: test0053_mosul_raster_only_cut .............. Start 54: test0054_mosul_raster_only_outer

54/62 Test \#54: test0054_mosul_raster_only_outer ............. Start 55: test0055_mosul_raster_levant_raster_inner

55/62 Test \#55: test0055_mosul_raster_levant_raster_inner ..... Start 56: test0056_mosul_raster_levant_raster_cut

56/62 Test \#56: test0056_mosul_raster_levant_raster_cut ....... Start 57: test0057_mosul_raster_levant_raster_outer

57/62 Test \#57: test0057_mosul_raster_levant_raster_outer ..... Start 58: test0058_mosul_raster_levant_tile_inner

58/62 Test \#58: test0058_mosul_raster_levant_tile_inner ....... Start 59: test0059_mosul_raster_levant_tile_cut

59/62 Test \#59: test0059_mosul_raster_levant_tile_cut ......... Start 60: test0060_mosul_raster_levant_tile_outer

60/62 Test \#60: test0060_mosul_raster_levant_tile_outer ....... Start 61: test0061_mosul_rasters_only

\begin{tabular}{|c|c|c|}
\hline Passed & 1.41 & $\mathrm{sec}$ \\
\hline Passed & 1.41 & $\mathrm{sec}$ \\
\hline Passed & 1.55 & $\mathrm{sec}$ \\
\hline Passed & 1.55 & $\mathrm{sec}$ \\
\hline Passed & 0.73 & $\mathrm{sec}$ \\
\hline Passed & 0.69 & $\mathrm{sec}$ \\
\hline Passed & 0.78 & $\mathrm{sec}$ \\
\hline Passed & 0.81 & $\mathrm{sec}$ \\
\hline Passed & 0.77 & $\mathrm{sec}$ \\
\hline Passed & 0.77 & $\mathrm{sec}$ \\
\hline Passed & 0.81 & $\mathrm{sec}$ \\
\hline Passed & 0.80 & $\mathrm{sec}$ \\
\hline Passed & 0.73 & $\mathrm{sec}$ \\
\hline Passed & 0.75 & $\mathrm{sec}$ \\
\hline Passed & 0.06 & $\mathrm{sec}$ \\
\hline Passed & 1.45 & $\mathrm{sec}$ \\
\hline Passed & 1.36 & $\mathrm{sec}$ \\
\hline Passed & 1.50 & $\mathrm{sec}$ \\
\hline Passed & 1.52 & $\mathrm{sec}$ \\
\hline Passed & 1.34 & $\mathrm{sec}$ \\
\hline Passed & 1.55 & $\mathrm{sec}$ \\
\hline Passed & 1.41 & $\mathrm{sec}$ \\
\hline Passed & 1.84 & $\mathrm{sec}$ \\
\hline Passed & 0.84 & $\mathrm{sec}$ \\
\hline Passed & 1.30 & $\mathrm{sec}$ \\
\hline Passed & 1.17 & $\mathrm{sec}$ \\
\hline Passed & 0.67 & $\mathrm{sec}$ \\
\hline Passed & 1.50 & $\mathrm{sec}$ \\
\hline Passed & 1.42 & $\mathrm{sec}$ \\
\hline Passed & 0.81 & $\mathrm{sec}$ \\
\hline Passed & 1.59 & \\
\hline Passed & 1.23 & \\
\hline
\end{tabular}

Passed $1.23 \mathrm{sec}$ 


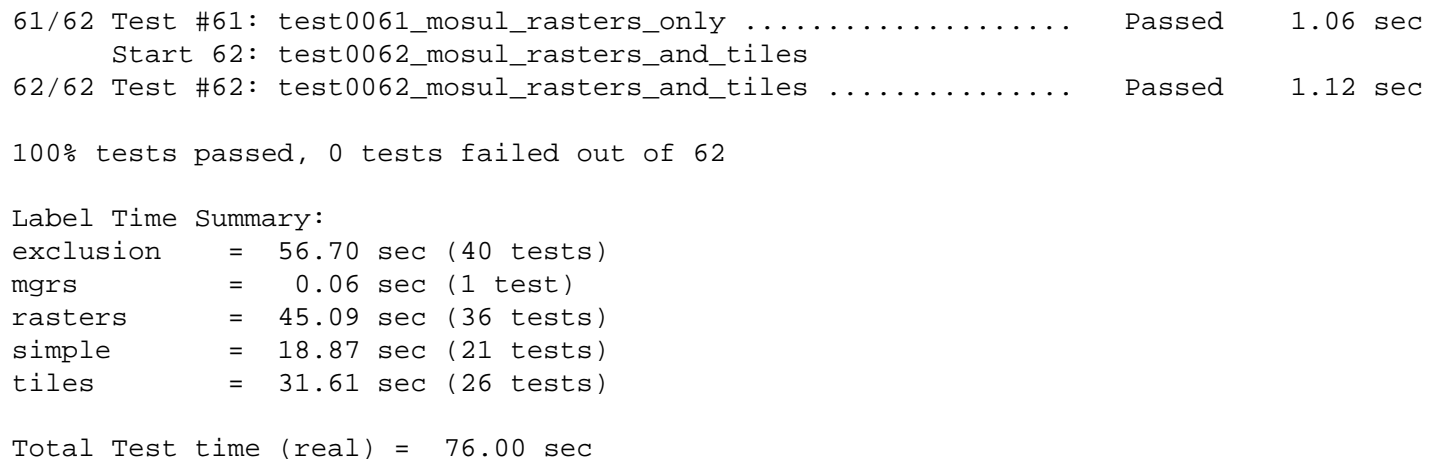




\subsection{AUTOMATED TEST COVERAGE}

Test coverage analysis is produced using version 0.7 .9 of JaCoco. ${ }^{2}$ Just as not all NFAC components have equal importance in providing functionality, not all branches within any NFAC component have equal importance. Coverage results from the JaCoCo report are presented in the following tables. Table 3 gives a summary by Java package..$^{\dagger}$

Table 3: Code Coverage by Package

\begin{tabular}{|c|c|c|c|c|c|c|c|c|c|c|}
\hline Package & $\begin{array}{r}\text { Instructions } \\
\text { Coverage (\%) }\end{array}$ & $\begin{array}{r}\text { Branches } \\
\text { Coverage (\%) }\end{array}$ & $\begin{array}{r}\text { Complexity } \\
\text { Missed }\end{array}$ & $\begin{array}{r}\text { Complexity } \\
\text { Total }\end{array}$ & $\begin{array}{r}\text { Lines } \\
\text { Missed }\end{array}$ & $\begin{array}{r}\text { Lines } \\
\text { Total }\end{array}$ & $\begin{array}{r}\text { Mehods } \\
\text { Missed }\end{array}$ & $\begin{array}{r}\text { Methods } \\
\text { Total } \\
\end{array}$ & $\begin{array}{r}\text { Classes } \\
\text { Missed }\end{array}$ & $\begin{array}{r}\text { Classes } \\
\text { Total }\end{array}$ \\
\hline CAcomp & 92 & 59 & 14 & 41 & 2 & 64 & 2 & 25 & 0 & 1 \\
\hline CAcomp.data & 56 & 35 & 1,040 & 1,593 & 1,494 & 3,510 & 373 & 785 & 5 & 39 \\
\hline CAcomp.data.analyst & 47 & 30 & 796 & 1,242 & 1,575 & 2,978 & 218 & 525 & 4 & 26 \\
\hline CAcomp.data.analyst.plantcond & 42 & 7 & 237 & 389 & 534 & 983 & 130 & 278 & 0 & 14 \\
\hline CAcomp.data.times & 91 & 61 & 35 & 104 & 22 & 234 & 5 & 61 & 0 & 13 \\
\hline CAcomp.data.impl & 72 & 47 & 668 & 1,045 & 1,034 & 3,578 & 83 & 227 & 2 & 14 \\
\hline CAcomp.exception & 0 & $\mathrm{n} / \mathrm{a}$ & 3 & 3 & 6 & 6 & 3 & 3 & 1 & 1 \\
\hline
\end{tabular}

Coverage for each class is presented in Tables 4-10. In terms of significance in NFAC processing, the most important classes are:

- CAcomp.data.AmbClient

- CAcomp.data.Facility

- CAcomp.data.ModelTimes

- CAcomp.data.Nfaclncident

- CAcomp.data.NfaclncidentMgr

- CAcomp.data.ParticleGroups

- CAcomp.data.ParticleGroupsMgr

- CAcomp.data.analyst.PercentInventory

- CAcomp.data.analyst.PercentInventoryRelease

- CAcomp.data.analyst.PercentInventoryReleaseTree

- CAcomp.data.analyst.SourceTermTable

- all classes in CAcomp.data.times

- CAcomp.impl.AmblnputsMgr

- CAcomp.impl.Nfaclmpl

- CAcomp.impl.ReproMod

- CAcomp.impl.St_Monitor

- CAcomp.impl.StcalcClient

\footnotetext{
${ }^{\dagger}$ Package names assume a mil.dtra.hpac.model.nfac prefix.
} 
Table 4: Code Coverage: CAComp

\begin{tabular}{|l|rr|rr|rr|rr|}
\hline Class & $\begin{array}{r}\text { Instructions } \\
\text { Coverage (\%) }\end{array}$ & $\begin{array}{r}\text { Branches } \\
\text { Coverage (\%) }\end{array}$ & $\begin{array}{r}\text { Complexity } \\
\text { Missed }\end{array}$ & $\begin{array}{r}\text { Complexity } \\
\text { Total }\end{array}$ & $\begin{array}{r}\text { Lines } \\
\text { Missed }\end{array}$ & $\begin{array}{r}\text { Lines } \\
\text { Total }\end{array}$ & $\begin{array}{r}\text { Methods } \\
\text { Missed }\end{array}$ & $\begin{array}{r}\text { Methods } \\
\text { Total }\end{array}$ \\
\hline \hline NfacServiceRefs & 92 & 59 & 14 & 41 & 2 & 64 & 2 & 25 \\
\hline
\end{tabular}

Table 5: Code Coverage: CAComp.data

\begin{tabular}{|c|c|c|c|c|c|c|c|c|}
\hline Class & $\begin{array}{r}\text { Instructions } \\
\text { Coverage (\%) }\end{array}$ & $\begin{array}{r}\text { Branches } \\
\text { Coverage }(\%)\end{array}$ & $\begin{array}{r}\text { Complexity } \\
\text { Missed }\end{array}$ & $\begin{array}{r}\text { Complexity } \\
\text { Total }\end{array}$ & $\begin{array}{l}\text { Lines } \\
\text { Missed }\end{array}$ & $\begin{array}{r}\text { Lines } \\
\text { Total }\end{array}$ & $\begin{array}{r}\text { Methods } \\
\text { Missed }\end{array}$ & $\begin{array}{r}\text { Methods } \\
\text { Total }\end{array}$ \\
\hline ActivityHash & $\begin{array}{ll} \\
\end{array}$ & $\begin{array}{ll} \\
\end{array}$ & 28 & $\overline{28}$ & 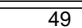 & $\overline{499}$ & $\overline{12}$ & 1212 \\
\hline ActivityHash.ActivityZComparator & 0 & 0 & 7 & 7 & 6 & 6 & 4 & 4 \\
\hline AmbClient & 66 & 46 & 133 & 223 & 154 & 533 & 15 & 74 \\
\hline AmbClient.ActivityNameSorter & 51 & 0 & 3 & 5 & 2 & 6 & 2 & 4 \\
\hline AmbClient.Activity TableInputMatIDSorter & 46 & 25 & 6 & 8 & 2 & 7 & 2 & 4 \\
\hline AmbClient.Activity ValueSorter & 0 & 0 & 5 & 5 & 4 & 4 & 4 & 4 \\
\hline AmbClient.IsotopeDepSorter & 50 & 37 & 9 & 12 & 2 & 11 & 2 & 4 \\
\hline AmbClient.IsotopeShortNameSorter & 37 & 25 & 6 & 8 & 2 & 5 & 2 & 4 \\
\hline AmbClient.Refs & 100 & $\mathrm{n} / \mathrm{a}$ & 0 & 1 & 0 & 6 & 0 & 1 \\
\hline AmbClient.ReleaseMCSorter & 71 & 50 & 10 & 14 & 4 & 19 & 2 & 4 \\
\hline CountryCodeMap & 54 & 42 & 8 & 16 & 18 & 48 & 3 & 9 \\
\hline DummylnstanceValue & 0 & $n / a$ & 6 & 6 & 7 & 7 & 6 & 6 \\
\hline Element & 11 & 9 & 37 & 44 & 79 & 100 & 17 & 23 \\
\hline Facility & 66 & 28 & 92 & 158 & 97 & 330 & 12 & 68 \\
\hline FacilityDB & 80 & 50 & 6 & 13 & 4 & 22 & 3 & 9 \\
\hline FacilityDBMgr & 54 & 40 & 9 & 18 & 25 & 69 & 2 & 8 \\
\hline FacilityDef & 71 & 47 & 54 & 107 & 57 & 238 & 8 & 44 \\
\hline Fastlsotopes & 35 & 31 & 22 & 34 & 35 & 67 & 8 & 15 \\
\hline MaterialMode & 92 & 100 & 1 & 7 & 0 & 7 & 1 & 5 \\
\hline ModelDefUtils & 35 & 23 & 87 & 98 & 133 & 206 & 18 & 27 \\
\hline ModelTimes & 78 & 26 & 27 & 68 & 32 & 186 & 4 & 45 \\
\hline ModerateModel & 35 & 0 & 8 & 15 & 7 & 16 & 5 & 12 \\
\hline NfacConstants & 100 & $\mathrm{n} / \mathrm{a}$ & 0 & 1 & 0 & 2 & 0 & 1 \\
\hline NfacDummyMaterial & 0 & 0 & 136 & 136 & 162 & 162 & 131 & 131 \\
\hline Nfaclncident & 66 & 13 & 22 & 49 & 47 & 133 & 6 & 31 \\
\hline NfaclncidentMgr & 48 & 47 & 35 & 63 & 103 & 196 & 19 & 39 \\
\hline NfacIncidentMgr.MCParameters & 100 & $\mathrm{n} / \mathrm{a}$ & 0 & 1 & 0 & 4 & 0 & 1 \\
\hline NfacRelease & 89 & 50 & 7 & 20 & 5 & 38 & 3 & 16 \\
\hline Options & 47 & 10 & 31 & 63 & 67 & 175 & 8 & 39 \\
\hline ParticleGroups & 72 & 48 & 59 & 95 & 74 & 273 & 8 & 24 \\
\hline ParticleGroups.GroupActivityList & 81 & 62 & 10 & 22 & 7 & 47 & 3 & 6 \\
\hline ParticleGroups.GroupInfo & 64 & 50 & 6 & 13 & 5 & 18 & 1 & 8 \\
\hline ParticleGroups.Melcorlnfo & 41 & 40 & 13 & 19 & 8 & 21 & 3 & 9 \\
\hline ParticleGroupsMgr & 71 & 47 & 19 & 40 & 21 & 88 & 3 & 16 \\
\hline PropertyFinder & 87 & 73 & 16 & 38 & 15 & 100 & 0 & 8 \\
\hline Range & 19 & 15 & 40 & 45 & 69 & 86 & 13 & 16 \\
\hline Range.Mode & 76 & $\mathrm{n} / \mathrm{a}$ & 2 & 4 & 0 & 3 & 2 & 4 \\
\hline Reprolnventory & 11 & 11 & 70 & 74 & 183 & 206 & 34 & 38 \\
\hline SevereModel & 29 & 0 & 10 & 15 & 9 & 16 & 7 & 12 \\
\hline
\end{tabular}

Table 6: Code Coverage: CAComp.data.analyst

\begin{tabular}{|c|c|c|c|c|c|c|c|c|}
\hline Class & $\begin{array}{r}\text { Instructions } \\
\text { Coverage (\%) }\end{array}$ & $\begin{array}{r}\text { Branches } \\
\text { Coverage (\%) } \\
\end{array}$ & $\begin{array}{r}\text { Complexity } \\
\text { Missed }\end{array}$ & $\begin{array}{r}\text { Complexity } \\
\text { Total }\end{array}$ & $\begin{array}{r}\text { Lines } \\
\text { Missed }\end{array}$ & $\begin{array}{l}\text { Lines } \\
\text { Total }\end{array}$ & $\begin{array}{r}\text { Methods } \\
\text { Missed }\end{array}$ & $\begin{array}{r}\text { Methods } \\
\text { Total }\end{array}$ \\
\hline AbstractAnalystModel & 63 & $\mathrm{n} / \mathrm{a}$ & 1 & 6 & 3 & 8 & 1 & 6 \\
\hline AnalystMix & 55 & 25 & 27 & 49 & 60 & 126 & 12 & 31 \\
\hline AnalystModelUtils & 62 & 62 & 7 & 9 & 4 & 11 & 4 & 5 \\
\hline ConcentrationUnits & 30 & 0 & 21 & 32 & 50 & 76 & 12 & 23 \\
\hline ContainmentMonitorReading & 48 & 10 & 24 & 44 & 63 & 135 & 9 & 29 \\
\hline IsotopeReleases & 33 & 20 & 42 & 58 & 81 & 120 & 18 & 31 \\
\hline IsotopeValue & 27 & 12 & 41 & 55 & 87 & 123 & 18 & 30 \\
\hline IsotopicConcentrations & 46 & 10 & 25 & 42 & 65 & 124 & 11 & 27 \\
\hline IsotopicReleaseRates & 48 & 14 & 22 & 38 & 51 & 104 & 9 & 24 \\
\hline PIRSubReleaseMode & 92 & 100 & 1 & 7 & 0 & 7 & 1 & 5 \\
\hline PercentInventory & 56 & 44 & 46 & 83 & 83 & 215 & 9 & 28 \\
\hline PercentInventoryConstants & 0 & $\mathrm{n} / \mathrm{a}$ & 1 & 1 & 2 & 2 & 1 & 1 \\
\hline PercentInventoryRelease & 51 & 31 & 37 & 69 & 76 & 154 & 16 & 42 \\
\hline PercentInventoryReleaseTree & 91 & 62 & 84 & 150 & 32 & 361 & 6 & 37 \\
\hline PlantConditionConstants & 55 & 13 & 31 & 35 & 51 & 60 & 8 & 12 \\
\hline PlantConditionTables & 31 & 38 & 62 & 102 & 156 & 230 & 11 & 24 \\
\hline PlantConditionsModel & 100 & $\mathrm{n} / \mathrm{a}$ & 0 & 1 & 0 & 1 & 0 & 1 \\
\hline RascalFileReader & 0 & 0 & 89 & 89 & 233 & 233 & 15 & 15 \\
\hline RascalProject & 40 & 22 & 62 & 90 & 134 & 228 & 14 & 35 \\
\hline RascalXmIReader & 0 & 0 & 51 & 51 & 117 & 117 & 9 & 9 \\
\hline RascalXmIReader.ReleaseStepRec & 0 & $\mathrm{n} / \mathrm{a}$ & 1 & 1 & 5 & 5 & 1 & 1 \\
\hline ReleaseRate & 31 & 0 & 22 & 35 & 57 & 90 & 12 & 25 \\
\hline ReleaseUnits & 44 & 5 & 17 & 32 & 40 & 76 & 8 & 23 \\
\hline SourceTermTable & 73 & 55 & 53 & 109 & 53 & 209 & 3 & 26 \\
\hline SourceTermTable.Operationallnfo & 94 & $n / a$ & 1 & 3 & 2 & 14 & 1 & 3 \\
\hline SpentFuel & 48 & 13 & 28 & 51 & 70 & 149 & 9 & 32 \\
\hline
\end{tabular}


Table 7: Code Coverage: CAComp.data.analyst.plantcond

\begin{tabular}{|c|c|c|c|c|c|c|c|c|}
\hline Class & $\begin{array}{r}\text { Instructions } \\
\text { Coverage (\%) } \\
\end{array}$ & $\begin{array}{r}\text { Branches } \\
\text { Coverage }(\%) \\
\end{array}$ & $\begin{array}{r}\text { Complexity } \\
\text { Missed }\end{array}$ & $\begin{array}{r}\text { Complexity } \\
\text { Total } \\
\end{array}$ & $\begin{array}{r}\text { Lines } \\
\text { Missed }\end{array}$ & $\begin{array}{r}\text { Lines } \\
\text { Total }\end{array}$ & $\begin{array}{r}\text { Methods } \\
\text { Missed }\end{array}$ & $\begin{array}{r}\text { Methods } \\
\text { Total } \\
\end{array}$ \\
\hline PCConfinementByPass & 29 & 0 & 10 & 15 & 13 & 22 & 7 & 12 \\
\hline PCConfinementLeakage & 29 & 0 & 10 & 15 & 13 & 22 & 7 & 12 \\
\hline PCContainmentByPass & 37 & 0 & 17 & 26 & 39 & 62 & 11 & 20 \\
\hline PCContainmentLeakage & 55 & $\mathrm{n} / \mathrm{a}$ & 5 & 9 & 8 & 18 & 5 & 9 \\
\hline PCCoreLeakData & 51 & 33 & 15 & 30 & 39 & 82 & 7 & 18 \\
\hline PCDryWellLeakage & 42 & 0 & 17 & 28 & 40 & 73 & 10 & 21 \\
\hline PClceCondenserContainment & 45 & 0 & 19 & 34 & 48 & 100 & 10 & 25 \\
\hline PCLargeDrySubContainmentLeakage & 42 & 0 & 17 & 28 & 40 & 74 & 10 & 21 \\
\hline PCPromptCriticalPowerExcursion & 42 & 9 & 22 & 34 & 43 & 76 & 11 & 23 \\
\hline PCReproFacility & 42 & 0 & 22 & 44 & 76 & 155 & 10 & 32 \\
\hline PCSodiumWaterReaction & 24 & 0 & 14 & 19 & 20 & 29 & 9 & 14 \\
\hline PCSteamTubeRupture & 42 & 11 & 30 & 47 & 65 & 119 & 12 & 29 \\
\hline PCSubatmosphericConfinementLeakage & 36 & 0 & 16 & 25 & 40 & 66 & 10 & 19 \\
\hline PCWetWellLeakage & 40 & 8 & 23 & 35 & 50 & 85 & 11 & 23 \\
\hline
\end{tabular}

Table 8: Code Coverage: CAComp.data.times

\begin{tabular}{|c|c|c|c|c|c|c|c|c|}
\hline Class & $\begin{array}{r}\text { Instructions } \\
\text { Coverage (\%) } \\
\end{array}$ & $\begin{array}{r}\text { Branches } \\
\text { Coverage (\%) } \\
\end{array}$ & $\begin{array}{r}\text { Complexity } \\
\text { Missed }\end{array}$ & $\begin{array}{r}\text { Complexity } \\
\text { Total } \\
\end{array}$ & $\begin{array}{r}\text { Lines } \\
\text { Missed }\end{array}$ & $\begin{array}{r}\text { Lines } \\
\text { Total } \\
\end{array}$ & $\begin{array}{r}\text { Methods } \\
\text { Missed } \\
\end{array}$ & $\begin{array}{r}\text { Methods } \\
\text { Total } \\
\end{array}$ \\
\hline AbstractTimesMgr & 90 & 50 & 6 & 12 & 2 & 19 & 2 & 8 \\
\hline AnalystMixTimesMgr & 100 & $n / a$ & 0 & 4 & 0 & 6 & 0 & 4 \\
\hline IsotopicConcentrationsTimesMgr & 100 & $n / a$ & 0 & 4 & 0 & 7 & 0 & 4 \\
\hline IsotopicReleaseRatesTimesMgr & 100 & $\mathrm{n} / \mathrm{a}$ & 0 & 4 & 0 & 8 & 0 & 4 \\
\hline LeakRateTimesMgr & 89 & 50 & 4 & 9 & 2 & 27 & 0 & 5 \\
\hline ModelTimesMgrFactory & 68 & 70 & 6 & 15 & 4 & 12 & 1 & 3 \\
\hline NoopTimesMgr & 83 & $n / a$ & 2 & 4 & 2 & 8 & 2 & 4 \\
\hline OperationalTimesMgr & 81 & 45 & 10 & 14 & 8 & 42 & 0 & 4 \\
\hline PCContainmentByPassTimesMgr & 100 & $n / a$ & 0 & 4 & 0 & 7 & 0 & 4 \\
\hline PCLeakRateTimesMgr & 100 & $n / a$ & 0 & 1 & 0 & 2 & 0 & 1 \\
\hline PercentInventoryTimesMgr & 100 & 83 & 3 & 19 & 0 & 59 & 0 & 10 \\
\hline RascalProjectTimesMgr & 91 & 50 & 4 & 10 & 4 & 29 & 0 & 6 \\
\hline SpentFuelTimesMgr & 100 & $\mathrm{n} / \mathrm{a}$ & 0 & 4 & 0 & 8 & 0 & 4 \\
\hline
\end{tabular}

Table 9: Code Coverage: CAComp.exceptions

\begin{tabular}{|l|rr|rr|r|r|r|r|}
\hline Class & $\begin{array}{r}\text { Instructions } \\
\text { Coverage (\%) }\end{array}$ & $\begin{array}{r}\text { Branches } \\
\text { Coverage (\%) }\end{array}$ & $\begin{array}{r}\text { Complexity } \\
\text { Missed }\end{array}$ & $\begin{array}{r}\text { Complexity } \\
\text { Total }\end{array}$ & $\begin{array}{r}\text { Lines } \\
\text { Missed }\end{array}$ & $\begin{array}{r}\text { Lines } \\
\text { Total }\end{array}$ & $\begin{array}{r}\text { Methods } \\
\text { Missed }\end{array}$ & $\begin{array}{r}\text { Methods } \\
\text { Total }\end{array}$ \\
\hline \hline NfacValidationException & 0 & $\mathrm{n} / \mathrm{a}$ & 3 & 3 & 6 & 6 & 3 & 3 \\
\hline
\end{tabular}

Table 10: Code Coverage: CAComp.impl

\begin{tabular}{|c|c|c|c|c|c|c|c|c|}
\hline Class & $\begin{array}{r}\text { Instructions } \\
\text { Coverage (\%) } \\
\end{array}$ & $\begin{array}{r}\text { Branches } \\
\text { Coverage }(\%) \\
\end{array}$ & $\begin{array}{r}\text { Complexity } \\
\text { Missed }\end{array}$ & $\begin{array}{r}\text { Complexity } \\
\text { Total }\end{array}$ & $\begin{array}{r}\text { Lines } \\
\text { Missed }\end{array}$ & $\begin{array}{l}\text { Lines } \\
\text { Total }\end{array}$ & $\begin{array}{r}\text { Methods } \\
\text { Missed }\end{array}$ & $\begin{array}{r}\text { Methods } \\
\text { Total } \\
\end{array}$ \\
\hline AmblnputsMgr & 84 & 58 & 77 & 141 & 56 & 430 & 8 & 35 \\
\hline AmblnputsMgr.MCReleaseRec & 92 & 62 & 6 & 11 & 2 & 30 & 0 & 3 \\
\hline EfficiencyFile & 92 & 70 & 4 & 12 & 1 & 32 & 1 & 7 \\
\hline EquilibrateActivityVector & 0 & 0 & 36 & 36 & 115 & 115 & 12 & 12 \\
\hline MelcorFraction & 38 & 14 & 15 & 20 & 13 & 25 & 8 & 13 \\
\hline MelcorFractions & 83 & 53 & 12 & 19 & 2 & 37 & 1 & 5 \\
\hline MelcorReleaseFractionsFile & 82 & 83 & 2 & 7 & 2 & 18 & 1 & 4 \\
\hline NfacFieldDescribeHelper & 0 & 0 & 155 & 155 & 405 & 405 & 35 & 35 \\
\hline Nfaclmpl & 69 & 48 & 91 & 145 & 158 & 589 & 9 & 45 \\
\hline PercentInventoryFileMgr & 66 & 45 & 8 & 13 & 6 & 25 & 0 & 3 \\
\hline RepoData & 58 & 43 & 14 & 26 & 22 & 58 & 3 & 10 \\
\hline ReproMod & 100 & 100 & 0 & 58 & 0 & 413 & 0 & 13 \\
\hline St_Monitor & 90 & 42 & 8 & 12 & 6 & 23 & 1 & 5 \\
\hline StcalcClient & 81 & 55 & 240 & 390 & 246 & 1,378 & 4 & 37 \\
\hline
\end{tabular}




\subsection{INTERACTIVE TEST RESULTS}

Table 11: Interactive Test Results: Top Level Interactions

\begin{tabular}{ll}
\hline \hline Test & Result \\
Set Incident Name & Success
\end{tabular}

Table 12: Interactive Test Results: Where Tab

\begin{tabular}{ll}
\hline \hline Test & Result \\
Select facility & Success \\
Select default facility location & Success \\
Enter explicit facility location & Success \\
Define facility location by dragging the incident Icon & Success \\
Select the default inventory file & Success \\
Select a custom inventory file & Success \\
Use the ORIGEN File Import Utility to create a custom inventory file & Success
\end{tabular}


Table 13: Interactive Test Results: What Tab

\begin{tabular}{ll}
\hline \hline Test & Result \\
Enter an explicit operating power & Success \\
Select a moderate incident & Success \\
Select a severe incident & Success \\
Define an Isotopic Release Rates incident & Success \\
Define an Isotopic Concentrations incident & Success \\
Define a Percent Inventory incident & Success \\
Define an Analyst Mix incident & Success \\
Define a Containment Monitor Reading incident & Success \\
Define a Spent Fuel incident & Sucess \\
Define a RASCAL Project incident & Success \\
Define a Confinement Bypass Plant Conditions incident & Success \\
Define a Confinement Leakage/Failure Plant Conditions incident & Success \\
Define a Containment Bypass Plant Conditions incident & Success \\
Define a Containment Leakage/Failure Plant Conditions incident & Success \\
Define a Dry Well Leakage/Failure Plant Conditions incident & Success \\
Define an Ice Condenser Containment Leakage/Failure Plant Conditions incident & Success \\
Define a Large Dry Subcontainmet Leakage/Failure Plant Conditions incident & Success \\
Define a Prompt Critical Power Excursion Plant Condition incident & Success \\
Define a Reprocessing Facility Plant Condition incident & Success \\
Define a Steam Generator Tube Rupture Plant Condition incident & Success \\
Define a Subatmospheric Confinement Leakage/Failure Plant Conditions incident & Success \\
Define a Wet Well Leakage/Failure Plant Conditions incident & Success \\
Select Material Processing Modes & Success \\
Select Percent Inventory Release Modes & Success \\
View source term & Success
\end{tabular}


Table 14: Interactive Test Results: When Tab

\begin{tabular}{lc}
\hline \hline Test & Result \\
Moderate model times edit & Success \\
Severe model times edit & Success \\
Analyst Mix model times edit & Success \\
Containment Monitor Reading model times edit & Success \\
Isotopic Concentrations model times edit & Success \\
Isotopic Release Rates model times edit & Success \\
Percent Inventory model times edit & Success \\
Containment Bypass Plant Conditions model Times edit & Success \\
Prompt Critical Power Excursion Plant Conditions model times edit & Success \\
Reprocessing Facility Plant Conditions model times edit & Success \\
Confinement Bypass Plant Conditions model times edit & Success \\
Spent Fuel model times edit & Success \\
RASCAL Project model times edit & Success \\
Detect event times out of chronological order & Success \\
Apply time field changes for all events & Success \\
Override times in advanced mode & Success
\end{tabular}

Table 15: Interactive Test Results: Notes Tab

\begin{tabular}{ll}
\hline Test & Result \\
Edit notes & Success
\end{tabular}




\section{References}

1. E.R. Buckle. Nucleation and growth of caesium iodide aerosols. J. Aerosol Sci., 22(2):135-147, 1991.

2. JaCoCo: Java Code Coverage Library. http://www.eclemma.org/jacoco/.

3. R.W. Lee, R.W. Morris, and C.D. Sulfredge. Nuclear facility accident (nfac) unit test report for hpac version 6.3. Technical Report ORNL/TM-2015/726, Oak Ridge National Laboratory, December 2015.

4. State-of-the-art reactor consequence analysis project, volume 1:peach bottom integrated analysis. Technical Report NUREG/CR-7110, Vol. 1, Rev. 1, Sandia National Laboratory, May 2013.

5. State-of-the-art reactor consequence analysis project, volume 2:surry integrated analysis. Technical Report NUREG/CR-7110, Vol. 2, Rev. 1, Sandia National Laboratory, August 2013.

6. State-of-the-art reactor consequence analysis project, uncertainty analysis of the unmitigated long-term station blackout of the peach bottom atomic power station. Technical Report NUREG/CR-7155, Sandia National Laboratory, May 2016. 
ORNL/TM-2017/94

\section{INTERNAL DISTRIBUTION}

1. R. W. Lee

2. C. D. Sulfredge

3. ORNL Office of Technical Information and Classification

\section{EXTERNAL DISTRIBUTION}

4. Todd Hann, Chief, Hazards \& Effects Modeling Division, Information Sciences \& Applications Department, Defense Threat Reduction Agency, 8725 John J. Kingman Rd., Stop 6201, Ft Belvoir, VA 22060-6201 